\title{
The habitability of Proxima Centauri b
}

\section{Possible climates and observability}

\author{
Martin Turbet ${ }^{1}$, Jérémy Leconte ${ }^{2}$, Franck Selsis ${ }^{2}$, Emeline Bolmont $^{3}$, François Forget ${ }^{1}$, Ignasi Ribas ${ }^{4}$, \\ Sean N. Raymond ${ }^{2}$, and Guillem Anglada-Escudé ${ }^{5}$ \\ ${ }^{1}$ Laboratoire de Météorologie Dynamique, Sorbonne Universités, UPMC Univ. Paris 06, CNRS, 4 place Jussieu, 75005 Paris, France \\ e-mail: mturbet@lmd.jussieu.fr \\ ${ }^{2}$ Laboratoire d'astrophysique de Bordeaux, Univ. Bordeaux, CNRS, B18N, Allée Geoffroy Saint-Hilaire, 33615 Pessac, France \\ 3 NaXys, Department of Mathematics, University of Namur, 8 rempart de la Vierge, 5000 Namur, Belgium \\ ${ }^{4}$ Institut de Ciències de l'Espai (IEEC-CSIC), C/Can Magrans, s/n, Campus UAB, 08193 Bellaterra, Spain \\ 5 School of Physics and Astronomy, Queen Mary University of London, 327 Mile End Rd, London E1 4NS, UK
}

Received 24 August 2016 / Accepted 27 September 2016

\begin{abstract}
Radial velocity monitoring has found the signature of a $M \sin i=1.3 M_{\oplus}$ planet located within the habitable zone (HZ) of Proxima Centauri. Despite a hotter past and an active host star, the planet Proxima $b$ could have retained enough volatiles to sustain surface habitability. Here we use a 3D Global Climate Model (GCM) to simulate the atmosphere and water cycle of Proxima b for its two likely rotation modes (1:1 and 3:2 spin-orbit resonances), while varying the unconstrained surface water inventory and atmospheric greenhouse effect. Any low-obliquity, low-eccentricity planet within the HZ of its star should be in one of the climate regimes discussed here. We find that a broad range of atmospheric compositions allow surface liquid water. On a tidally locked planet with sufficient surface water inventory, liquid water is always present, at least in the substellar region. With a non-synchronous rotation, this requires a minimum greenhouse warming $\left(\sim 10 \mathrm{mbar}\right.$ of $\mathrm{CO}_{2}$ and 1 bar of $\left.\mathrm{N}_{2}\right)$. If the planet is dryer, $\sim 0.5$ bar or 1.5 bars of $\mathrm{CO}_{2}$ (for asynchronous or synchronous rotation, respectively) suffice to prevent the trapping of any arbitrary, small water inventory into polar or nightside ice caps. We produce reflection and emission spectra and phase curves for the simulated climates. We find that atmospheric characterization will be possible via direct imaging with forthcoming large telescopes. The angular separation of $7 \lambda / D$ at $1 \mu \mathrm{m}$ (with the E-ELT) and a contrast of $\sim 10^{-7}$ will enable high-resolution spectroscopy and the search for molecular signatures, including $\mathrm{H}_{2} \mathrm{O}, \mathrm{O}_{2}$, and $\mathrm{CO}_{2}$. The observation of thermal phase curves can be attempted with the James Webb Space Telescope, thanks to a contrast of $2 \times 10^{-5}$ at $10 \mu \mathrm{m}$. Proxima b will also be an exceptional target for future IR interferometers. Within a decade it will be possible to image Proxima $b$ and possibly determine whether the surface of this exoplanet is habitable.
\end{abstract}

Key words. stars: individual: Proxima Cen - planets and satellites: individual: Proxima Cen b - planets and satellites: atmospheres planets and satellites: terrestrial planets - planets and satellites: detection - astrobiology

\section{Introduction}

Proxima Centauri b, recently discovered by Anglada-Escudé et al. (2016), is not only the closest known extrasolar planet but also the closest potentially habitable terrestrial world, located at only 4.2 light years from the Earth (Van Leeuwen 2007).

Proxima Centauri b, also called Proxima b, receives a stellar flux of $\sim 950 \mathrm{~W} \mathrm{~m}^{-2}\left(0.65-0.7 S_{\oplus}\right.$ at $0.05 \mathrm{AU}$ based on the bolometric luminosity from Demory et al. 2009; Boyajian et al. 2012); this places Proxima b undoubtedly well within the socalled habitable zone $(\mathrm{HZ})$ of its host star $\left(M_{\star}=0.123 M_{\odot}\right)$, which is defined as the range of orbital distances within which a planet can possibly maintain liquid water on its surface (Kasting et al. 1993; Kopparapu et al. 2013, 2014, 2016; Leconte et al. 2013a; Yang et al. 2013). Indeed, for the effective temperature of Proxima (3050 K; Anglada-Escudé et al. 2016) climate models locate the inner edge between 0.9 and $1.5 S_{\oplus}$ depending on the planet rotation (Kopparapu et al. 2016), and the outer edge at $\sim 0.2 S_{\oplus}$ (Kopparapu et al. 2013). Nonetheless, surface habitability requires the planet to be endowed with a sufficient amount of water and atmospheric gases able to maintain a surface pressure and possibly a greenhouse effect (typically with $\mathrm{CO}_{2}$ ).

Quantifying this last statement is the main goal of this study. While most previous studies on climate and habitability focused on estimating the edges of the habitable zone, here we rather investigate the variety of necessary atmospheric compositions and global water content to ensure surface liquid water. Using the limited amount of information available on Proxima b, we can already provide some constraints on its possible climate regimes as a function of a key parameter: the volatile inventory, which includes the amount of available water above the surface and the amount and type of greenhouse and background gases in the atmosphere. Investigating extreme inventory scenarios is especially important in our specific case because Proxima is an active M dwarf. This means that the atmospheric content of the planet has probably been dramatically influenced by various types of escape, especially during the pre-main-sequence phase in which the planet underwent a runaway greenhouse phase. See the companion paper by Ribas et al. (2016) for a detailed discussion. 
Assuming a circular orbit, Proxima b should be in synchronous rotation with permanent dayside and nightside (1:1 resonance). However, Ribas et al. (2016) showed that the orbit of Proxima $b$ might not have had time to circularize and that an eccentricity above $\sim 0.06$ would be sufficient to capture the planet into a 3:2 spin-orbit resonance similar to Mercury. At higher eccentricities, higher resonances such as the 2:1 become possible as well. The climate on a tidally locked (synchronous) planet can dramatically differ from the asynchronous case. For a given volatile inventory, we will thus systematically try to infer the difference in behavior between a planet in a $1: 1$ and $3: 2$ resonance. The choice of the specific resonance order, however, has a much more subtle impact on the climate, so that the investigations of higher order resonances will be left for further studies.

Guided by various works on previously observed terrestrial exoplanets (Wordsworth et al. 2011; Pierrehumbert 2011; Leconte et al. 2013b), this study thus explores the climate regimes available for Proxima $b$ as a function of its spin state, atmospheric composition and thickness, and total amount of water available in the system. For this purpose, we use the LMD Generic Global Climate Model (GCM) whose implementation for this specific study is detailed in Sect. 2.

For further reference, Fig. 1 summarizes this attempt to quantify the possible climates of Proxima $b$ for the two most likely spin states (1:1 and 3:2 spin-orbit resonance), as a function of the total water inventory and the greenhouse gas content $\left(\mathrm{CO}_{2}\right.$ here). The total water inventory is expressed here in global equivalent layer (GEL), which is the globally averaged depth of the layer that would result from putting all the available water in the system at the surface in a liquid phase. Figure 1 serves as a guide throughout the various sections of this work.

To add a twist, Proxima b, as it is probably our closest neighbor, should be amenable to further characterization by direct imaging in the near future. With its short orbital period, multiepoch imaging could then rapidly yield a visible and NIR phase curve of the planet. It could be one of our first opportunities to characterize a temperate terrestrial planet and its climate. We thus put a particular emphasis on quantifying observable signatures for the various type of atmospheres discussed here.

After presenting the details about the physical parameterizations used to model Proxima b, Sects. 3 to 5 contain our major findings about the climate regimes achievable on Proxima $b$. They are ordered following the global water inventory available from completely dry (Sect. 3), water limited planets (Sect. 4) to water-rich worlds (Sect. 5). Finally, in Sect. 6, we highlight potential observable signatures of these various climate regimes, and discuss how direct imaging with upcoming facilities could help us to constrain the actual climate of Proxima b.

\section{Method - the LMD generic global climate model}

This model originally derives from the LMDz three-dimensional Earth GCM (Hourdin et al. 2006), which solves the primitive equations of geophysical fluid dynamics using a finite difference dynamical core on an Arakawa $\mathrm{C}$ grid. The same model has been used to study very diverse atmospheres of terrestrial planets, ranging from (1) low irradiated planets as early Mars (Forget et al. 2013; Wordsworth et al. 2013, 2015; Turbet et al. 2016), Archean Earth (Charnay et al. 2013), snowball Earth-like planets (Turbet et al., in prep.), or exoplanets like Gliese 581d (Wordsworth et al. 2011); (2) planets receiving stellar flux similar to the Earth (Bolmont et al. 2016a; this paper); and (3) highly irradiated planets such as future Earth
(Leconte et al. 2013a) or tidally locked exoplanets like Gliese 581c/HD 85512b (Leconte et al. 2013b).

Our simulations were designed to represent the characteristics of Proxima $b$, which include the stellar flux it receives $\left(956 \mathrm{~W} \mathrm{~m}^{-2} / 0.7 S_{\oplus}\right)$, its radius $\left(7160 \mathrm{~km} / 1.1 R_{\oplus}\right)$ and gravity field $\left(10.9 \mathrm{~m} \mathrm{~s}^{-2}\right)$ which are calculated assuming a mass of $1.4 M_{\oplus}$ (Anglada-Escudé et al. 2016) and the density of Earth $\left(5500 \mathrm{~kg} \mathrm{~m}^{-3}\right)$; these characteristics also include a flat topography and various rotation speeds, namely $6.3 \times 10^{-6}, 9.7 \times 10^{-6}$, and $1.3 \times 10^{-5} \mathrm{rad} \mathrm{s}^{-1}$, for $1: 1,3: 2$, and $2: 1$ orbital resonances, respectively. All the simulations were performed assuming a circular orbit. Even if the maximum possible eccentricity of Proxima b is 0.35 (Anglada-Escudé et al. 2016), for dynamical reasons (Ribas et al. 2016) the upper limit of 0.1 would be more realistic. Therefore, the mean flux approximation seems reasonable (Bolmont et al. 2016a) here. We also worked with an obliquity of $0^{\circ}$, as expected for such a planet influenced by gravitational tides (see Ribas et al. 2016, for more details).

The simulations presented in this paper were all carried out at a horizontal resolution of $64 \times 48\left(\right.$ e.g., $\left.5.6^{\circ} \times 3.8^{\circ}\right)$ in longitude $\times$ latitude. In the vertical direction, the model is composed of 26 distinct atmospheric layers that were built using hybrid $\sigma$ coordinates and 18 soil layers. These 18 layers are designed to represent either a rocky ground (thermal inertia $I_{\text {rock }}=$ $\left.1000 \mathrm{~J} \mathrm{~m}^{-2} \mathrm{~K}^{-1} \mathrm{~s}^{-\frac{1}{2}}\right)$, an icy ground $\left(I_{\text {ice }}=2000 \mathrm{~J} \mathrm{~m}^{-2} \mathrm{~K}^{-1} \mathrm{~s}^{-\frac{1}{2}}\right)$ or an ocean $\left(I_{\text {ocean }}=20000 \mathrm{~J} \mathrm{~m}^{-2} \mathrm{~K}^{-1} \mathrm{~s}^{-\frac{1}{2}}\right.$ to take into account the vertical mixing) depending on the assumed surface. Oceanic heat transport is not included in this study. Each of theses configurations is able to capture the diurnal waves for the non-synchronous orbital configurations. The planet day maximum explored duration is 22.4 Earth days for the 3:2 resonance orbital configuration. Table 1 summarizes all the parameterizations adopted in this work.

The GCM includes an up-to-date generalized radiative transfer (Rothman et al. 2009; Wordsworth et al. 2010; Richard et al. 2012) for variable gaseous atmospheric compositions made of various cocktails of $\mathrm{CO}_{2}, \mathrm{~N}_{2}$, and $\mathrm{H}_{2} \mathrm{O}$, using the correlated-k method (Fu \& Liou 1992; Eymet et al. 2016). Processes such as the radiative effect of clouds or Rayleigh scattering are taken into account. The emission spectrum of Proxima Centauri (see Fig. 2, top panel) was computed using the synthetic BT-Settl spectrum ${ }^{1}$ (Rajpurohit et al. 2013) of a M5.5 star with $T_{\text {eff }}=3000 \mathrm{~K}$, $g=10^{3} \mathrm{~m} \mathrm{~s}^{-2}$ and $[\mathrm{M} / \mathrm{H}]=0$ dex.

Around a red dwarf like Proxima b, the bolometric albedo of ice and snow is significantly reduced (Joshi \& Haberle 2012) because of the shape of its reflectance spectrum. To account for this effect, the GCM computes the bolometric albedo of ice from a simplified law of the spectral albedo of ice and snow calibrated to obtain an ice and snow bolometric albedo of 0.55 around a Sun-like star (Warren \& Wiscombe 1980; Warren 1984; Joshi \& Haberle 2012; see bottom panel of Fig. 2). Around Proxima b, our average bolometric albedo for ice and snow is 0.27 . Yet, because of the varying spectral transmission of the atmosphere (due to variable water vapor and clouds), the bolometric albedo can locally reach values as high as 0.55 .

Melting, freezing, condensation, evaporation, sublimation, and precipitation of $\mathrm{H}_{2} \mathrm{O}$ are included in the model. Similarly, we take the possible condensation and sublimation of $\mathrm{CO}_{2}$ in the atmosphere (and on the surface) into account, but not the radiative effect of $\mathrm{CO}_{2}$ ice clouds because their scattering greenhouse effect (Forget \& Pierrehumbert 1997) should not exceed 10 Kelvins in most cases (Forget et al. 2013; Kitzmann 2016).

Downloaded from https : //phoenix . ens-lyon. fr 


\section{SYNCHRONOUS ROTATION}

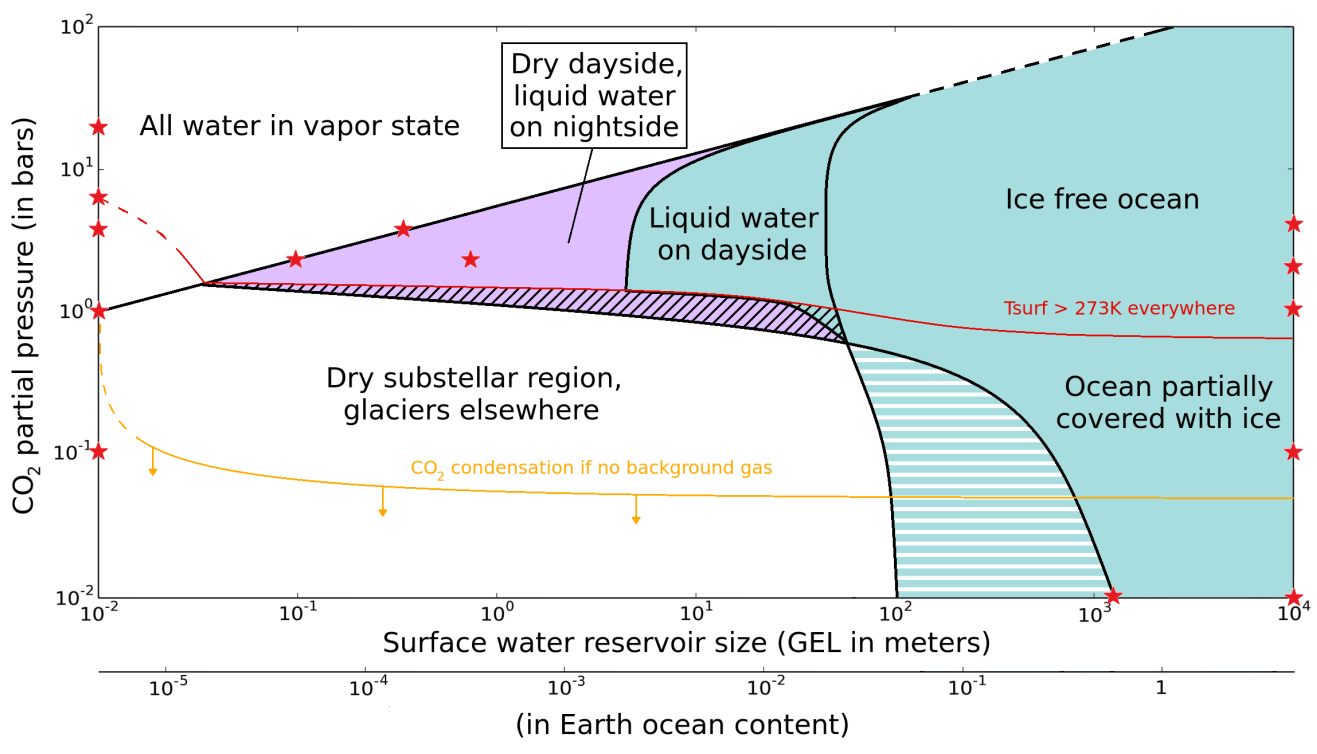

NON-SYNCHRONOUS ROTATION

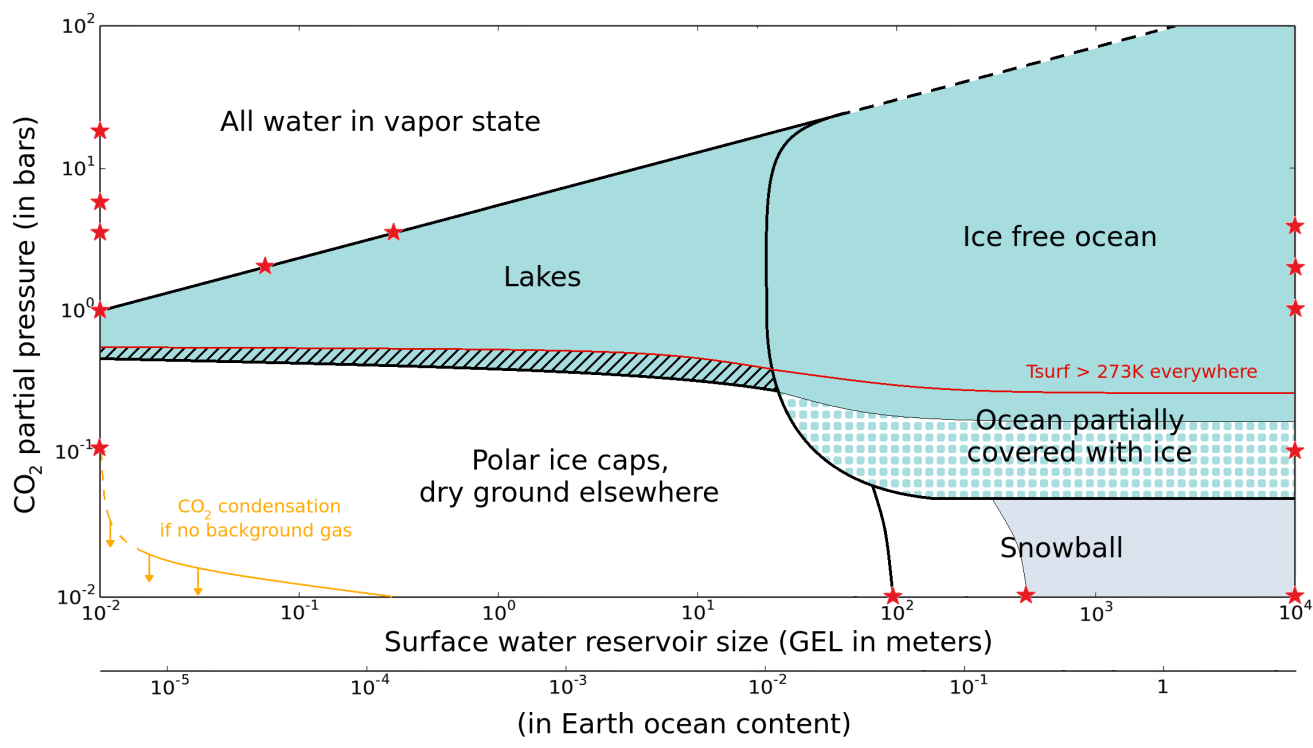

Habitable regimes :

Liquid water stable on dayside

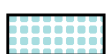

Bistability between

1) Snowball state

2) Ocean partially covered with ice

Liquid water stable only on nightside

Glacier melt locally

Bistability between

1) Water ice glaciers

2) Ocean partially covered with ice

Subsurface ocean

Global Climate Model experiments

Fig. 1. Schematic diagrams of the possible climate regimes reached as function of the $\mathrm{CO}_{2}$ atmospheric content (in bar) and the $\mathrm{H}_{2} \mathrm{O}$ content available at the surface (in global equivalent layer (GEL), in meters). The top and bottom panels describe the case of synchronous and asynchronous spin states, respectively. The red stars indicate the parts of the diagram that have been probed in this work using GCM simulations. The stars that lie on top of the left $y$-axis correspond to the case of a dry surface and atmosphere while those on the right $y$-axis consider planets completely covered by water. The presence of a background gas such as $\mathrm{N}_{2}$ could significantly modify the lower part of the diagram (pCO $\mathrm{p}_{2}<1$ bar typically). It would favor the heat redistribution, which in turn could (1) prevent the $\mathrm{CO}_{2}$ atmospheric collapse and (2) reduce the amount of ice possibly trapped in water ice glaciers. 
Table 1. Adopted stellar and planetary characteristics of the Proxima system.

\begin{tabular}{lcc}
\hline \hline Parameter & Value & Unit \\
\hline$L_{\star}$ & 0.0017 & $L_{\odot}$ \\
$T_{\text {eff }}$ & 3000 & $\mathrm{~K}$ \\
Age & 4.8 & $\mathrm{Gyr}$ \\
\hline$M_{\mathrm{p}} \sin i$ & 1.27 & $M_{\oplus}$ \\
$M_{\mathrm{p}}$ & 1.4 & $M_{\oplus}$ \\
$R_{\mathrm{p}}$ & 1.1 & $R_{\oplus}$ \\
Semi-major axis & 0.0485 & $\mathrm{AU}$ \\
$S_{\mathrm{p}}$ & 0.7 & $S_{\oplus}$ \\
Spin-orbit resonance & $1: 1 / 3: 2 / 2: 1$ & \\
$\Omega_{\mathrm{p}}$ & $0.3 \times 10^{-6} / 9.7 \times 10^{-6} / 1.3 \times 10^{-5}$ & $\mathrm{rad} \mathrm{s}^{-1}$ \\
Stellar day & $\infty / 22.4 / 11.2$ & Earth days \\
Obliquity & 0 & \\
Eccentricity & 0 & \\
\hline Surface types & Rock/Liquid water/Ice & $\mathrm{J} \mathrm{m}^{-2} \mathrm{~K}^{-1} \mathrm{~s}^{-\frac{1}{2}}$ \\
Thermal inertia & $1000 / 20000 / 2000$ & \\
Albedo & $0.2 / 0.07 /$ wavelength-dependant (Fig. 2) & \\
\hline
\end{tabular}

Notes. We also show the adopted parameters for various GCM parametrizations. The values for the stellar and planetary parameters are derived from Anglada-Escudé et al. (2016).

\section{The case of a completely dry planet}

Because Proxima b probably lost a massive amount of water during its early evolution around a pre-main sequence, active star (Ribas et al. 2016), we need to consider the possibility that the planet may now be rather dry. When water is in very limited supplies, its state is mostly determined by the temperatures of the coldest regions of the surface, the so-called cold traps (Abe et al. 2011; Leconte et al. 2013b; see next section).

To get some insight into the location and properties of these cold traps, we first consider the simple case of a completely dry planet. In this section, we focus on the greenhouse gas content needed to prevent the formation of a cold trap where ice could accumulate. Necessary conditions for the atmosphere to be stable are also discussed.

To evaluate this situation, we performed GCM simulations of rocky planets (surface albedo of 0.2 ) enveloped by pure $\mathrm{CO}_{2}$ atmospheres with pressures ranging from 0.1 bar to 20 bars. Around a red dwarf like Proxima, $\mathrm{CO}_{2}$ is a powerful greenhouse gas because it has stronger absorption lines in the near-infrared than in the visible and it does not contribute much to the stellar reflection by the Rayleigh scattering.

Figure 3 shows that the temperatures are very high across the surface even for $\mathrm{CO}_{2}$ atmospheres of moderate thickness, and despite the low insolation compared to Earth $\left(S_{\mathrm{p}}=0.7 S_{\oplus}\right)$. In fact, GCM simulations show that, whatever the atmospheric pressure or the orbital configuration, surface temperatures are always greater than $273 \mathrm{~K}$ somewhere, although it is not the most relevant factor for the stability of liquid water (and therefore habitability), as indicated by the case of current day Mars (Read \& Lewis 2004; Millour et al. 2015).

\subsection{Synchronous rotation}

For the synchronous orbit case, surface pressures of 6 bars of $\mathrm{CO}_{2}$ are required to warm the entire surface above the melting point of water. However, the surface temperature contrasts can be very high for lower atmospheric content. For example, as much as $150 \mathrm{~K}$ difference between the substellar and the coldest

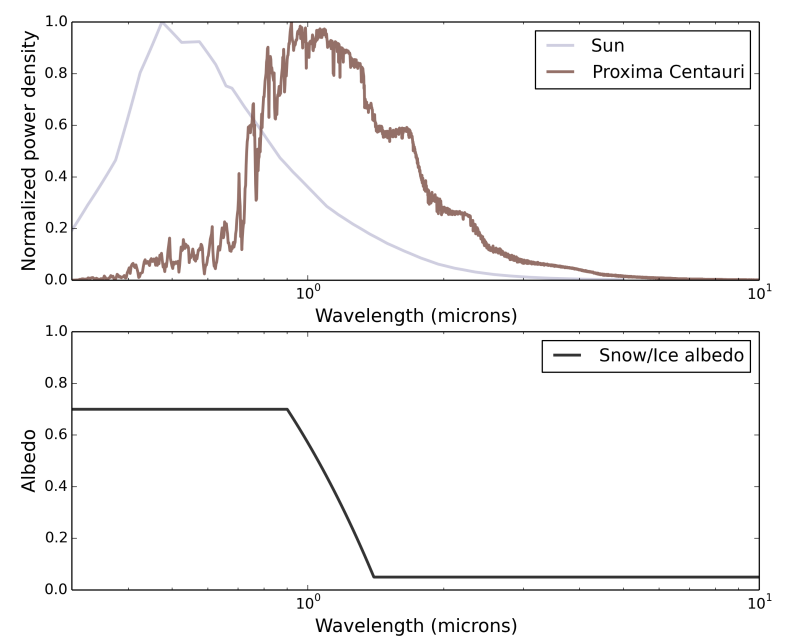

Fig. 2. Top panel: synthetic emission spectrum of a Proxima Centaurilike star (normalized by the peak value) used as input for the GCM calculations. For comparison, we put the spectrum of the Sun as usually computed in the LMD-GCM radiative transfer. Bottom panel: spectral distribution of snow and ice surface albedo as computed in the GCM. Integrated snow and ice albedo values are 0.55 and 0.27 for the Sun and Proxima Centauri, respectively.

points in the 1 bar pure $\mathrm{CO}_{2}$ simulation (see Fig. 3, left bottom corner). In the synchronous configuration, the planet has two cold points located at symmetric positions around longitude $\pm 180^{\circ}$ and latitude $\pm 60^{\circ}$. The existence of these two cold traps persists in all the tidally locked simulations (dry, wet, down to 1 bar thin or up to 20 bars thick atmospheres, etc.) explored in this work, but their position can slightly vary, as a result of planetary-scale equatorial Kelvin and Rossby wave interactions (Showman \& Polvani 2011). In particular, our simulations show that thick atmospheres tend to move these two cold gyres toward the west direction and higher latitudes (see also Fig. 6).

In addition, GCM simulations tell us that $\mathrm{CO}_{2}$-dominated atmospheres that are thinner than $\sim 1$ bar are not stable at all 
SYNCHRONOUS

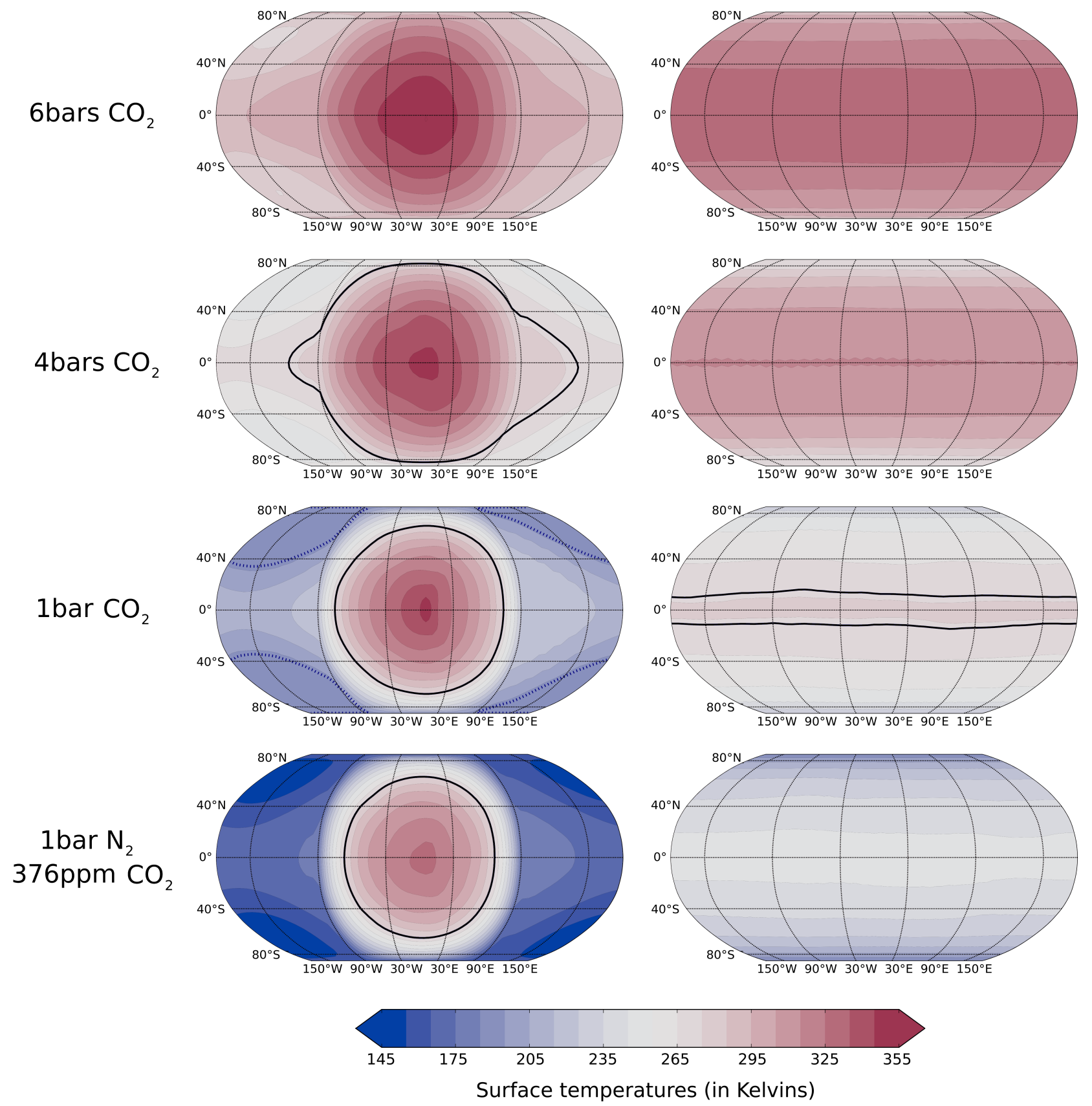

Fig. 3. Biennal mean surface temperatures of completely dry atmospheres, for 2 orbital configurations (synchronous and 3:2 orbital resonance) and 4 atmospheric compositions and pressures (6 bars of pure $\mathrm{CO}_{2}, 4$ bars of pure $\mathrm{CO}_{2}, 1$ bar of pure $\mathrm{CO}_{2}$ and 1 bar of $\mathrm{N}_{2}+376$ ppm of $\mathrm{CO}_{2}-$ Earth-like atmosphere). The solid black line contour corresponds to the $273.15 \mathrm{~K}$ isotherm; the dashed blue line contour indicates the regions where the atmospheric $\mathrm{CO}_{2}$ collapses permanently into $\mathrm{CO}_{2}$ ice deposits. The 1 bar pure $\mathrm{CO}_{2}$ (synchronous) simulation is not stable in the long term since $\mathrm{CO}_{2}$ would collapse at the two cold points.

because the surface temperature at the two cold points is lower than the temperature of condensation of $\mathrm{CO}_{2}$ (see Fig. 3, third row of left column, blue dashed line). We identified here a positive feedback: when $\mathrm{CO}_{2}$ starts to collapse because of the decrease in the total gas content, the heat redistribution becomes less efficient, which increases the temperature contrast between the substellar point and the gyres and therefore favors the $\mathrm{CO}_{2}$ condensation at the cold points. In this case, the atmosphere would inevitably collapse until reaching an extremely low $\mathrm{CO}_{2}$ atmospheric content in a regime of temperatures and pressures that are not well described by our model parametrizations.

In the process, $\mathrm{CO}_{2}$ ice could be trapped for eternity, but also form glaciers that could flow efficiently to warm regions and resupply the atmosphere in gaseous $\mathrm{CO}_{2}$ continuously (Turbet et al., in prep.). Moreover, the scattering greenhouse effect of $\mathrm{CO}_{2}$ ice clouds (Forget \& Pierrehumbert 1997; Turbet et al., in prep.) that would form preferentially in the coldest regions of the planet could drastically limit the $\mathrm{CO}_{2}$ atmospheric collapse. 
In any case, this shows that having enough atmospheric background gas (main agent of the heat redistribution + additional pressure broadening) may favor the stability of the atmosphere and therefore the habitability of Proxima $b$. For example, for an atmosphere of 1 bar of $\mathrm{N}_{2}$ - as could be more or less expected on an Earth-sized planet of $R_{\mathrm{p}} \sim 1.1 R_{\oplus}$ (Kopparapu et al. 2014) - and $376 \mathrm{ppm}$ of $\mathrm{CO}_{2}$, the dayside has mean surface temperatures above $273 \mathrm{~K}$ and the atmosphere does not collapse (see Fig. 3, left bottom corner).

\subsection{Asynchronous rotation}

For non-synchronous cases, the substellar temperature "eye" pattern disappears and the atmospheric pressure at which surface temperatures are all strictly above $273 \mathrm{~K}$ is slightly lower because the stellar radiation is now distributed equally among the longitudes. For the 3:2 resonance case, Fig. 3 shows that this condition is reached for atmospheric pressures greater or equal to 4 bars. 3:2 and 2:1 spin-orbit resonance configurations do not exhibit significant differences in term of surface temperature maps. The shorter stellar day in 2:1 (11.2 Earth days compared to 22.4), which weakens day and night contrasts is compensated by the higher rotation rate, which weakens equator-to-pole heat redistribution (Wordsworth et al. 2011; Kaspi \& Showman 2015).

Another crucial consequence of the efficiency of the heat redistribution relates to the $\mathrm{CO}_{2}$ collapse, which occurs now at $\mathrm{CO}_{2}$ atmospheric pressure as low as 0.1 bar in the 3:2 orbital resonance GCM simulations (this is a factor 10 lower than for the tidally locked configuration). Therefore, in such a configuration, asynchronous rotation would favor the stability of the atmosphere and thus the habitability of Proxima $b$.

\section{Limited water reservoir}

Another possibility is that Proxima b may have a limited, but non-zero, water inventory. In this case, the question is to know where this water is stored. To answer that question, Sect. 4.1 first makes an attempt at quantifying how much water vapor can be stored in the atmosphere without ever condensing at the surface. Then, in Sect. 4.2, we discuss what happens to the water reservoir when it condenses at the surface. In particular, we try to estimate how much water can be stored before it forms planetary scale oceans (this is discussed in Sect. 5).

\subsection{Maximum amount of water stored in the atmosphere}

The amount of water that can be maintained in a planetary atmosphere before it starts to condense at the surface depends primarily on the atmospheric temperatures. For example, Venus has a water GEL of 2 cm (Bougher et al. 1997; Bézard et al. 2009) in its atmosphere that may form liquid water droplets in the atmosphere but never condenses at the surface. But it could store much more.

To quantify this possibility in the case of Proxima $b$, we perform a simulation with a 4 bars $\mathrm{CO}_{2}$ dominated atmosphere, for a synchronous rotation, in aquaplanet mode (static ocean model, $I=20000 \mathrm{~J} \mathrm{~m}^{-2} \mathrm{~K}^{-1} \mathrm{~s}^{-\frac{1}{2}}$, surface albedo of 0.07 ), and until equilibrium is reached (after $\sim 1000$ orbits). Hereafter, water vapor is always included in the atmospheric calculations, expect when otherwise specified. When referring to a specific atmospheric composition, only the amount of background gases is specified.
Suddenly, we change the properties of the planet: first, we remove all the water available at the surface and, second, we use a rocky surface $\left(I=1000 \mathrm{~J} \mathrm{~m}^{-2} \mathrm{~K}^{-1} \mathrm{~s}^{-\frac{1}{2}}\right.$, surface albedo of 0.2), in place of the removed water at the surface. Then, at every orbit, we remove all the extra water that condenses from the planet. We repeat the process until precipitations completely stop for 50 consecutive orbits. Up to $45 \mathrm{~cm}$ GEL of water could be trapped in the atmosphere before it starts to condense (not shown). In this case, surface temperatures reach 370-450 K, which is a $45 \mathrm{~K}$ increase on average compared to the aquaplanet simulation.

Repeating this procedure for atmospheric pressures of 1 bar, 2 bars, and 4 bars, we obtained maximum amounts of water vapor (before it condenses permanently at the surface) of $1 \mathrm{~cm}$, $10 \mathrm{~cm}$, and $45 \mathrm{~cm}$ GEL. The corresponding simulations were reported in Fig. 1 and define the black curve separating the region "All water in vapor state" from the rest of the diagram. The same GCM simulations, when taking the greenhouse effect of water vapor into account, show that surface pressures as low as 1.5 bars (and even lower for the 3:2 orbital resonance) are now sufficient to get surface temperatures above $273 \mathrm{~K}$ everywhere on the planet.

Thick, Venus-like atmospheres $\left(\mathrm{pCO}_{2}\right.$ typically $>10$ bars) could potentially store very large amounts of water, although we did not perform the necessary simulations; these simulations would require a dedicated radiative transfer model. Interestingly, for atmospheres with a huge greenhouse effect, there is no reason for the partial pressure of water not to exceed that of the critical point. In this case, the temperature would also exceed the critical point so that discussing the transition to a liquid phase at the surface would not be very meaningful. The habitability of such environment seems largely unexplored

\subsection{Maximum amount of water stored on the surface}

\subsubsection{Cold climates: limits on glaciers}

As soon as water is available in sufficient amounts, which depends on the atmospheric gas content (as detailed above), it can start to condense permanently at the surface. For a synchronous rotation, $\mathrm{CO}_{2}$-dominated atmospheres with pressure typically lower than 1.5 bars exhibit surface temperatures that are lower than $273 \mathrm{~K}$ at their coldest points. Therefore, all the extra water available at the surface gets trapped at the two cold gyres forming inevitably stable water ice deposits. The range of water inventory for which such unhabitable climate regimes subsist depends on the atmospheric gas content and composition, but also possibly on the internal heat flux of Proxima Centauri b.

As the water inventory increases, the ice deposits thicken and start to form water ice glaciers that can at some point flow from the coldest regions of the planet toward warmer locations. There are in fact two distinct processes that can limit the growth of water ice glaciers (Leconte et al. 2013b; Menou 2013):

1. The gravity pushes the glaciers to flow in the warm regions where ice can be either sublimated or melted. This limit depends mostly on the gravity of the planet and the mechanical properties of water ice (e.g., viscosity).

2. The internal heat flux of the planet causes the basal melting of the water ice glaciers. In such conditions, glaciers would slip and flow to warmer regions where, once again, ice could melt and sublimate. This limit depends primarily on the geothermal heat flux of the planet and the thermodynamical properties of water ice (e.g., thermal conductivity). 
SYNCHRONOUS

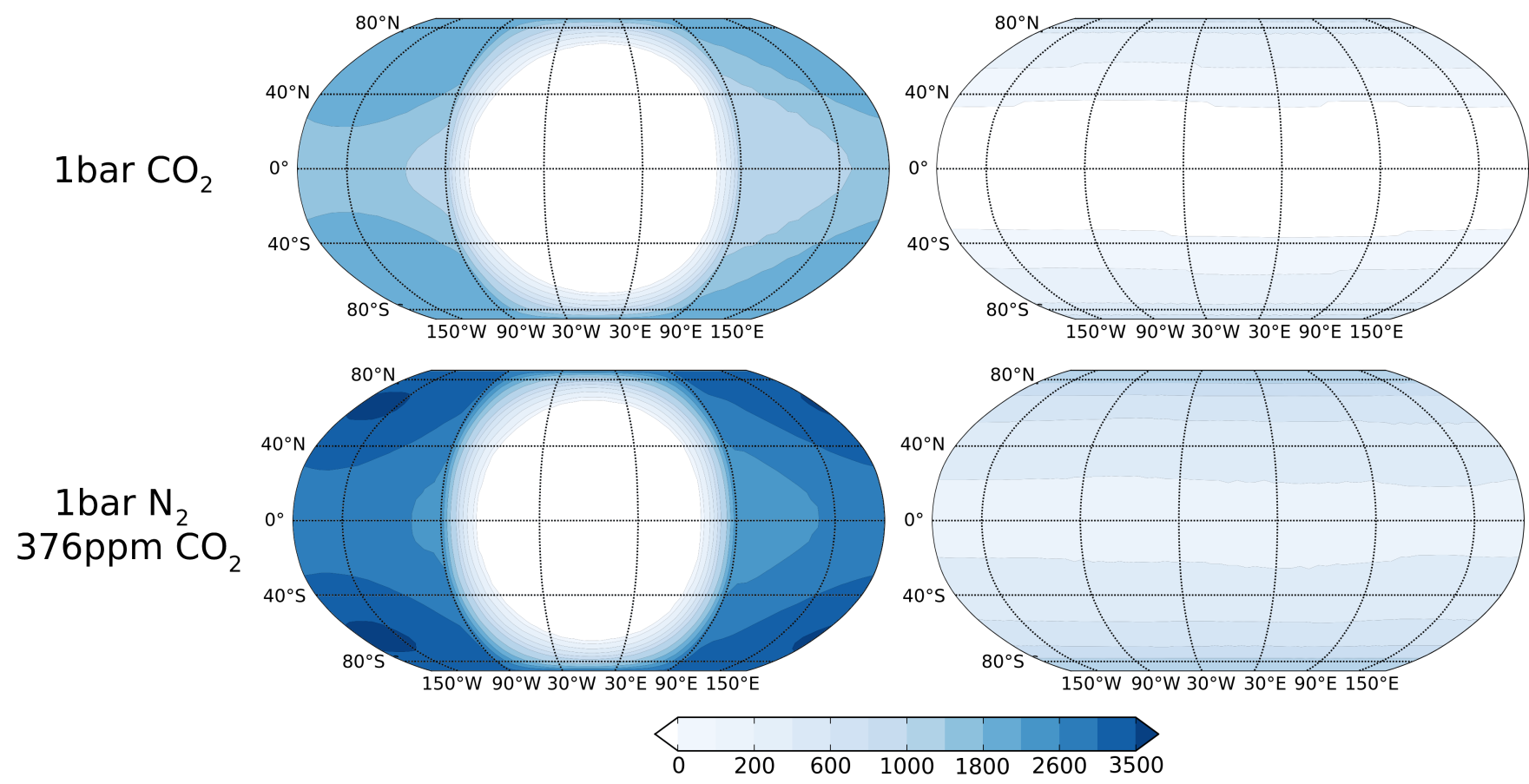

Maximum ice thickness (in meters)

Fig. 4. Maximum ice thickness calculated from 4 completely dry GCM simulations made of 2 different atmospheric compositions ( 1 bar pure $\mathrm{CO}_{2}$ atmosphere, 1 bar $\mathrm{N}_{2}$-dominated atmosphere with $376 \mathrm{ppm}$ of $\mathrm{CO}_{2}$ ) and 2 orbital configurations (synchronous and 3:2 resonance). The maximum ice thickness is calculated from the basal melting condition derived from the mean surface temperature from Fig. 3. The internal heat flux is assumed to be $110 \mathrm{~mW} \mathrm{~m}^{-2}$ for the synchronous orbit cases and $180 \mathrm{~mW} \mathrm{~m}^{-2}$ for the asynchronous cases. The global equivalent thickness of ice is $940 \mathrm{~m}\left(1 \mathrm{bar}\right.$ of $\left.\mathrm{CO}_{2}\right)$ and $1650 \mathrm{~m}$ (Earth atmosphere) for the synchronous rotation, and $115 \mathrm{~m}$ and $490 \mathrm{~m}$ for the 3:2 resonance, respectively. The amount of ice calculated from the 1 bar $\mathrm{CO}_{2}$ simulation is probably a lower estimate since $\mathrm{CO}_{2}$ would collapse, making the cold points even colder.

Menou (2013) has shown that for tidally locked terrestrial planets with Earth-like characteristics, the basal melting should be the condition that limits the thickness of the water ice glaciers. He finds maximum global equivalent ice thicknesses typically ranging from $320 \mathrm{~m}$ (for a 1 bar $\mathrm{N}_{2}, 3.6 \% \mathrm{CO}_{2}$ atmosphere) and $770 \mathrm{~m}$ (0.3 bar $\mathrm{N}_{2}, 360 \mathrm{ppm} \mathrm{CO}_{2}$ atmosphere).

In the same vein, we compute the maximum ice thickness before basal melting for four of our dry GCM simulations from Fig. 3. We use two distinct atmospheric compositions: 1 bar is pure $\mathrm{CO}_{2}$ atmosphere; below that, the $\mathrm{CO}_{2}$ can collapse permanently for synchronous rotation; 1 bar of $\mathrm{N}_{2}+376 \mathrm{ppm}$ of $\mathrm{CO}_{2}$ ), and two orbital configurations (synchronous and 3:2 resonance). This thickness, $h_{\text {ice }}^{\max }$, is given by Abbot \& Switzer (2011)

$h_{\text {ice }}^{\max }=\frac{A}{F_{\text {geo }}} \ln \left(\frac{T_{\text {melt }}}{T_{\text {surf }}}\right)$,

where $F_{\text {geo }}$ is the internal heat flux and $T_{\text {melt }}$ is the melting temperature of ice at the base of the glacier. As the latter is a function of the pressure below the ice, it implicitly depends on $h_{\text {ice }}^{\max }$ so that the above equation must be solved numerically, once the local surface temperature is known (Leconte et al. 2013b; see Appendix A for details). This temperature is taken from the GCM outputs (see the third and fourth rows of Fig. 3).

Assuming that it scales roughly with $M_{\mathrm{p}}^{1 / 2}$ (Abbot \& Switzer 2011), the geothermal flux can be extrapolated based on the Earth value ( $90 \mathrm{~mW} \mathrm{~m}^{-2}$; Davies \& Davies 2010), yielding
$F_{\text {geo }}=110 \mathrm{~mW} \mathrm{~m}^{-2}$. Of course, this estimate holds only because the Earth and Proxima are similar in age (Bazot et al. 2016). However, tidal heating could also take place. Ribas et al. (2016) showed that an initial eccentricity would not be damped significantly over the lifetime of the system. They also argue that, assuming the planet is alone in the system, it would be difficult to excite the orbital eccentricity above 0.1 . This would correspond to an extra tidal heat flux of $\sim 70 \mathrm{~mW} \mathrm{~m}^{-2}$ for a tidal dissipation ten times lower than Earth. Therefore, for nonsynchronous orbits, we arbitrarily set the geothermal heat flux $F_{\text {geo }}$ to be equal to $110+70=180 \mathrm{~mW} \mathrm{~m}^{-2}$. An upper limit on the tidal heating can also be derived from observations of Proxima $b$ that put an upper limit of 0.35 on the eccentricity of the planet (Anglada-Escudé et al. 2016). This configuration produces a tidal dissipation heat flux of $\sim 2.5 \mathrm{~W} \mathrm{~m}^{-2}$, which is similar to Io (Spencer et al. 2000). Yet, in this case, most of the heat would probably be extracted through convection processes (e.g., volcanism) instead of conduction, as on Io. Thus only a (unknown) fraction of this flux should be used in Eq. (1). We thus decided to use a geothermal flux of $180 \mathrm{~mW} \mathrm{~m}^{-2}$ for our baseline scenario, but we note that an order of magnitude change could be possible.

Figure 4 shows the water ice maximum thickness maps derived for our four simulations. After spatial averaging, this yields maximum equivalent global thicknesses of ice of $940 \mathrm{~m}$ and $115 \mathrm{~m}$ for the 1 bar pure $\mathrm{CO}_{2}$ atmosphere (respectively for sync. 
and async. rotations) and of $1650 \mathrm{~m}$ and $490 \mathrm{~m}$ for an Earth-like atmosphere.

On the one hand, very large amounts of ice (up to $61 \%$ of Earth ocean content in the Earth-like atmosphere config.) can be trapped in the tidally locked case due to the high contrasts of temperature throughout the surface. This is not an upper limit. Thinner atmospheres (due to $\mathrm{CO}_{2}$ collapse for example, see Sect. 3) could entail much more extreme surface temperature contrasts. Such a Pluto-like planet could potentially trap tremendous amounts of water in the form of ice.

On the other hand, much more limited quantities of ice can be trapped in asynchronous simulations due to both a better heat redistribution and a higher geothermal heat flux. For an eccentricity of 0.35 , the amount of trapped ice would be probably much less than $115 \mathrm{~m}$ and $490 \mathrm{~m}$ because of the increased internal heat flux.

We mention that these GCM simulations were performed with a dry atmosphere; the lack of water vapor, a powerful greenhouse gas, leads to an overestimate of the amount of ice possibly trapped.

Eventually, once the water ice glaciers start to spill, they can possibly melt at their edge, either on dayside or nightside. It has been shown by Leconte et al. (2013b) that such configuration could be long lived. We roughly put in Fig. 1 (hatched region) the range of $\mathrm{CO}_{2}$ and water inventory for which such scenario would happen. This is an exotic form of habitability.

\subsubsection{Warm climates: appearance of lakes}

As discussed in Sect. 3, if the greenhouse effect of the atmosphere is sufficient, the coldest temperatures at the surface are above the freezing point of water. Although we might intuitively think that this situation is very different from above, from the point of view of the atmosphere, it is not. The atmosphere always tends to transport water from the hottest to the coldest regions. The fact that the cold-trap temperature is above the freezing point is irrelevant as long as there is not enough water at the surface to redistribute water more rapidly than it is brought in.

Liquid water thus first accumulates around the coldest regions of the planet. Interestingly, on a synchronous planet, these are located on the night side where no photons are available for photosynthesis. If the spin is non-synchronous, the volatiles would likely first concentrate toward the poles. This seems to be the case on Titan where methane lakes are mostly seen at high latitudes (Stofan et al. 2007).

The range of water inventory for which this configuration (liquid water on nightside) may subsist not only depends on the water inventory but also on the topography. In the case of a tidally locked planet, the topography features may not be randomly distributed because tidal locking could tend to favor the alignment of large-scale gravitational anomaly (correlated with topography anomaly) with the star-planet axis. For instance there is a clear difference between the near side and far side of the Moon (Zuber et al. 1994), and the deep Sputnik Planum basin on Pluto is located near the anti-Charon point (Moore et al. 2016). Thus it is conceivable that Proxima b may have its largest topographic basin either near the substellar point or the anti-stellar point.

Interestingly, as the water inventory grows, the response of the climate (amount of water vapor, atmospheric temperatures, ...) might be significantly different depending on the topography. Figure 5 illustrates qualitatively the fact that, depending on the topographic setup (basin at the substellar point, basin at the antistellar point, or no basin at all, i.e., a quasi-flat configuration),

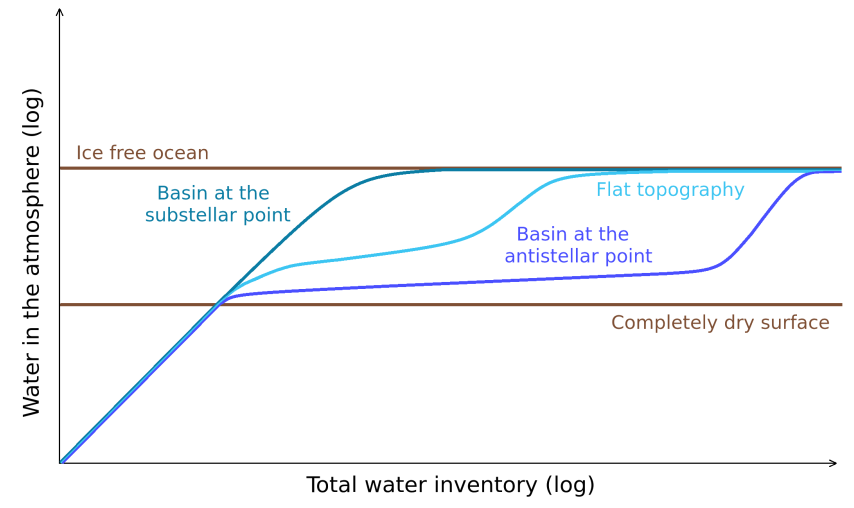

Fig. 5. Qualitative evolution of the amount of water vapor in the atmosphere as a function of the total water inventory, assuming a synchronous rotation and a liquid water runoff activated, for three different scenarios: a quasi-flat topography, a basin at the substellar point, and a basin at the anti-stellar point. For high $\mathrm{pCO}_{2}$, the range of water inventory for which the three scenarios diverge would flatten on a logarithmic scale because potentially high amount of water could be vaporized.

the distribution of water between the surface and the atmosphere might significantly differ. As significant amounts of water start to condense at the surface, liquid water would likely spill toward the main topographic depression of the surface. If this depression is for example located at the substellar point, where evaporation rates are the highest, the proportion of water in the atmosphere given a fixed total water inventory would be maximum and definitely much higher than in the extremely opposite case (a basin at the anti-stellar point).

Therefore, it is important to mention that assessing the proportion of water vapor in the atmosphere of Proxima $b$ might not be sufficient to get information on the stability and location of surface liquid water, and vice versa, knowing the exact water inventory available on Proxima b would not be totally relevant to deduce its possible climatic regime.

\section{Large water reservoir}

Despite the large amount of hydrogen that could have escaped within the lifetime of Proxima $b$, the quantity of water now available on the planet also depends significantly on its initial water inventory. As argued by Ribas et al. (2016), if Proxima Centauri $b$ formed beyond the ice line in a similar fashion to solar system icy satellites, it could still possess enough water to be an aquaplanet, the term aquaplanet refering here to a planet where water is abundant enough to flow efficiently on a planetary scale. In practice, in our simulations, this means that the surface acts as an infinite source of water.

In this section, we thus make an attempt at quantifying how much is enough. Then, we discuss the various climate regimes available to an aquaplanet and point out the main differences with the dry case.

\subsection{Transition from small to large water inventory}

The exact water inventory for which Proxima $b$ would transition from a land planet (previous section) to an aquaplanet (this section) is difficult to define. In particular, the nature of the transition depends of the amount of greenhouse gas in the atmosphere.

For a low atmospheric greenhouse effect (meaning that ice is stable somewhere on the planet), there is a bistability between 
two possible climate regimes around the transition. To understand this bistability, we introduce a thought experiment in which we consider a planet with two very different initial conditions:

- Case 1: warm- and/or water-rich-start. We assume that the planet starts with a global ocean, possibly covered by sea ice wherever cold enough. This experiment has been carried out by Yang et al. (2014) with a $325 \mathrm{~m}$ deep ocean. They showed that in such a configuration, winds carry sea ice toward hot regions and the ocean carries heat toward cold regions, so that an equilibrium can be found with less than $10 \mathrm{~m}$ of sea ice in the coldest regions. They however acknowledge that the presence of continents could significantly alter this value. Yang et al. (2014) only looked at a synchronous planet, but the argument remains qualitatively valid for a non-synchronous planet, even though numerical values will surely change significantly.

Therefore, water content as small as $\sim 10^{2} \mathrm{~m} \mathrm{GEL}$ (and maybe even lower) is sufficient to maintain aquaplanet conditions. If water were to be removed or the temperature decreased, at some point, the oceanic transport would shut down and the planet would transition to a dry, cold regime with glaciers.

- Case 2: cold- and/or water-poor-start. As discussed in the previous section, if the planet started cold or dry enough, all the water would be trapped in ice caps and glaciers. Now, we demonstrated earlier that in this state, more than $\sim 10^{3} \mathrm{~m}$ GEL of ice could be stored this way. If the water inventory and/or the temperature were to be increased, glaciers would progressively spill toward hotter regions. This state could resist until the oceanic transport becomes more efficient than the atmospheric transport. After that, an ocean would accumulate and, in turn, warm the cold regions, speeding up the transition to the aquaplanet regime.

Therefore, between roughly $\sim 10^{2}$ and $10^{3} \mathrm{~m}$ GEL, two distinct climate regimes coexist, depending on the history of the planet. As both the water inventory and the amount of greenhouse gases play a role, it is even possible for the planet to undergo hysteresis cycles between the two states. But the mechanism is rather different from that involved in snowball climates (which will be discussed later on). Indeed, here, the albedo feedback does not play a major role. What controls the transition between a water-rich and warm world toward a dry and cold one is now the oceanic transport. We note that on a non-synchronous planet, the upper limit of $10^{3} \mathrm{~m}$ GEL would decrease as the cold traps are less efficient and at the same time, a smaller global ocean might be needed to efficiently warm the poles.

For warmer climates (in the sense that ice cannot form at the surface), the transition would happen whenever water were abundant enough to flow. Topography would thus be the key parameter (see Sect. 4.2.2).

A third situation can occur when the amount of greenhouse gases in the atmosphere is such that the partial pressure of water vapor at the surface can exceed that of the critical point. Then we expect no transition at all as there is no phase transition between liquid and gaseous phase above the critical point.

Finally, we note that in any case another transition would occur at much higher water contents $\left(\sim 10^{5-6} \mathrm{~m}\right.$ GEL$)$ when high pressure ices form.

\subsection{Necessary conditions to have surface liquid water}

Assuming that Proxima $\mathrm{b}$ is in the aquaplanet regime, one may wonder what the minimal requirement is to maintain liquid water stable at the surface. Compared to the limited reservoir case, the requirements are much less stringent. There are two main reasons for this:

- There is too much water, by definition, for complete coldtrapping to occur. As a result, the lowest temperature at the surface is rather irrelevant. Instead, only the highest temperature matters. And it is much easier to have a planet with one region above freezing than a planet with no region below freezing.

- Because water is readily available for evaporation, the atmosphere, on average, is much closer to saturation compared to the dry case. As water vapor is a good greenhouse gas, this usually entails that for a given background atmosphere, the average surface temperature of the aquaplanet is higher than its dry counterpart. This can sometimes be mitigated by the ice albedo effect, but this effect is extremely weak around red stars such as Proxima (Joshi \& Haberle 2012), especially for synchronous spin states.

With that in mind, we performed a suite of aquaplanet simulations with various atmospheric compositions to assert the likelihood of surface liquid water. The results are shown in Fig. 6.

\subsubsection{Synchronous rotation: Ubiquity of liquid water.}

As can be seen on the left column of Fig. 6, when the planet is synchronously rotating, temperatures are always high enough at the substellar point to have liquid water, whatever the atmospheric content (pure $\mathrm{CO}_{2}$, Earth-like mixture, thin, 0.01 bar atmosphere). This would hold even without a background atmosphere; in this case, the atmosphere would be composed of water vapor. Indeed, because the substellar point permanently receives $956 \mathrm{~W} \mathrm{~m}^{-2}$, it would take a surface bond albedo of 0.67 to cool this region below the freezing point. We recover the "eyeball Earth" regime (Pierrehumbert 2011) or "Lobster Earth" regime (Hu \& Yang 2014, when deep ocean circulation is taken into account).

Starting from there, adding greenhouse gases to the atmosphere only (1) increases the mean surface temperature, (2) increases the size of the liquid water patch, and (3) reduces the temperature contrast. Eventually, above some threshold ( $\sim 1$ bar in our simulations), greenhouse is intense enough to deglaciate the whole planet.

\subsubsection{Asynchronous rotation}

In a non-synchronous spin-state, on the contrary, surface liquid water is not always possible. In fact, we recover a situation very similar to that on Earth: below some threshold amount of greenhouse gases, the planet falls into a frozen, snowball state.

The reason for this difference with the synchronous state is that any point at the equator now receives on average only $1 / \pi$ of what the substellar point received at any time in the synchronous configuration. The zero-albedo equilibrium temperature corresponding to this mean flux is $\sim 270 \mathrm{~K}$. Some greenhouse effect is thus necessary to melt the equator, especially when the albedo and circulation effects are added.

Consequently, four different climate regimes can be reached on such an asynchronous planet, depending on the greenhouse gas content. These are depicted on the right column of Fig. 6. From top to bottom, we have

1. For high $\mathrm{CO}_{2}$ pressures (roughly above a few tenths of bars) the planet is covered by an ice-free ocean. We did not 


\section{SYNCHRONOUS}

1 bar $\mathrm{CO}_{2}$

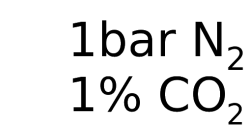

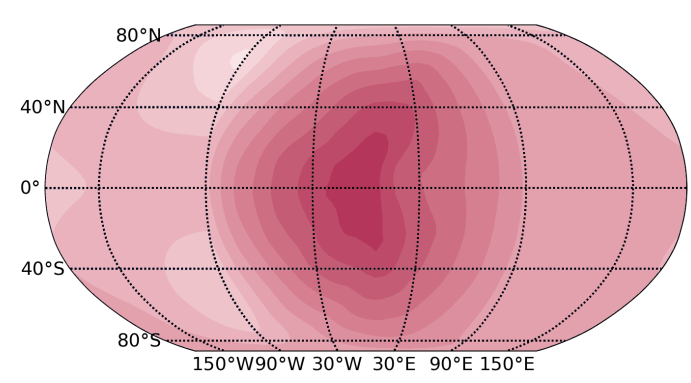

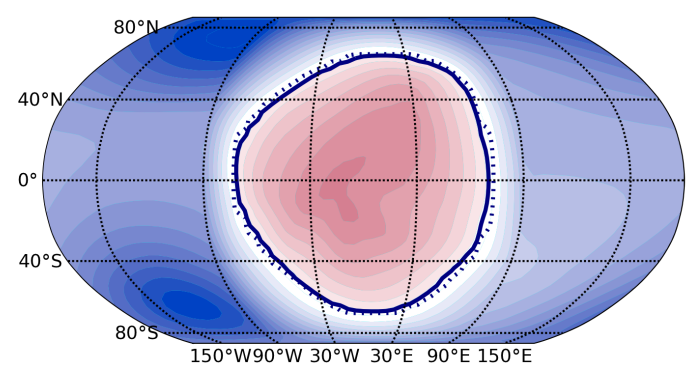

$50^{\circ} \mathrm{W} 90^{\circ} \mathrm{W} 30^{\circ} \mathrm{W} 30^{\circ} \mathrm{E} 90^{\circ} \mathrm{E} 150^{\circ} \mathrm{E}$

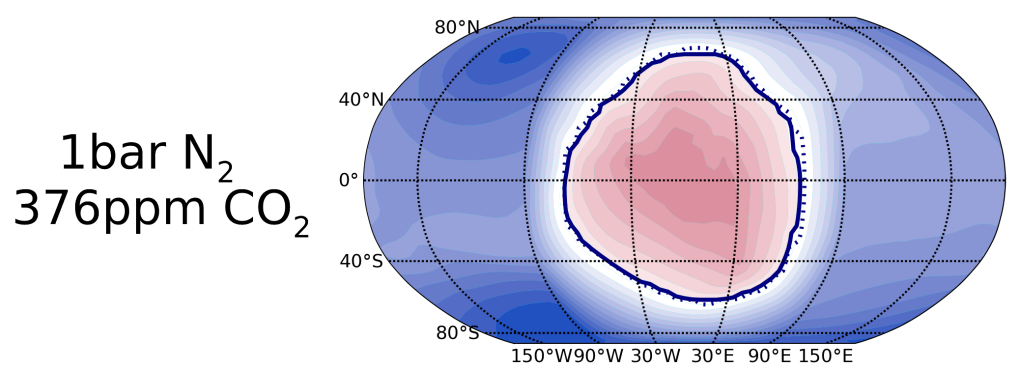

0.01 bar $\mathrm{N}_{2}$
$376 \mathrm{ppm} \mathrm{CO}_{2}$

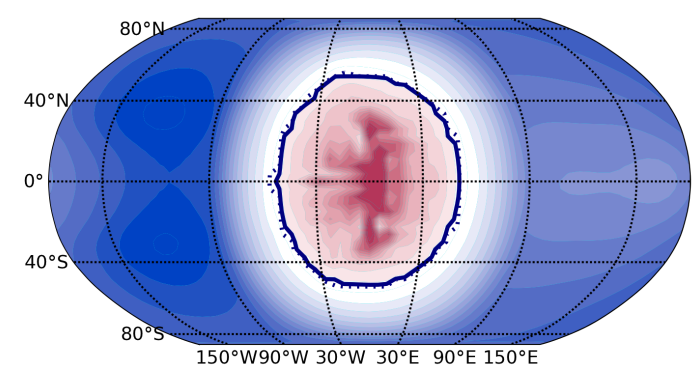

\section{3:2 RESONANCE}
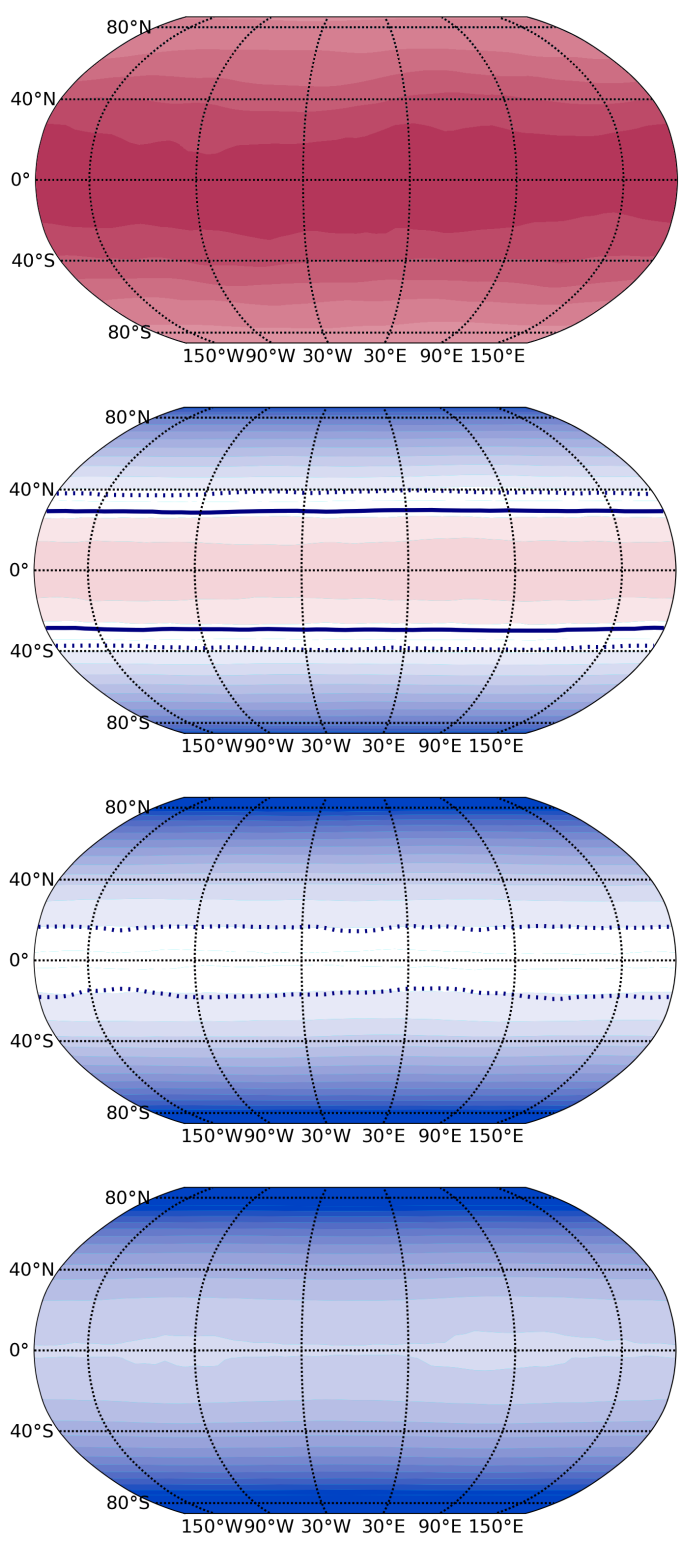

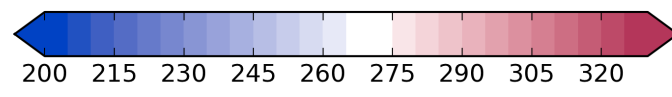

\section{Surface temperatures (in Kelvins)}

Fig. 6. Biennal mean surface temperatures of atmospheres made of a variety of 4 cocktails of $\mathrm{N}_{2}$ and $\mathrm{CO}_{2}$ for 2 orbital configurations (synchronous and resonance 3:2), assuming planets initially completely covered by liquid water. Solid lines and dashed lines contours correspond to the location of permanent and seasonal surface liquid water, respectively.

perform our simulations on a fine enough grid to be more precise, but the limit should be lower than 1 bar (see Fig. 6).

2. At lower levels of $\mathrm{CO}_{2}$ (down to $\sim 0.01$ bar with 1 bar $\mathrm{N}_{2}$ ) the planet can keep a permanently unfrozen equatorial belt.

3. For lower greenhouse gas contents, diurnal patches of liquid water lagging behind the substellar point subsist.

4. For thin enough atmospheres, a completely frozen snowball state ensues.

The 0.01 bar atmosphere (in Fig. 6, right column) is colder than 1 bar because, first, the pressure broadening of $\mathrm{CO}_{2}$ absorption lines by $\mathrm{N}_{2}$ is drastically reduced; and, second, the absolute amount of $\mathrm{CO}_{2}$ in the atmosphere is 100 times lower. Both effects are responsible for the appearance of an equatorial band of relatively warmer surface temperatures for the 3:2 resonance, 1 bar of $\mathrm{N}_{2}\left(+376\right.$ ppm of $\left.\mathrm{CO}_{2}\right)$ case.

The well known snowball hysteresis could potentially exist between our states 2, 3, and 4, although the weak ice-albedo feedback around $\mathrm{M}$ dwarfs certainly makes it less likely than on Earth (Shields et al. 2014). A confirmation of this would necessitate numerous additional simulations. 


\subsection{Subsurface oceans?}

Whether or not surface liquid water is possible, we now try to assess the possibility of the presence of a subsurface ocean. Indeed, if Proxima $b$ has been able to keep a large enough water inventory, the steady release of geothermal heat entails a rise in temperature with depth through the ice cap.

To assess a lower limit, we consider our coldest, and only fully glaciated case $\left(0.01\right.$ bar of $\mathrm{N} 2,376 \mathrm{ppm}$ of $\mathrm{CO}_{2}$ and 3:2 resonance; see Fig. 6, right bottom corner). Following a similar approach to Sect. 4.2.1, we estimate that a subsurface ocean could exist for water GELs greater than $600 \mathrm{~m}$ (assuming a geothermal heat flux of $180 \mathrm{~mW} \mathrm{~m}^{-2}$ ). Any effect warming the surface would tend to lower this threshold.

Above such water inventories, Proxima $b$ could thus be at least considered a class III or IV (if there is enough water to form high pressure ices) habitable planet (Lammer et al. 2009; Forget 2013).

\subsection{Thin atmospheres: implication for water loss}

When considering water loss around planets in the habitable zone of small stars, it is tempting to disregard water losses occurring after the end of the initial runaway greenhouse phase (which can be important, e.g., Ribas et al. 2016). The reason for this is that the tropopause usually acts as an efficient cold trap. The amount of water vapor available for escape in the upper atmosphere is thus limited by diffusion (Kasting et al. 1993; Wordsworth \& Pierrehumbert 2013).

However, this conclusion is often based on calculation including a relatively massive, Earth-like background atmosphere. Because escape may have been very important for Proxima $b$ in the past, early atmospheric escape may have removed an important fraction of the background atmosphere (Ribas et al. 2016). It is thus primordial to infer whether a less massive background atmosphere is still able to shield water vapor from escape once the planet has cooled down.

To that purpose, we performed simulations with an Earthlike atmospheric composition, but a lower background surface pressure (namely 0.1 and 0.01 bar; bottom panels of Fig. 6). The substellar temperature and vapor mixing ratio profiles are shown in Fig. 7, along with the reference 1 bar case.

The water vapor mixing ratio increases drastically in the atmosphere when the background pressure decreases, even at a given pressure level. This results from the fact that: (1) when the surface pressure decreases, the surface temperature cannot change drastically to remain in radiative equilibrium; (2) throughout the troposphere, the temperature follows a (moist) adiabat and is thus determined by the ratio of the local to the surface pressure. At a given pressure level, temperatures in the troposphere thus increase when the surface pressure decreases; (3) because of the Clausius-Clapeyron law, this increases the mixing ratio of water vapor throughout the troposphere; and (4) finally, the strong absorption bands in the near-infrared, i.e., the peak of the stellar spectrum, provide a positive feedback that tend to humidify the tropopause even more.

Moreover, the water vapor mixing ratio increases globally because it is advected by the large-scale circulation. As a consequence, hydrogen escape is not limited by the diffusion of water vapor anymore, even after the runaway phase. Low atmospheric background gas contents may thus lead to increased rates of hydrogen (and thus water) loss to space.
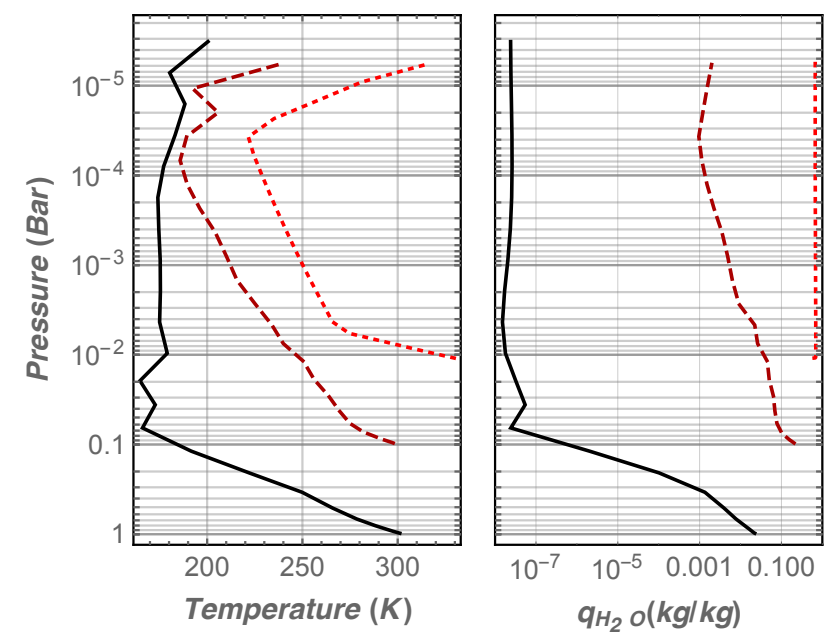

Fig. 7. Annual mean atmospheric temperature (left) and water vapor (right) vertical profiles of atmospheres with different total surface pressure (solid black: 1 bar; dashed, dark red: 0.1 bar; dotted red: 0.01 bar). The composition of all three atmospheres is $\mathrm{N}_{2}$ with $376 \mathrm{ppm}$ of $\mathrm{CO}_{2}$ and a variable amount of water vapor. The profiles are shown at the substellar point, but horizontal variations are fairly small above the 0.1 mbar level due to an efficient transport. Decreasing the surface pressure increases the water vapor ratio in the upper atmosphere drastically.

\section{Observability}

At the time of this study, the existence of planet transits or flares have not been established, which complicates the search. There is only $1.3 \%$ of chance that the inclination of the orbit of Proxima $b$ produces transits, so we do not consider characterization by transit spectroscopy in this work.

\subsection{Prospects for direct imaging}

Proxima $b$ may be the habitable-zone terrestrial exoplanet offering the best combination of angular separation and contrast for imaging. The angular separation between the planet and its star varies from 0 (for a $90^{\circ}$ inclination) to 38 mas $(0.05 \mathrm{AU}$ at $1.29 \mathrm{pc})$. The planet/star contrast of a $1.1 R_{\oplus}$ purely Lambertian sphere (surface albedo $=1$ ) at $0.05 \mathrm{AU}$ from Proxima is $2 \times 10^{-7}$ when seen with a $90^{\circ}$ phase angle and approaches $6 \times 10^{-7}$ as the phase angle approaches $0^{\circ}$. Current instrumentation using adaptive optics and coronography on $10 \mathrm{~m}$ class telescopes (such as SPHERE/VLT, GPI/Gemini) aims to achieve a contrast of $10^{-6}-10^{-7}$, but with an inner working angle of a few $\lambda / D$ that is not smaller than 100-200 mas depending on the band (Lawson et al. 2012). Lovis et al. (2016) suggest that the detection can actually be achieved with VLT by coupling SPHERE with the future high-resolution spectrometer ESPRESSO (first light expected in 2017). The idea is to first use SPHERE to reduce the stellar light by a factor $10^{3}-10^{4}$ at 37 mas from Proxima $(\sim 2 \lambda / D$ at $700 \mathrm{~nm})$ and then to search with ESPRESSO for the Doppler shifted planet signature on the $\sim 37$ mas-radius annulus around the star when RV ephemeris predict maximal angular separation. The planet and stellar signals could indeed be separated thanks to cross-correlations with molecular highresolution fingerprints, which could be specific to the planet, such $\mathrm{O}_{2}$, or reflected and Doppler shifted in both cases by the planetary orbital motion. Such a planet-star disentangling was already achieved for non-transiting unresolved hot Jupiters with a contrast of $\sim 10^{-4}$ (Brogi et al. 2014). Lovis et al. (2016) expect a 


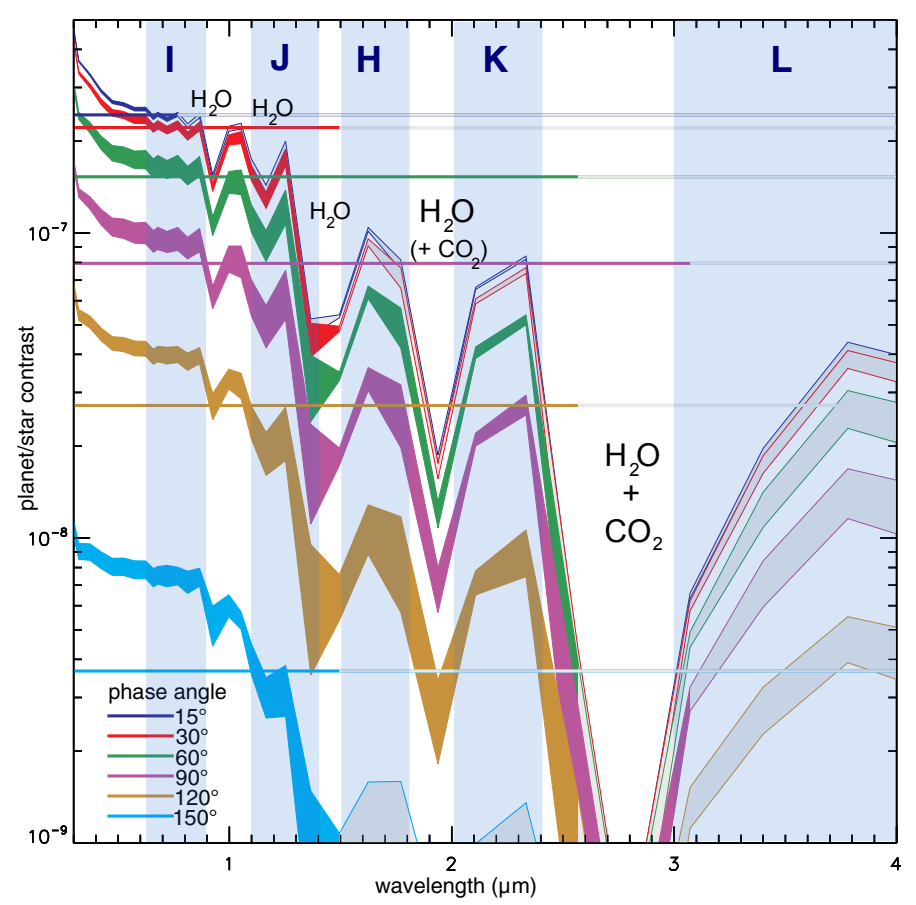

Fig. 8. Reflection spectra computed for the synchronous case with an Earth-like atmosphere. Each color corresponds to a phase angle $\left(0^{\circ}\right.$ meaning that the observer looks at the substellar point and $90^{\circ}$ at a point on the terminator). The thickness of the curve indicates the range of possible values depending on the actual observing geometry (see text and Fig. 9). Straight lines are calculated for a constant surface albedo of 0.4 . Curves are plotted in gray when the angular separation falls below twice the diffraction limit of the E-ELT $(2 \times 1.2 \lambda / D)$. These plots are obtained with a fixed planetary radius of $1.1 R_{\oplus}$. Because these plots do not include the contribution from the thermal emission, the contrast at $3.5-4 \mu \mathrm{m}$ is underestimated by a factor of $\sim 2$.

similar efficiency, which on top of the stellar extinction provided by SPHERE would allow them to reach the $\sim 10^{-7}$ contrast of Proxima $b$.

The combination of contrast and separation that is required to image Proxima $b$ should be achieved with future larger telescopes such as the E-ELT $(39 \mathrm{~m})$ or the TMT $(30 \mathrm{~m})$. For the E-ELT, 37 mas corresponds to $7 \lambda / D$ at $1 \mu \mathrm{m}$. At such angular separation, E-ELT instrumentation such as PCS (Kasper et al. 2013) is planned to achieve contrasts of $10^{-7}-10^{-8}$, a performance that is sufficient to aim to directly characterizing Proxima b.

Following Selsis et al. (2011), we can use GCM simulations to compute disk-integrated fluxes in the spectral bands of the GCM and for any observing geometry. Figures 8 and 10 present synthetic observables at visible-NIR wavelengths obtained with a simulation for an aquaplanet with an Earth-like atmosphere ( 1 bar of $\mathrm{N}_{2}, 376$ ppm of $\mathrm{CO}_{2}$, and variable $\mathrm{H}_{2} \mathrm{O}$ ) and a synchronous rotation. Figure 8 shows reflection spectra at the spectral resolution of the GCM for different phase angles and the main bands observable through Earth's atmosphere. The thickness of the curves indicates the range of contrast values that is obtained for a given phase angle, depending on the inclination of the system. For instance, both polar and equatorial observers see the planet with a $90^{\circ}$ phase angle but they do not receive the same spectral irradiance. In Fig. 8 the radius of the planet is kept constant so the contrast variation at a given wavelength is only due to the observing geometry. However, the actual mass of the planet depends on the inclination of the orbit. In Fig. 10, which

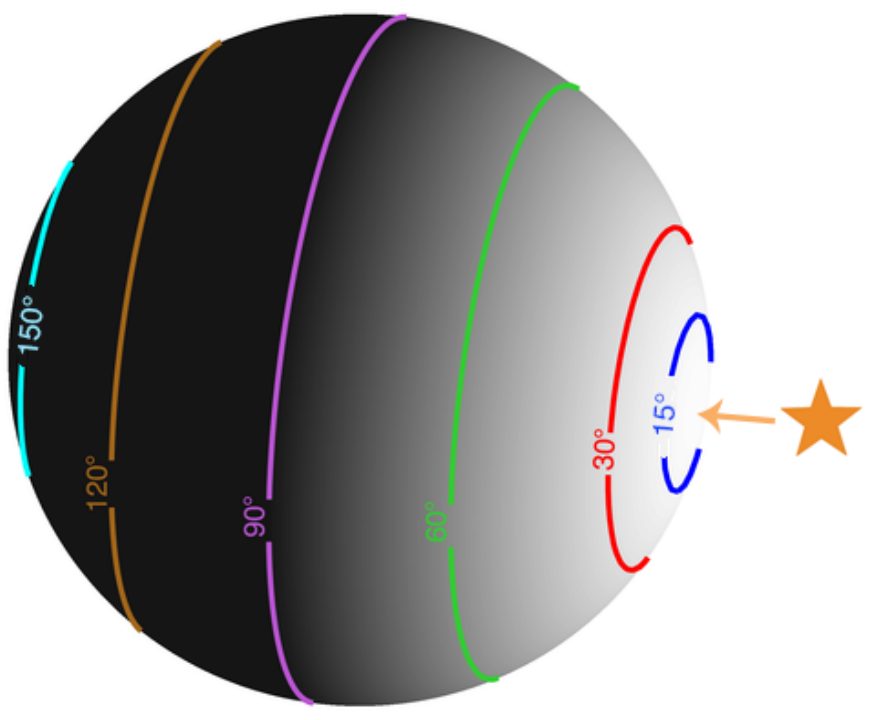

Fig. 9. Observing geometries for spectra computation. Emission and reflection spectra are presented in this article for phase angles of 15, 30, 60,90120 , and $150^{\circ}$.

shows reflection phase curves for three different inclinations and four spectral bands, we assumed the following relationship between radius and inclination of $R_{\mathrm{p}} \propto(M / \sin i)^{0.27}$.

We can see on these phase curves that low inclinations have the advantage of keeping the full orbit outside an inner working angle of twice the diffraction limit without losing contrast because of the increased planetary radius. Of course, for very low inclinations, the planet could no longer be considered "Earthlike". For instance, if $i<10^{\circ}$ (1.5\% of randomly oriented orbits) then the planet would be at least eight times more massive than the Earth. Small phase angles produce the highest contrast but imply small angular separations and short wavelengths. For phase angles smaller than $30^{\circ}$, imaging with a $39 \mathrm{~m}$ telescope is doable only in bands $I$ and $J$, but with a contrast larger than $10^{-7}$. For phase angles between 60 and $90^{\circ}$, the $H$ and $K$ bands can also be considered but with contrasts below $10^{-7}$. Imaging seems out of reach in the $L$ band $(3-4 \mu \mathrm{m})$ with a $39 \mathrm{~m}$ aperture. Considering the wavelength dependency of the contrast, the diffraction limit and the fact that adaptive optics is challenging in the visible, the $J$ band seems to represent a promising opportunity.

Sensitivity should not be an issue to directly detect Proxima b with the E-ELT. We calculated the exposure duration required to achieve a signal-to-noise ratio $(\mathrm{S} / \mathrm{N})$ of 10 per spectral channel with a spectral resolution of 100 , assuming that the angular separation would be sufficient for the noise to be dominated by the sky background (continuum + emission lines ${ }^{2}$ ) and not Speckle noise. We assumed an overall throughput of $10 \%$, integrated the background over an Airy disk and used the planetary signal derived from the GCM simulations for an inclination of $60^{\circ}$ and a phase angle of $60^{\circ}$. We obtained integration times of $5 \mathrm{~min}$ at $0.76,1.25$, and $2.3 \mu \mathrm{m}$, and $30 \mathrm{~min}$ at $1.6 \mu \mathrm{m}$.

Assuming that adaptive optics would provide sufficient efficiency to reach the required contrast and angular resolution at such short wavelengths, an $\mathrm{O}_{2}$ signature could be searched at $0.76 \mu \mathrm{m}$. This would require a high resolution to separate

\footnotetext{
2 We used the ESO documentation for the background: http://www . eso.org/sci/facilities/eelt/science/drm/ tech_data/background/
} 


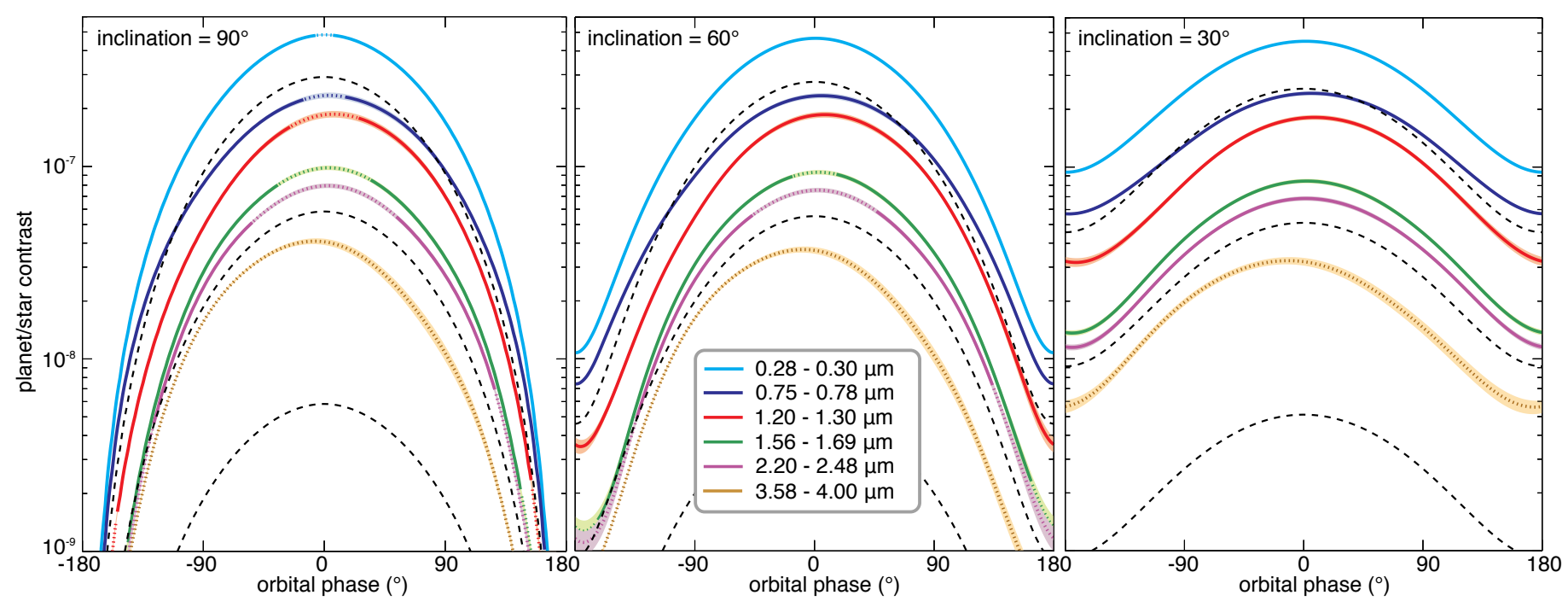

Fig. 10. Reflection phase curves computed for the synchronous case with an Earth-like atmosphere for 4 spectral bands falling into the $I, J, H$, $K$, and $L$ windows. Shadowed areas indicate the 1-sigma variability due to meteorology (mainly changing cloudiness). The dashed curves are calculated with a constant surface albedo of $0.5,0.1$, and 0.01 . Curves are dotted when the planet is inside an inner working angle of twice the diffraction limit of the E-ELT $(2 \times 1.22 \lambda / D)$. Contrary to Fig. 8, these plots include a dependency of the planetary radius on the inclination of $R \propto\left(M_{\min } / \sin i\right)^{0.27}$. We note that $60^{\circ}$ is the median value for a random distribution of inclinations.

the planet lines from the telluric lines, taking advantage of the Doppler shift of the two components. Snellen et al. (2015) suggest that a resolution of 100000 may be necessary for that, which matches that of the planned HIRES instrument ${ }^{3}$. This would imply tens of observing nights to reach a $\mathrm{S} / \mathrm{N}$ of 10 per spectral channel, but previous observations (e.g., Snellen et al. 2010, 2014; Brogi et al. 2012) have shown that a several $\sigma$ detection of a high-resolution signature by cross-correlation can be achieved with a much lower S/N per channel $(<1)$ and hence a much shorter integration time. Snellen et al. (2015) estimated that it could be doable in ten hours with the instrument IFU/E-ELT.

At $3 \mu \mathrm{m}$, the maximum angular separation of 37 mas corresponds to twice the diffraction limit of a $39 \mathrm{~m}$ aperture. Above this wavelength, imaging Proxima $b$ requires larger apertures and the Earth atmosphere (absorption and emission) becomes a major obstacle. The planet/star contrast at thermal wavelengths can, however, be orders of magnitude higher than the contrast produced by reflected wavelengths. The Earth/Sun contrast reaches $\sim 5 \times 10^{-7}$ at $10-12 \mu \mathrm{m}$, but Proxima is a star 1000 times dimmer than the Sun while its planet b could emit about the same as Earth. Contrast values of up to $\sim 5 \times 10^{-4}$ could thus be expected. In addition, thermal wavelengths provide a unique way to constrain atmospheric properties (temperature mapping at different pressure levels, day-night heat redistribution, greenhouse effect, detection of IR absorbers such as $\mathrm{H}_{2} \mathrm{O}, \mathrm{CO}_{2}, \mathrm{O}_{3}$, and $\mathrm{CH}_{4}$ ). For this reason, space telescopes using IR nulling interferometry have been considered in the past (Darwin, TPF-I) and will certainly have to be reassessed in the future as one of the main ways to characterize the atmosphere and climate of terrestial planets in nearby systems. In this context, we computed mid-IR spectra (Fig. 12) and thermal phase curves (Fig. 13) obtained with the same simulation used to produce short wavelength observables.

The phase curves we obtain are rather flat except in the 8$12 \mu \mathrm{m}$ atmospheric window where the dayside emits significantly more than the nightside. Because the flux received by the planet is rather low, the updraft of clouds and humidity on the dayside remains moderate and restricted within a small

\footnotetext{
3 Documentation for E-ELT instruments HIRES and IFU can be found at https://www.eso.org/sci/facilities/eelt/docs/
}

region around the substellar point. For this reason we do not find that most of the cooling occurs on the nightside as found by Yang et al. (2013), Gómez-Leal et al. (2012), or Bolmont et al. (2016b) for planets with an Earth-like irradiation.

Considering the history of the planet, and in particular its hot past, an Earth-like atmosphere may not be the most relevant case to address observation prospects. In Appendix B we present spectra and phase curves obtained with different compositions and rotations. As the planet could have experienced a fate similar to Venus (Ingersoll 1969), we also present reflection spectra and phase curves for a Venus-like atmosphere (Lebonnois et al. 2015), including sulphur-bearing aerosols known to produce a high albedo at visible wavelength, and then exposed to the irradiance of Proxima at the orbital distance of Proxima b.

\subsection{Prospects with James Webb Space Telescope}

Observing the modulation due to thermal phase curves does not require a transit and has been achieved by photometry in the case of both transiting and non-transiting hot Jupiters (Crossfield et al. 2010). Although very challenging, in particular because of star variability, this observation can be attempted with James Webb Space Telescope (JWST). The planet-star contrast in the mid-IR can reach $10^{-4}$ but only the amplitude of the modulation can be detected. This amplitude depends strongly on the thickness of the atmosphere as shown by the phase curves presented in Appendix B. Dense atmospheres lower the daynight temperature contrast and therefore produce rather flat light curves and modulation below $10^{-5}$ in contrast. On the other hand, planets with no or a tenuous atmosphere $(<10$ mbar) produce contrast amplitudes of $10^{-5}-10^{-4}$ in the wavelength range 8-15 $\mu \mathrm{m}$. Figure 14 shows the amplitude of the contrast modulation for a planet with no atmosphere, in radiative equilibrium, and for two inclinations. The modulation decreases with the inclination but this decrease is compensated by the increase of the mass and thus the radius. One can see that $1 \mathrm{~h}$ exposure with the JWST at $R=10$ facilitates beating the stellar photon noise above $6 \mu \mathrm{m}$. According to Belu et al. (2011), the total noise is usually within 2-3 times the stellar photon noise for wavelengths below 


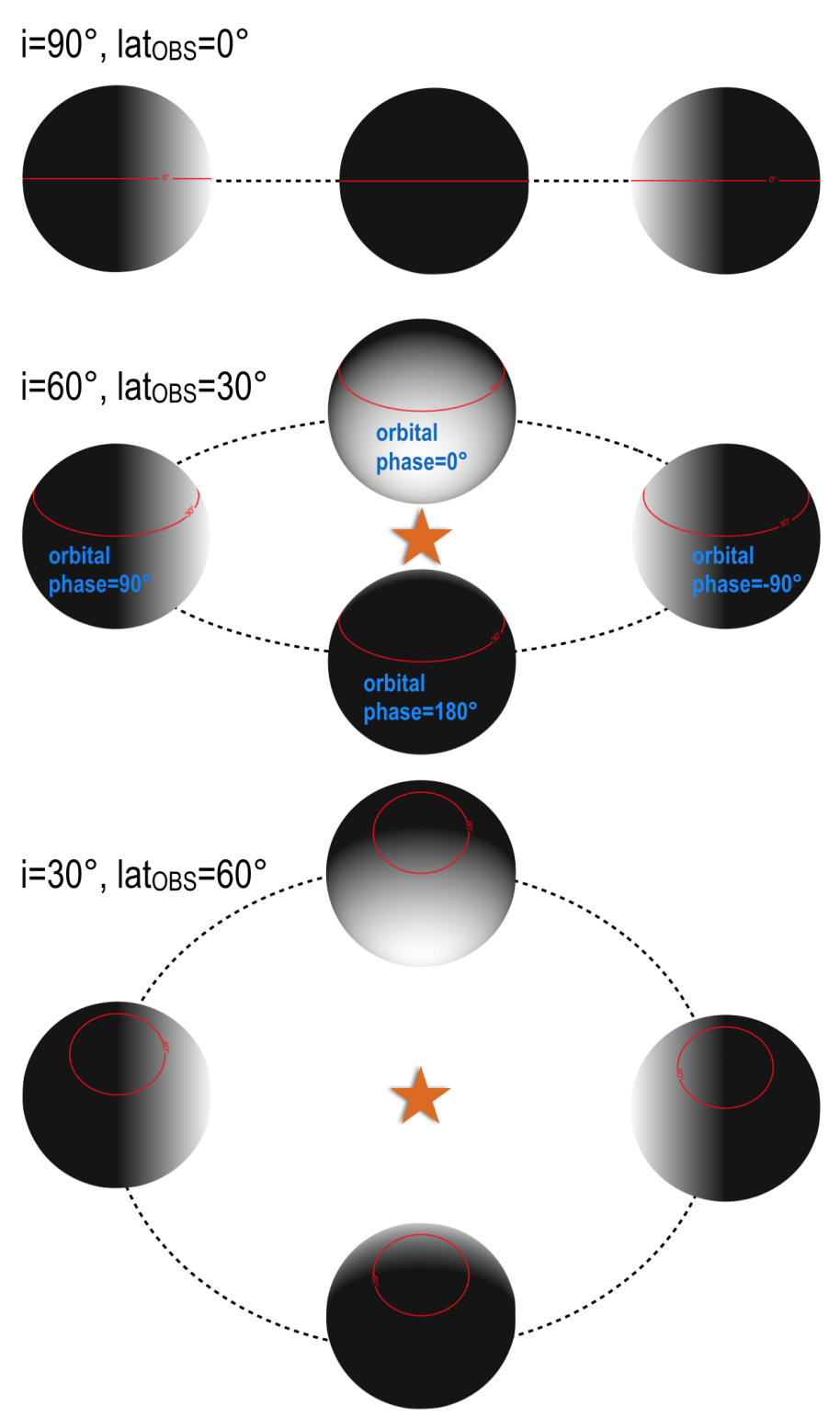

Fig. 11. Phase curves and observing geometries. We computed reflected and emitted light curves for 3 inclinations as follows: 90,60 , and $30^{\circ}$ corresponding to subobserver latitude of 0,30 , and $60^{\circ}$, respectively (as the we assumed a null obliquity). The subobserver latitude is indicated with a red line. In Appendix B, phase curves are shown only for an inclination of $60^{\circ}$ (the median value for randomly oriented orbits).

$15 \mu \mathrm{m}$; above this limit the thermal emission from the telescope dramatically degrades the observations. Detecting these modulations with JWST would be extremely challenging because of stellar variability and flares. But flux variations are smaller in the infrared and the orbital period and ephemeris of the planet are known, which considerably helps planning short exposure over several orbits, in particular near the peak at superior conjunction. Measuring a modulation would point to planets with no dense atmosphere such as Mercury or Mars. In theory, measurement at different wavelengths could be used to find atmospheric signatures (Selsis et al. 2011), constrain the radius, albedo, and inclination of the planet (Maurin et al. 2012) as well as its rotation (Selsis et al. 2013).

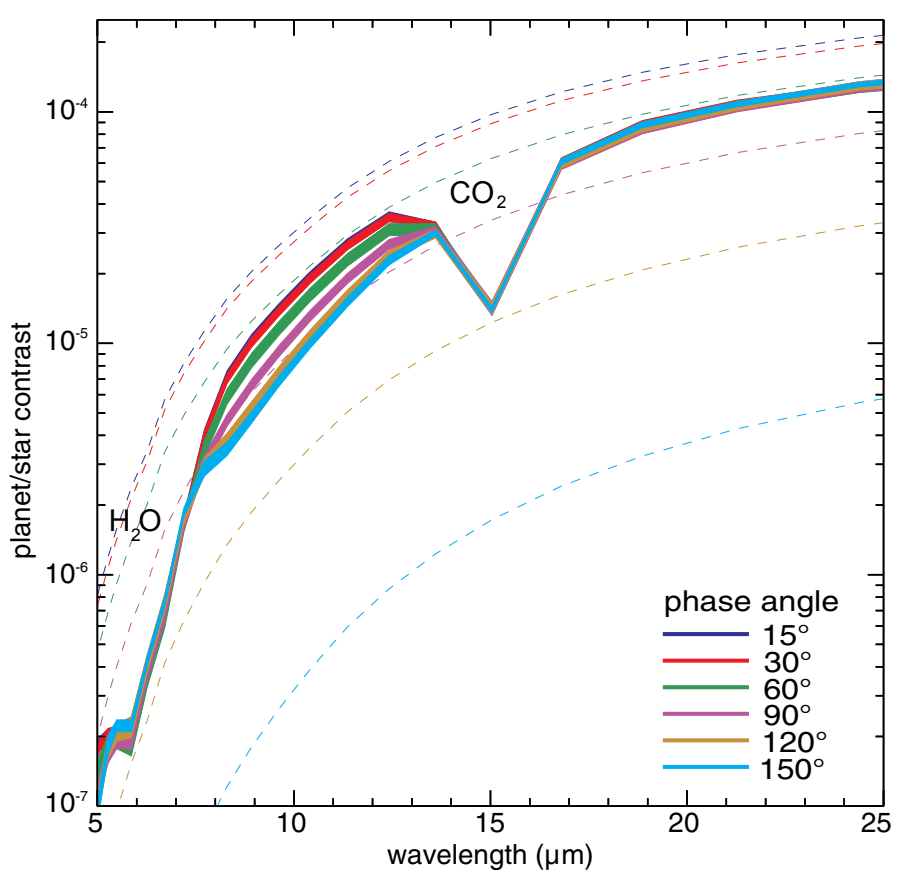

Fig. 12. Emission spectra computed for the synchronous case with an Earth-like atmosphere. Each color corresponds to a phase angle. The thickness of the curves indicate the variability associated with inclination. Dashed lines are calculated for a planet with no atmosphere with a constant surface albedo of 0.2 . These plots are obtained with a fixed planetary radius of $1.1 R_{\oplus}$, whatever the inclination of the orbit.

\section{Discussions}

The modeling work performed here to explore the possible climates and observability of Proxima b remains speculative. Major surprises in the composition of the atmosphere or the nature of the planet cannot be discarded. Within the known uncertainties, we can list the following points:

1. The luminosity of Proxima Centauri is not perfectly known: using interferometry with two VLT telescopes, Demory et al. (2009) measured the radius of Proximab and its effective temperature and found $R=0.141 \pm 0.007 R_{\odot}$ and $T_{\text {eff }}=3098 \pm 56 \mathrm{~K}$, which yields a bolometric luminosity of $0.00165 \pm 0.00012 L_{\odot}$. In this study we used a value of $0.0017 L_{\odot}$ while Anglada-Escudé et al. (2016) give $0.00155 L_{\odot}$ as a median value that is derived from Boyajian et al. (2012). Both values are within the uncertainty of Demory et al. (2009) and Boyajian et al. (2012). Although changing the actual bolometric flux received by Proxima $b$ would slightly alter surface and atmosphere temperatures found for a given atmospheric composition, this discrepancy does qualitatively not impact our results. Changing the actual bolometric flux received by Proxima $b$ would of course alter the detailed relationship between atmospheric pressure and temperature. Considering the importance of this system, the community should agree on a standard and calibrated irradiance spectrum of Proxima to be used for climate and habitability studies.

2. The amount of background gas: some simulations in this work included background $\mathrm{N}_{2}$ while others did not. It would require dedicated absorption coefficients to perform all the GCM simulations with a fixed $\mathrm{N}_{2}$ partial pressure. Moreover, we have bad constraints on the amount of background gas available on the planet because of, first, uncertainties on the mass of Proxima b and, second, the possibility that the 
M. Turbet et al.: The habitability of Proxima Centauri b. II.

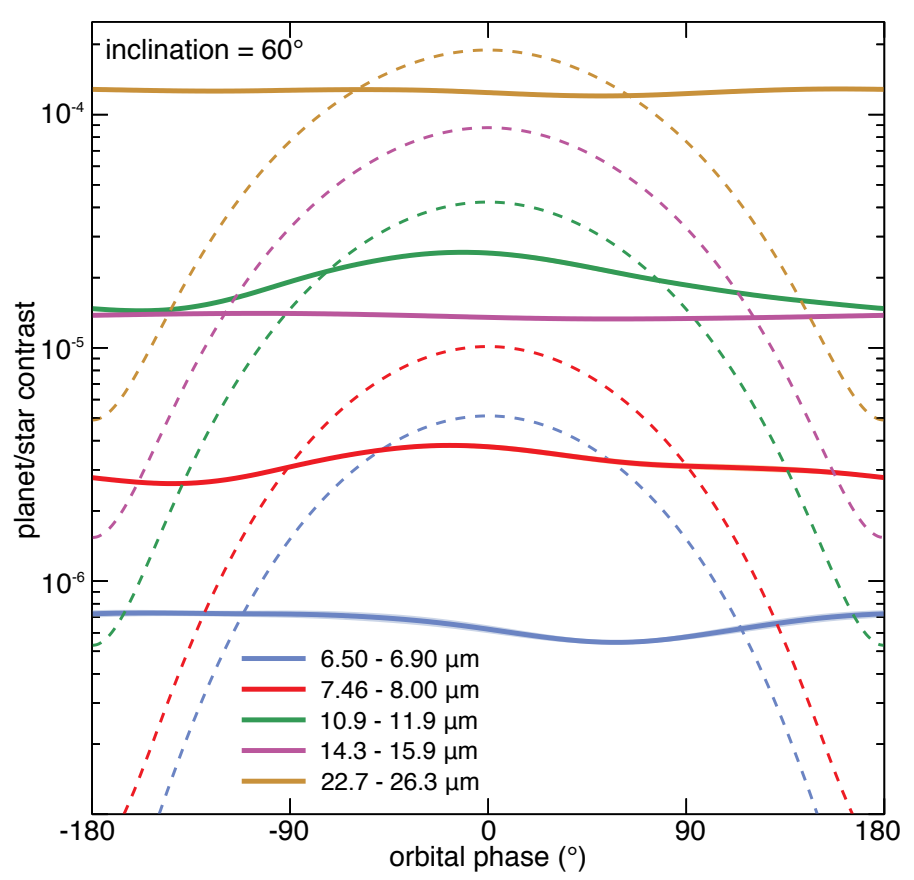

Fig. 13. Emission phase curves computed for the synchronous case with an Earth-like atmosphere, for 4 spectral bands used in the GCM. Gray areas indicate the 1-sigma variability due to meteorology (mainly changing cloudiness). Dashed curves are calculated for a planet with no atmosphere with a constant surface albedo of 0.2. Contrary to Fig. 12, these plots include a dependency of the planetary radius on the inclination of $R \propto(M / \sin i)^{0.27}$.

background gas was lost to space as a result of the high XUV flux of Proxima Centauri (Ribas et al. 2016).

3 . The convection scheme: it should be kept in mind that models like Yang et al. (2013) find higher albedos in the substellar area owing to a different convection scheme and possibly different cloud parametrizations. As Proxima $b$ is moderately irradiated, this discrepancy might not be as important as for the near-inner edge cases studied by Yang et al. (2013).

4. The oceanic circulation: we recall that our results in the aquaplanet regime neglected the effect of oceanic transport. This is an important era of future improvement, although it adds several new unconstrained ingredients, such as the presence and location of continents.

5. Climate retroactions: we assumed in this study that the amount of volatiles $\left(\mathrm{H}_{2} \mathrm{O}, \mathrm{CO}_{2}\right.$, and $\left.\mathrm{N}_{2}\right)$ should be uncorrelated and therefore that each configuration of volatile inventory could be achievable by Proxima b. In fact, climate retroactions such as the carbonate-silicate cycle (Walker et al. 1981) could favor some of these configurations by linking the amount of carbon dioxide and water.

\section{Conclusions}

In this study, we explored the possible climates of Proxima Centauri $b$ for a wide range of volatile inventories of water, carbon dioxide, and nitrogen. It appears from our results that the habitability of the planet is possible for a very broad range of atmospheric pressures and compositions, as shown by the size of the blue regions in Fig. 1.

In a nutshell, the presence of surface liquid water requires either a large surface inventory of water (a global ocean able to resupply $\mathrm{H}_{2} \mathrm{O}$ to the dayside by deep circulation) or an atmosphere

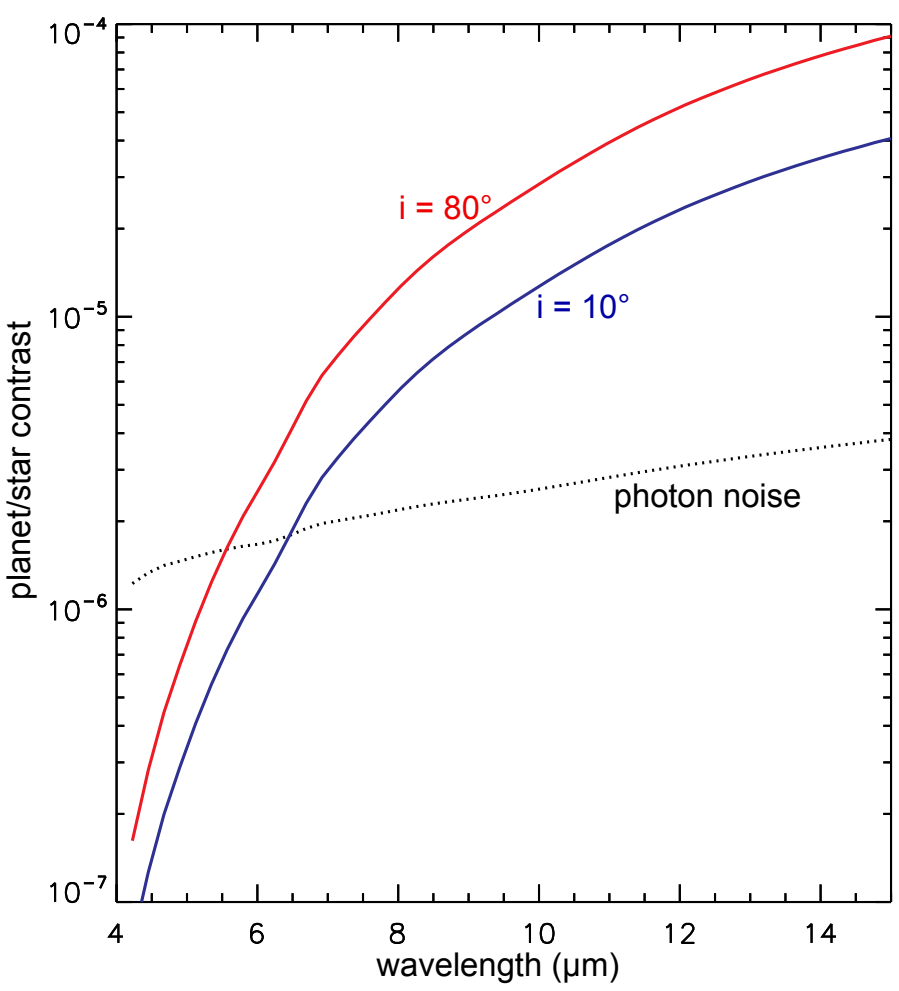

Fig. 14. Observability of the photometric modulation due to thermal phase curves with JWST. The amplitude of the modulation is calculated for a planet with no atmosphere and a Bond albedo of 0.2 and with a radius that scales with the inclination as $R \propto(M / \sin i)^{0.27}$. The noise is computed for a $1 \mathrm{~h}$ exposure, a spectral resolution of 10 , and the collecting area of the JWST $\left(25 \mathrm{~m}^{2}\right)$.

with a strong enough greenhouse effect that increases surface temperatures above the freezing point of water everywhere.

Apart from receiving the necessary insolation, this study tells us that the key ingredient to the habitability of a planet is the retention of all volatiles, such as water, of course, but also noncondensible gases (with a greenhouse effect or not) to warm surface cold traps. Ribas et al. (2016) showed that it is possible that the planet lost large amounts of water. However, this work shows that even with extremely low amounts of water (a few $10^{-3}$ Earth ocean content for the synchronous case and less than $10^{-5}$ for the asynchronous case), there are $\mathrm{CO}_{2}$ pressures that allow surface liquid water.

More generally, these conclusions are not restricted to the case of Proxima b. In fact, any low-obliquity planet within the classical habitable zone of its star should be in one of the climate regimes discussed here, although the limits between the various regimes would shift quantitatively with the planet parameters (e.g., the insolation).

Prospects for direct imaging with E-ELT are extremely promising: the star-planet separation reaches $9.5 \lambda / D$ at $760 \mathrm{~nm}$ (wavelength of the $\mathrm{O}_{2}$ band) with a contrast of $0.9-5 \times 10^{-7}$ (depending on atmospheric and surface composition, and planetary radius) and $3.6 \lambda / D$ at $2 \mu \mathrm{m}$ with a contrast of $10^{-7}-10^{-8} . J$ band (1.1-1.4 $\mu \mathrm{m})$ offers a fine trade-off in terms of separation, contrast, and constraints on adaptive optics. The brightness of the planet should allow high-resolution spectroscopy and the search for a variety of molecular signatures, including $\mathrm{O}_{2}, \mathrm{H}_{2} \mathrm{O}, \mathrm{CO}_{2}$, and $\mathrm{CH}_{4}$.

Thermal phase curve modulations are observable - in theory - with JWST, with a contrast of $\sim 10^{-5}$ at $10 \mu \mathrm{m}$ but will be challenging because of stellar variability. More accurate mid-IR 
spectroscopy would probably require space-based interferometry. Most of our knowledge on planetary atmospheres and habitability come from the study of Venus, Mars, and Earth. Proxima $b$ could potentially be the fourth terrestrial planet to confront all that we know on these domains.

Acknowledgements. M.T. thanks Sébastien Lebonnois for his help on the modeling of a venusian atmosphere with the LMD Generic Model. E.B. acknowledges that this work is part of the F.R.S.-FNRS "ExtraOrDynHa" research project. I.R acknowledges support from the Spanish Ministry of Economy and Competitiveness (MINECO) through grant ESP2014-57495-C2-2-R. S.N.R. acknowledges support from the Agence Nationale de la Recherche via grant ANR-13-BS050003-002 (project MOJO).

\section{References}

Abbot, D. S., \& Switzer, E. R. 2011, ApJ, 735, L27

Abe, Y., Abe-Ouchi, A., Sleep, N. H., \& Zahnle, K. J. 2011, Astrobiology, 11 443

Anglada-Escudé, G., Amado, P. J., Barnes, J., et al. 2016, Nature, in press

Bazot, M., Christensen-Dalsgaard, J., Gizon, L., \& Benomar, O. 2016, MNRAS, 460, 1254

Belu, A. R., Selsis, F., Morales, J.-C., et al. 2011, A\&A, 525, A83

Bézard, B., Tsang, C. C. C., Carlson, R. W., et al. 2009, J. Geophys. Res. Planets, 114, E00B39

Bolmont, E., Libert, A.-S., Leconte, J., \& Selsis, F. 2016a, A\&A, 591, A106

Bolmont, E., Selsis, F., Owen, J. E., et al. 2016b, MNRAS, 46, 3728

Bougher, S. W., Hunten, D. M., \& Phillips, R. J. 1997, Venus II, Vol. 165

Boyajian, T. S., von Braun, K., van Belle, G., et al. 2012, ApJ, 757, 112

Brogi, M., Snellen, I. A. G., de Kok, R. J., et al. 2012, Nature, 486, 502

Brogi, M., de Kok, R. J., Birkby, J. L., Schwarz, H., \& Snellen, I. A. G. 2014 A\&A, 565, A124

Charnay, B., Forget, F., Wordsworth, R., et al. 2013, J. Geophys. Res. Atmospheres, 118, 414

Crossfield, I. J. M., Hansen, B. M. S., Harrington, J., et al. 2010, ApJ, 723, 1436

Davies, J. H., \& Davies, D. R. 2010, Solid Earth, 1, 5

Demory, B.-O., Ségransan, D., Forveille, T., et al. 2009, A\&A, 505, 205

Eymet, V., Coustet, C., \& Piaud, B. 2016, J. Phys. Conf. Ser., 676, 012005

Forget, F. 2013, Int. J. Astrobiol., 12, 177

Forget, F., \& Pierrehumbert, R. T. 1997, Science, 278, 1273

Forget, F., Wordsworth, R., Millour, E., et al. 2013, Icarus, 222, 81

Fu, Q., \& Liou, K. N. 1992, J. Atmos. Sci., 49, 2139

Gómez-Leal, I., Pallé, E., \& Selsis, F. 2012, ApJ, 752, 28

Hourdin, F., Musat, I., Bony, S., et al. 2006, Climate Dynamics, 27, 787

Hu, Y., \& Yang, J. 2014, Proceedings of the National Academy of Science, 111, 629

Ingersoll, A. P. 1969, J. Atmos. Sci., 26, 1191

Joshi, M. M., \& Haberle, R. M. 2012, Astrobiology, 12, 3

Kasper, M., Verinaud, C., \& Mawet, D. 2013, in Proc. Third AO4ELT Conference, eds. S. Esposito, \& L. Fini, 8

Kaspi, Y., \& Showman, A. P. 2015, ApJ, 804, 60

Kasting, J. F., Whitmire, D. P., \& Reynolds, R. T. 1993, Icarus, 101, 108

Kitzmann, D. 2016, ApJ, 817, L18
Kopparapu, R. K., Ramirez, R., Kasting, J. F., et al. 2013, ApJ, 765, 131 Kopparapu, R. K., Ramirez, R. M., SchottelKotte, J., et al. 2014, ApJ, 787, L29 Kopparapu, R. K., Wolf, E. T., Haqq-Misra, J., et al. 2016, ApJ, 819, 84 Lammer, H., Bredehöft, J. H., Coustenis, A., et al. 2009, A\&ARv, 17, 181

Lawson, P. R., Poyneer, L., Barrett, H., et al. 2012, in Adaptive Optics Systems III, Proc. SPIE, 8447, 844722

Lebonnois, S., Eymet, V., Lee, C., \& Vatant d'Ollone, J. 2015, J. Geophys. Res. Planets, 120, 1186

Leconte, J., Forget, F., Charnay, B., Wordsworth, R., \& Pottier, A. 2013a, Nature, 504, 268

Leconte, J., Forget, F., Charnay, B., et al. 2013b, A\&A, 554, A69

Lovis, C., Snellen, I., Mouillet, D., et al. 2016, A\&A, submitted [arXiv: 1609.03082]

Maurin, A. S., Selsis, F., Hersant, F., \& Belu, A. 2012, A\&A, 538, A95

Menou, K. 2013, ApJ, 774

Millour, E., Forget, F., Spiga, A., et al. 2015, European Planetary Science Congress 2015, 10, EPSC2015-438

Moore, J. M., McKinnon, W. B., Spencer, J. R., et al. 2016, Science, 351, 1284

Petrenko, V. F., \& Whitworth, R. W. 2002, in Physics of Ice (New York, Oxford University Press)

Pierrehumbert, R. T. 2011, ApJ, 726, L8

Rajpurohit, A. S., Reylé, C., Allard, F., et al. 2013, A\&A, 556, A15

Read, P., \& Lewis, S. R. 2004, in The Martian Climate Revisited (Springer)

Ribas, I., Bolmont, E., Selsis, F., et al. 2016, A\&A, 596, A111

Richard, C., Gordon, I. E., Rothman, L. S., et al. 2012, J. Quant. Spectr. Rad. Transf., 113, 1276

Rothman, L. S., Gordon, I. E., Barbe, A., et al. 2009, J. Quant. Spectr. Rad. Transf., 110, 533

Selsis, F., Wordsworth, R. D., \& Forget, F. 2011, A\&A, 532, A1

Selsis, F., Maurin, A.-S., Hersant, F., et al. 2013, A\&A, 555, A51

Shields, A. L., Bitz, C. M., Meadows, V. S., Joshi, M. M., \& Robinson, T. D. 2014, ApJ, 785, L9

Showman, A. P., \& Polvani, L. M. 2011, ApJ, 738, 71

Snellen, I. A. G., de Kok, R. J., de Mooij, E. J. W., \& Albrecht, S. 2010, Nature, 465, 1049

Snellen, I. A. G., Brandl, B. R., de Kok, R. J., et al. 2014, Nature, 509, 63

Snellen, I., de Kok, R., Birkby, J. L., et al. 2015, A\&A, 576, A59

Spencer, J. R., Rathbun, J. A., Travis, L. D., et al. 2000, Science, 288, 1198

Stofan, E. R., Elachi, C., Lunine, J. I., et al. 2007, Nature, 445, 61

Turbet, M., Forget, F., Head, J., \& Wordsworth, R. 2016, Icarus, submitted

Van Leeuwen, F. 2007, A\&A, 474, 653

Wagner, W., Saul, A., \& Pruss, A. 1994, J. Phys. Chem. Ref. Data, 23, 515

Walker, J. C. G., Hays, P. B., \& Kasting, J. F. 1981, J. Geophys. Res., 86, 9776

Warren, S. G. 1984, Annals of Glaciology, 5, 177

Warren, S. G., \& Wiscombe, W. J. 1980, J. Atm. Sci., 37, 2734

Wordsworth, R. D., \& Pierrehumbert, R. T. 2013, ApJ, 778, 154

Wordsworth, R., Forget, F., \& Eymet, V. 2010, Icarus, 210, 992

Wordsworth, R. D., Forget, F., Selsis, F., et al. 2011, ApJ, 733, L48

Wordsworth, R., Forget, F., Millour, E., et al. 2013, Icarus, 222, 1

Wordsworth, R. D., Kerber, L., Pierrehumbert, R. T., Forget, F., \& Head, J. W. 2015, J. Geophys. Res. Planets, 120, 1201

Yang, J., Cowan, N. B., \& Abbot, D. S. 2013, ApJ, 771, L45

Yang, J., Liu, Y., Hu, Y., \& Abbot, D. S. 2014, ApJ, 796, L22

Zuber, M. T., Smith, D. E., Lemoine, F. G., \& Neumann, G. A. 1994, Science, 266, 1839 


\section{Appendix A: Computation of maximal ice thickness before basal melting}

Because the ice thermal conductivity can vary substantially with temperature according to the relation $\lambda_{\text {ice }}(T)=A / T$ with $A=$ $651 \mathrm{~W} \mathrm{~m}^{-1}$ (Petrenko \& Whitworth 2002), the temperature profile inside an ice layer in equilibrium follows an exponential law from which we derive a maximum thickness $h_{\text {ice }}^{\max }$ before melting (Abbot \& Switzer 2011),

$h_{\text {ice }}^{\max }=\frac{A}{F_{\text {geo }}} \ln \left(\frac{T_{\text {melt }}}{T_{\text {surf }}}\right)$,

where $F_{\text {geo }}$ is the internal heat flux and $T_{\text {melt }}$ is the melting temperature of ice at the base of the glacier. For pressure lower than 100 bars (ice thickness lower than $\sim 1 \mathrm{~km}$ ), $T_{\text {melt }}$ is roughly constant and equal to $273 \mathrm{~K}$. However, this assumption does not work for higher pressures. Thus, we use the following parametrization for the melting curve of ice (Wagner et al. 1994):

$$
\begin{aligned}
P_{\text {melt }}(T)= & P_{\text {ref }}\left[1-0.626 \times 10^{6}\left(1-\left(\frac{T}{T_{\text {ref }}}\right)^{-3}\right)\right. \\
& \left.+0.197135 \times 10^{6}\left(1-\left(\frac{T}{T_{\text {ref }}}\right)^{21.2}\right)\right],
\end{aligned}
$$

where $T_{\text {ref }}$ and $P_{\text {ref }}$ are the temperature and pressure of the triple point of water.

Using the relation $h_{\text {ice }}^{\text {max }}=\frac{P_{\text {melt }}-P_{\text {surf }}}{\rho_{\text {ice }} g}$ and following a similar approach to Leconte et al. (2013b), we can solve the system of Eqs. (A.1), (A.2) implicitly (and numerically) and find the thickness at which melting occurs at the base of the glaciers.

\section{Appendix B: Spectra and phase curves}

We present here synthetic spectra and phase curves obtained with some of the GCM simulations. Reflected and emitted spectra are obtained as in Figs. 8 and 12, respectively. Reflection and thermal phase curves are computed as in Figs. 10 and 13, respectively, for a $60^{\circ}$ inclination and a radius $R=\left(M_{\min } / \sin 60^{\circ}\right)^{0.27}=$ $1.11 R_{\oplus}$. Color codes for phase angles and wavelengths are the same as for figures in the main text.

To describe each different type of observables (reflected spectra, reflected lightcurves, thermal spectra, and thermal lightcurves), we start from dry cases with tenuous atmosphere, which exhibit the most simple features, and progress toward dense and humid atmospheres that combine more effects, including clouds.

\section{B.1. Reflected spectra}

Reflected spectra are shaped by Rayleigh scattering from the gas, Mie scattering from clouds, molecular absorption features, and surface reflectivity.

- Dry case, Earth-like atmosphere (Fig. B.3): we can see the decrease of the albedo with increasing wavelengths in the UV-visible domain due to the combination of Rayleigh scattering and the constant surface albedo of 0.2. $\mathrm{CO}_{2}$ absorption features can be seen at 1.9 and 2.6-2.7 $\mu \mathrm{m}$. The rotation mode of the planet does not affect the observables.

- Dry case, 1 bar of $\mathrm{CO}_{2}$ (Fig. B.4): same as above with more and deeper $\mathrm{CO}_{2}$ features.
- Aquaplanet, mainly frozen, 10 mbar of $\mathrm{N}_{2} ; 376$ ppm of $\mathrm{CO}_{2}$ (Fig. B.5): Rayleigh scattering is negligible and the only atmospheric features are the $\mathrm{H}_{2} \mathrm{O}$ bands. The drop of albedo between 1 and $1.5 \mu \mathrm{m}$ is produced by the wavelengthdependent reflectivity of ice included in the model (Fig. 2). This drop is attenuated in the synchronized case because the dayside is partly covered by liquid water (Fig. 6) whose reflectivity is constant with wavelength $(\sim 7 \%)$ and has a value close to that of ice in the infrared $(\sim 5 \%)$. For this reason, the overall albedo is also higher for the 3:2 rotation, the surface being mostly covered by ice.

- Aquaplanet, mainly frozen, 1 bar of $\mathrm{N}_{2} ; 376 \mathrm{ppm}$ of $\mathrm{CO}_{2}$ (Fig. B.6): in addition to the features described in the previous case, this configuration also exhibits signatures of the larger water vapor content due to higher temperatures: deeper $\mathrm{H}_{2} \mathrm{O}$ absorption bands and a larger spatial and temporal variability due to meteorology. In synchronous rotation, the Rayleigh slope can be seen but not in the nonsynchronous case because the albedo of the icy surface dominates over the albedo due to Rayleigh scattering.

- Aquaplanet, 1 bar of $\mathrm{CO}_{2}$ (Fig. B.7): this case exhibits the strongest molecular absorptions due to large columns of both $\mathrm{CO}_{2}$ and water vapor. The low albedo of the liquid water surface reveals the atmospheric Rayleigh slope at wavelength lower than $600 \mathrm{~nm}$. The spectrum of the synchronous case is very sensitive to the observing geometry for a given phase angle. As seen in Fig. B.2, this is due to the concentration of clouds at low latitude and eastward of the substellar point in the synchronous case, while the 3:2 case has very uniform cloud coverage.

\section{B.2. Reflected phase curves}

Departures between the observed phase curve and phase curve produced by a sphere with uniform surface albedo are due to longitudinal variations of reflectivity on the dayside, which, in our cases, can be due to cloud coverage or change in the nature of the surface (liquid versus icy).

- Dry case, Earth-like atmosphere (Fig. B.9): the lightcurves are those expected for a sphere with a uniform albedo. As the atmosphere is very transparent at visible-NIR wavelengths, the value of this albedo is 0.2 (the wavelength-independent value attributed to the dry surface in the model) for all the bands except the one in the UV (cyan) that exhibits a higher albedo from Rayleigh scattering.

- Dry case, 1 bar of $\mathrm{CO}_{2}$ (Fig. B.10): same as above, except for the 1.6 (green) and $1.25 \mu \mathrm{m}$ (red) bands that are attenuated by $\mathrm{CO}_{2}$ absorption.

- Aquaplanet, mainly frozen, 10 mbar of $\mathrm{N}_{2} ; 376$ ppm of $\mathrm{CO}_{2}$ (Fig. B.11): we note again the overall decrease of albedo with increasing wavelength (a property of ice). While the phase curves of the asynchronous case present no longitudinal change of reflectivity, the phase curves of the synchronous case show a flattening at opposition at wavelength below $1.3 \mu \mathrm{m}$, which is due to the different albedo of ice and liquid water. This decrease in albedo at small phase angles is therefore a signature of the "eye ball" configuration that is observable here because the atmosphere and cloud cover are thin enough to give access to the surface reflectivity.

- Aquaplanet, mainly frozen, 1 bar of $\mathrm{N}_{2} ; 376 \mathrm{ppm}$ of $\mathrm{CO}_{2}$ (Fig. B.12): the "eye ball" signature of the synchronous case is still noticeable at wavelengths not absorbed by water vapor and not dominated by Rayleigh scattering, so typically 

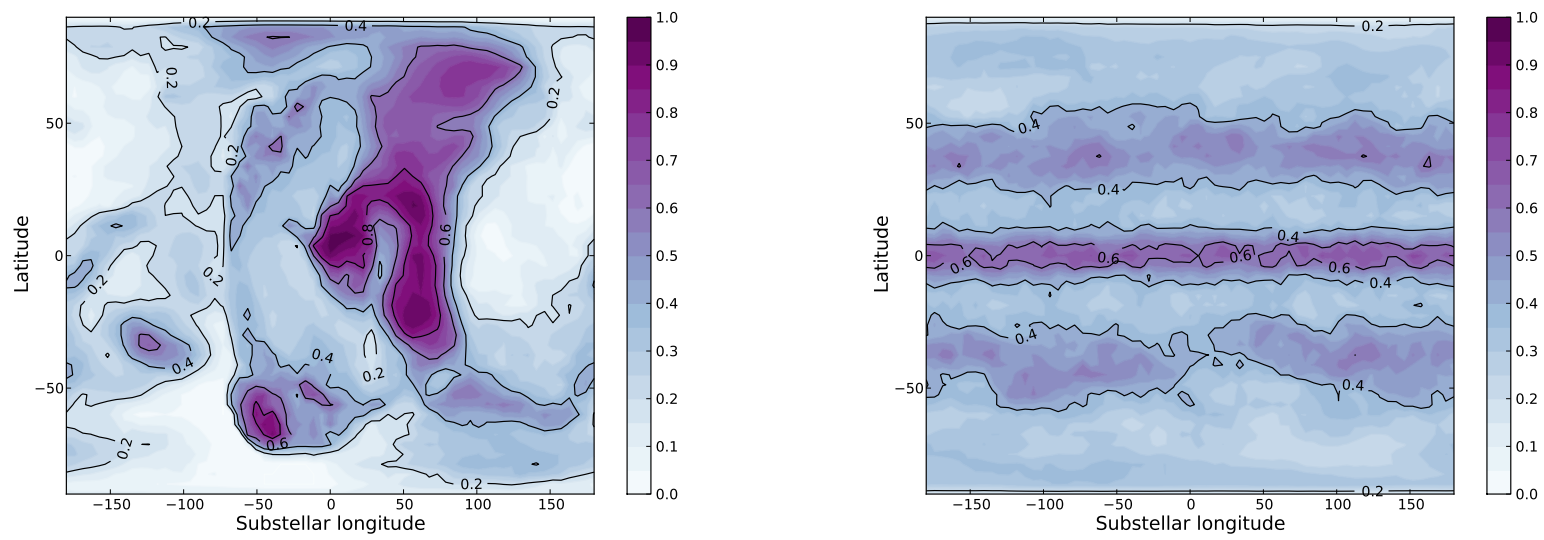

Fig. B.1. Cloud maps for an aquaplanet with an Earth-like atmospheric composition ( 1 bar of $\mathrm{N}_{2}, 376 \mathrm{ppm}$ of $\left.\mathrm{CO}_{2}\right)$. Left: synchronous rotation. Right: 3:2 spin-orbit resonance. In both cases, longitude is given relative to the substellar point. Colors indicate the fractional cloud cover, averaged over 2 orbits.
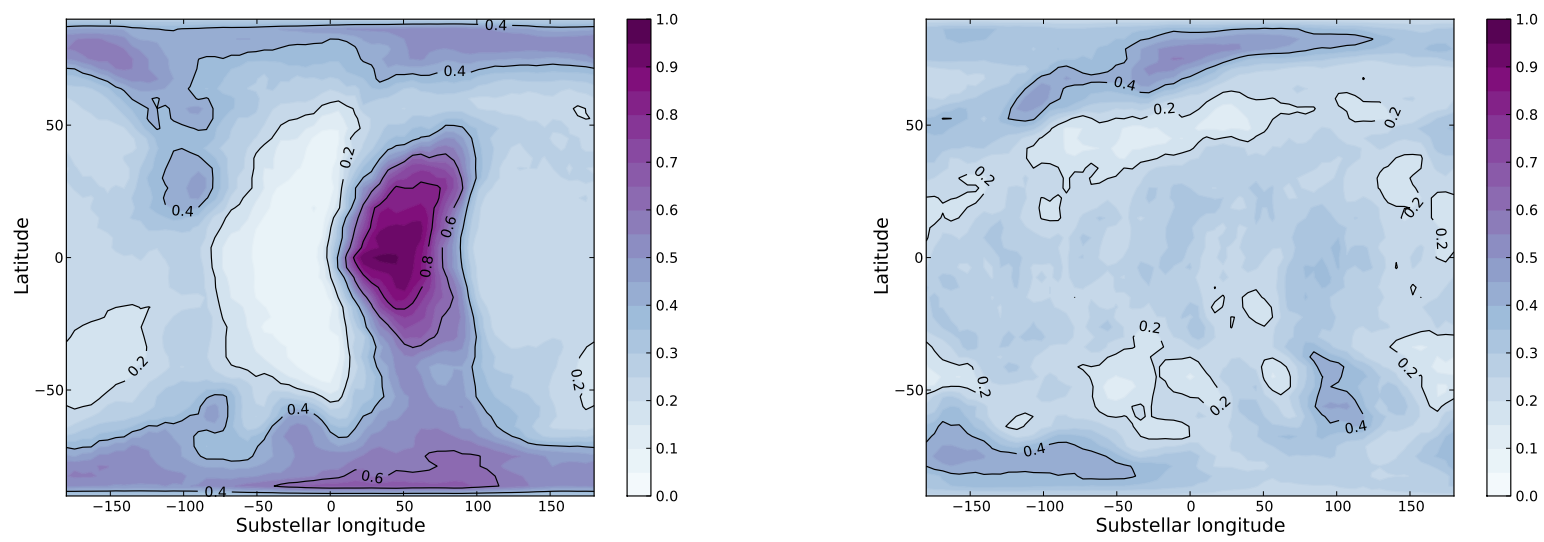

Fig. B.2. Cloud maps for an aquaplanet with a 1 bar $\mathrm{CO}_{2}$ atmosphere. Left: synchronous rotation. Right: 3:2 spin-orbit resonance. In both cases, longitude is given relative to the substellar point. Colors indicate the fractional cloud cover, averaged over 2 orbits.

between 0.5 and $0.8 \mu \mathrm{m}$. Clouds reduce the signature without hiding it completely as they cover the dayside ocean (see Fig. B.1) only partially and because their albedo is still lower than that of ice at these wavelengths. Because thick clouds tend to accumulate eastward of the substellar point, the phase curves are asymmetric except at UV wavelengths that are backscattered above the clouds. In the asynchronous case clouds are uniformly distributed in longitude and do not produce significant asymmetry.

- Aquaplanet, 1 bar of $\mathrm{CO}_{2}$ (Fig. B.13): both the synchronous and asynchronous cases exhibit a high variability due to meteorology, which is a result of high temperatures and strong water cycle. The visible and NIR phase curves of the synchronous case are very asymmetric. The reason is the same as in the previous case but with a sharper transition in cloudiness with a clear sky west of the substellar point and a dense cloud patch east of the substellar point, as seen in Fig. B.2.

\section{B.3. Emission spectra}

Thermal emission spectra are influenced by the temperature distribution at the surface at wavelengths where the atmosphere is transparent and by the thermal structure of the atmosphere at other wavelengths. In theory, emission spectra are also shaped by the surface emissivity but in our model it is fixed to unity in most cases.
- Dry case, Earth-like atmosphere (Fig. B.15): in the synchronous case, the spectra are similar to those of sphere in radiative equilibrium except in the $\mathrm{CO}_{2}$ band, which is emitted by a horizontally uniform layer of the upper atmosphere. At high phase angle and long wavelengths, differences from the sphere in radiative equilibrium appear owing to the transport of heat toward the nightside that contributes to the emission. In 3:2 rotation, the spectra are similar but more sensitive to the observing geometry at a given phase angle as the surface temperature map is no longer symmetric relative to the substellar point.

- Dry case, 1 bar of $\mathrm{CO}_{2}$ (Fig. B.16): in addition to the $15 \mu \mathrm{m}$ band, high pressure $\mathrm{CO}_{2}$ features including the hot bands at 9.5 and $11 \mu \mathrm{m}$ and a CIA feature between 6.5 and $8 \mu \mathrm{m}$. These absorption features probe uniform high-altitude atmospheric layers and do not depend on the subobserver position, while windows probe the surface and exhibit a dependency on the observing geometry (synchronous) and variability (3:2).

- Aquaplanet, mainly frozen, 10 mbar of $\mathrm{N}_{2} ; 376$ ppm of $\mathrm{CO}_{2}$ (Fig. B.17): the spectra are featureless except for a shallow $\mathrm{H}_{2} \mathrm{O}$ signature at 6-7 $\mu \mathrm{m}$ in the synchronous case. They depart from the spectra of a sphere in radiative equilibrium, in particular at long wavelengths, as the surface temperature does not drop below $200 \mathrm{~K}$ (synchronous) and $150 \mathrm{~K}$ (3:2) thanks to a redistribution of latent heat. 
M. Turbet et al.: The habitability of Proxima Centauri b. II.
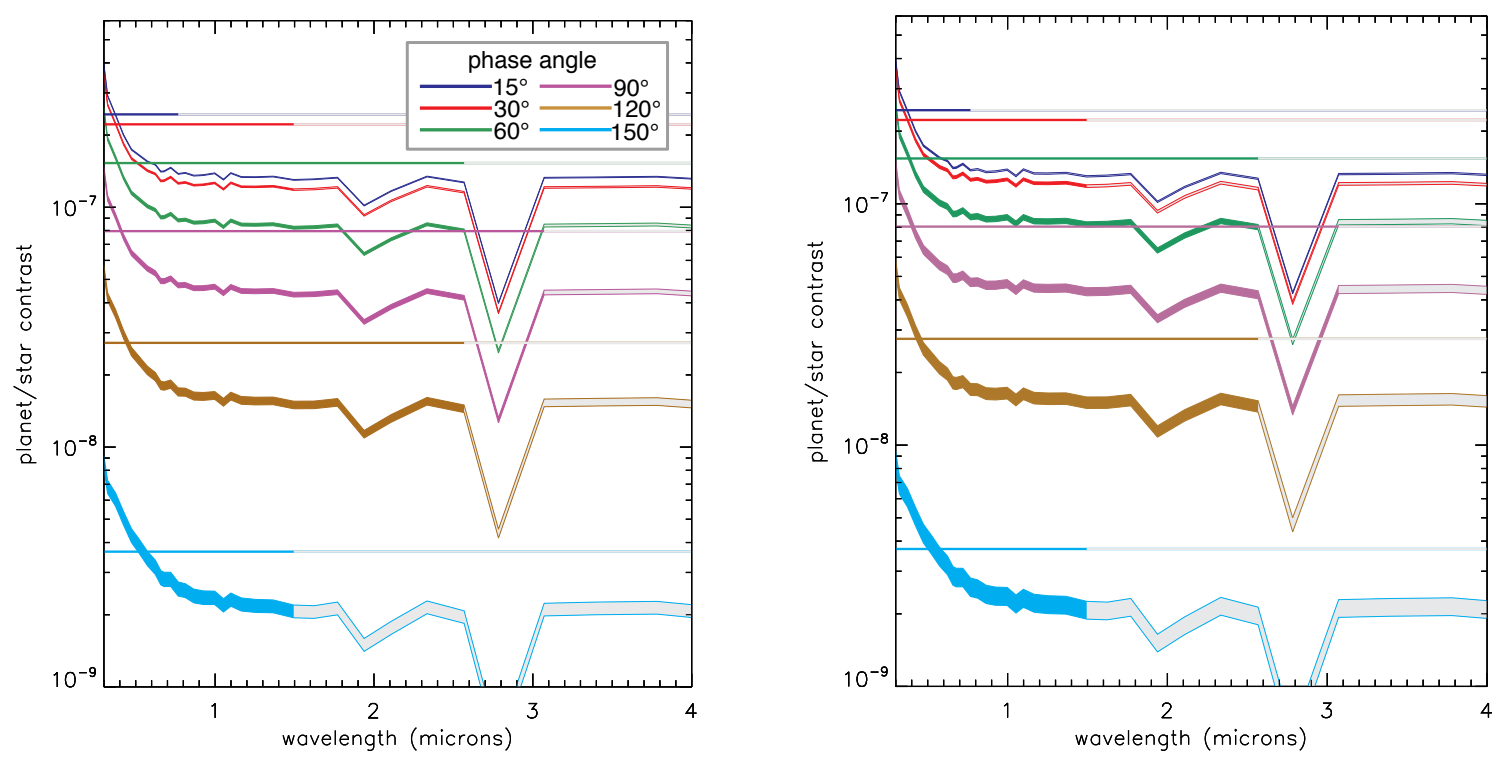

Fig. B.3. Reflection spectra computed for a dry planet with an Earth-like atmosphere in synchronous rotation (left) and 3:2 spin-orbit resonance (right).
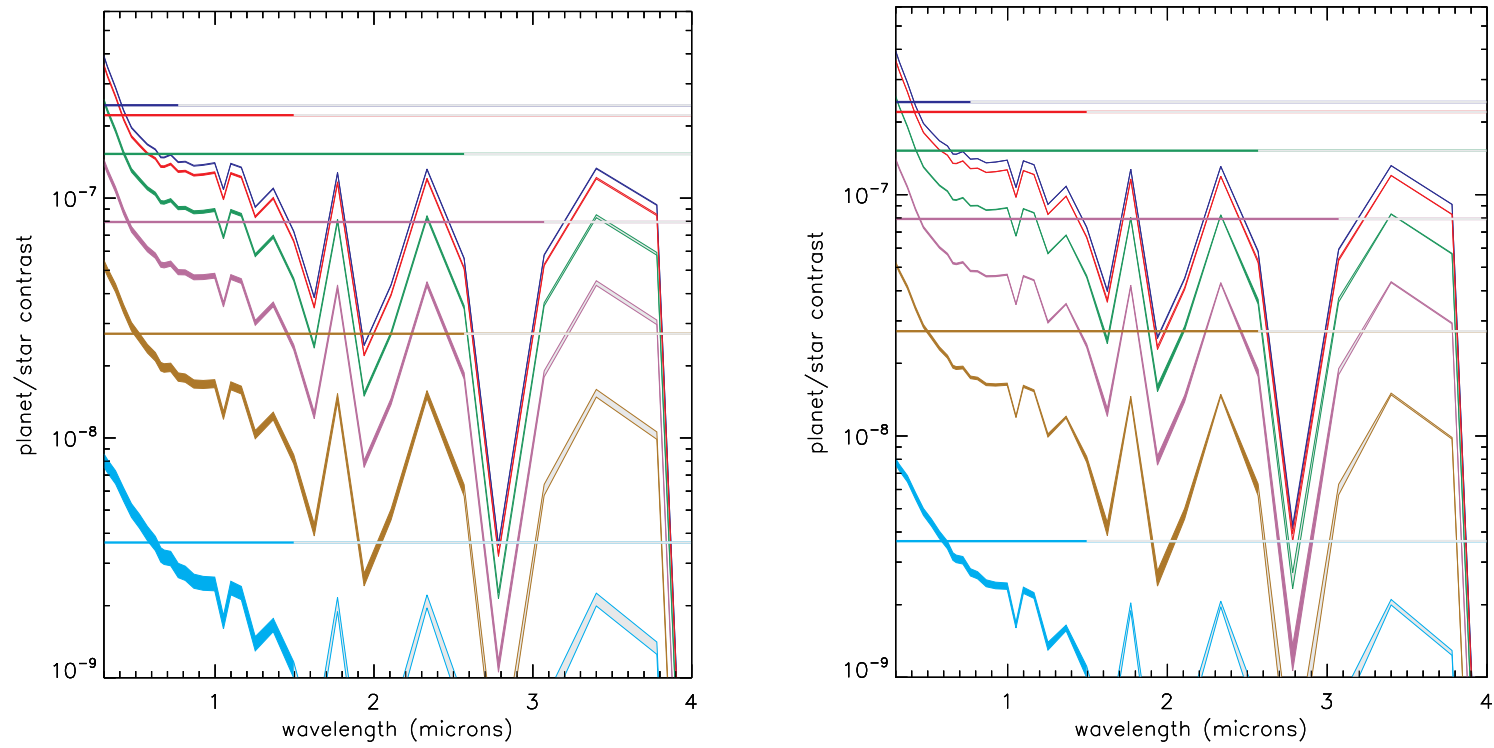

Fig. B.4. Reflection spectra computed for a dry planet with a 1 bar $\mathrm{CO}_{2}$-dominated atmosphere in synchronous rotation (left) and 3:2 spin-orbit resonance $($ right $)$.

- Aquaplanet, mainly frozen, 1 bar of $\mathrm{N}_{2} ; 376 \mathrm{ppm}$ of $\mathrm{CO}_{2}$ (Fig. B.18): the spectra are similar to a present Earth spectrum, without the $\mathrm{O}_{3}$ band and with shallower water vapor absorption due to lower temperatures.

- Aquaplanet, 1 bar of $\mathrm{CO}_{2}$ (Fig. B.19): there are no difference between the synchronous and 3:2 cases. All atmospheric windows are closed by either $\mathrm{CO}_{2}$ or $\mathrm{H}_{2} \mathrm{O}$ absorption and the emerging spectrum come from different but horizontally uniform layers.

\section{B.4. Thermal phase curves}

Emission light curves are controlled by the temperature longitudinal distribution at the surface if the atmosphere is transparent in the observed band or at the emitting atmospheric layer otherwise.
- Dry case, Earth-like atmosphere (Fig. B.20): in both cases, the $15 \mu \mathrm{m} \mathrm{CO}$ band is emitted from a uniform high altitude layer, which produces a flat phase curve. In the synchronous case, other bands exhibit phase curves that are similar to those of an airless planet except at high phase angle and long wavelengths, where the warming of the nightside by atmospheric circulation flattens the curves. In the 3:2 case, the flattening is more pronounced and is associated with a lag as surface temperature peaks in the afternoon.

- Dry case, 1 bar of $\mathrm{CO}_{2}$ (Fig. B.21): similar to the dry Earth case except that the $6.7 \mu \mathrm{m}$ band is flattened due $\mathrm{CO}_{2} \mathrm{CIA}$ absorption.

- Aquaplanet, mainly frozen, 10 mbar of $\mathrm{N}_{2} ; 376 \mathrm{ppm}$ of $\mathrm{CO}_{2}$ (Fig. B.22): here, the $15 \mu \mathrm{m} \mathrm{CO} \mathrm{CO}_{2}$ band is no longer opaque and exhibits the same behavior as the other bands. Compared with the previous case, and despite a more tenuous 

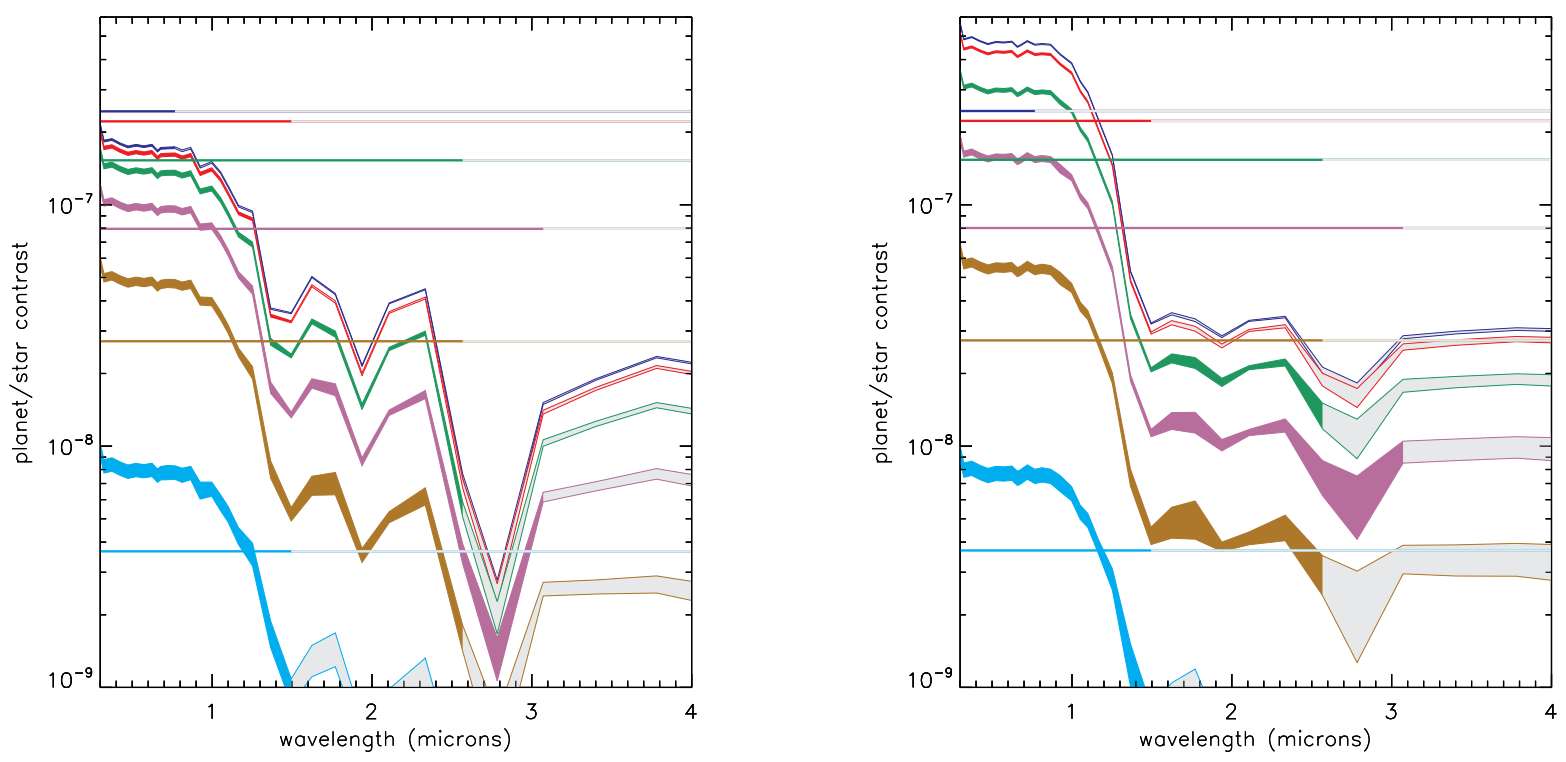

Fig. B.5. Reflection spectra computed for an aquaplanet with a $10 \mathrm{mbar} \mathrm{N}_{2}$-dominated $\left(+376 \mathrm{ppm}\right.$ of $\left.\mathrm{CO}_{2}\right)$ atmosphere in synchronous rotation (left) and 3:2 spin-orbit resonance (right).
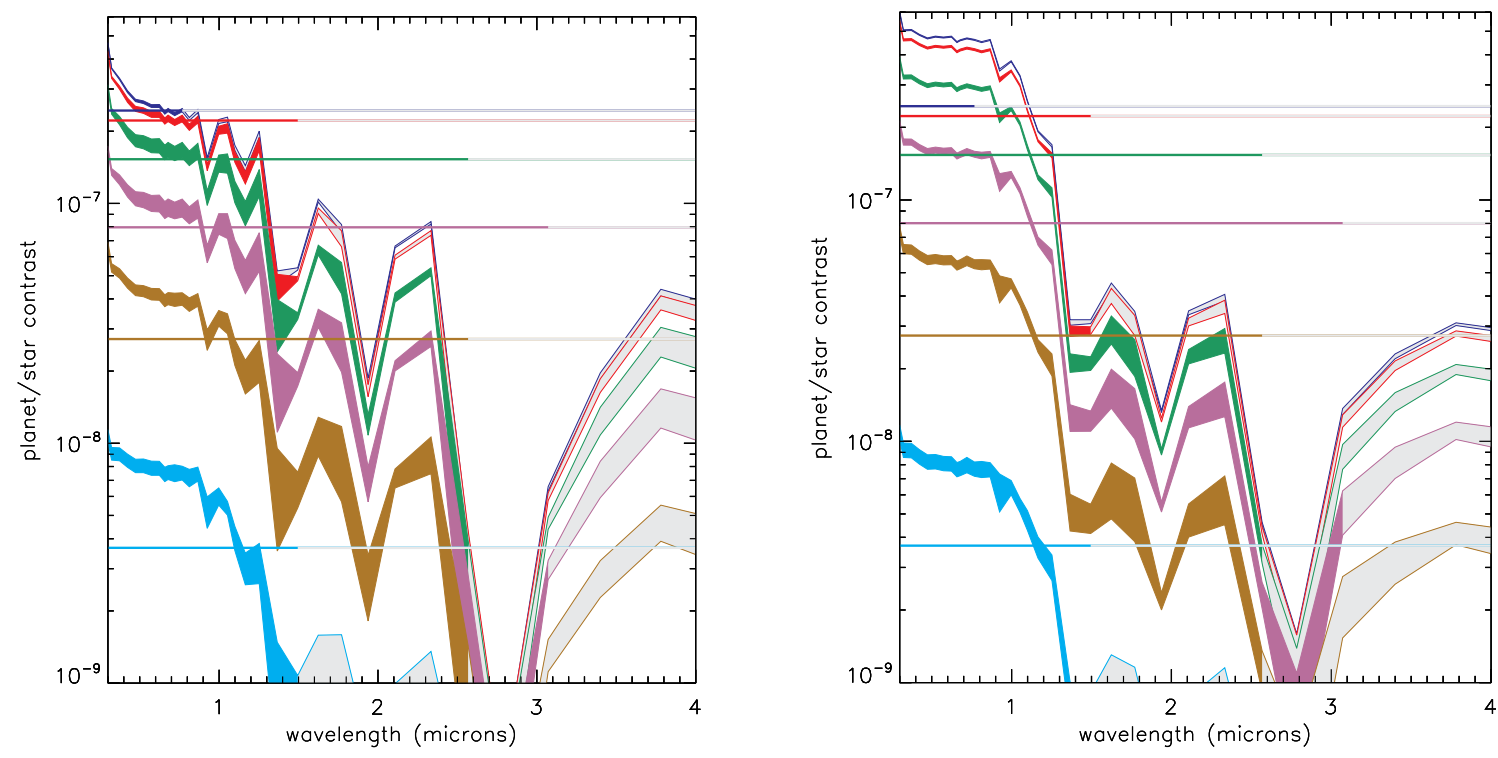

Fig. B.6. Reflection spectra computed for an aquaplanet with an Earth-like atmosphere in synchronous rotation (left) and 3:2 spin-orbit resonance (right).

atmosphere, phase curves are more flattened because of the additional redistribution of latent heat (and the relatively high surface thermal inertia for the asynchronous case).

- Aquaplanet, mainly frozen, 1 bar of $\mathrm{N}_{2} ; 376 \mathrm{ppm}$ of $\mathrm{CO}_{2}$ (Fig. B.23): efficient zonal heat redistribution flattens all the phase curves in the 3:2 case. In the synchronous case, the 11 and $7.7 \mu \mathrm{m}$ bands coming from either surface or clouds show an increase at small phase angle with some asymmetry owing to the patch of clouds eastward of the substellar point (see Fig. B.1). The $6.7 \mu \mathrm{m}$ band emitted in the troposphere is flat with a slight decrease due to the clouds as well. The 15 and $24 \mu \mathrm{m}$ bands are flat and not affected by clouds, emerging from higher levels.

- Aquaplanet, 1 bar of $\mathrm{CO}_{2}$ (Fig. B.24): there are no difference between the synchronous and 3:2 cases. All atmospheric windows are closed by either $\mathrm{CO}_{2}$ or $\mathrm{H}_{2} \mathrm{O}$ absorption and the emission comes from different but horizontally uniform layers. This produces flat light curves at different brightness temperatures with some shallow attenuation by the clouds at the most transparent wavelengths (11 and $7.7 \mu \mathrm{m})$. 
M. Turbet et al.: The habitability of Proxima Centauri b. II.
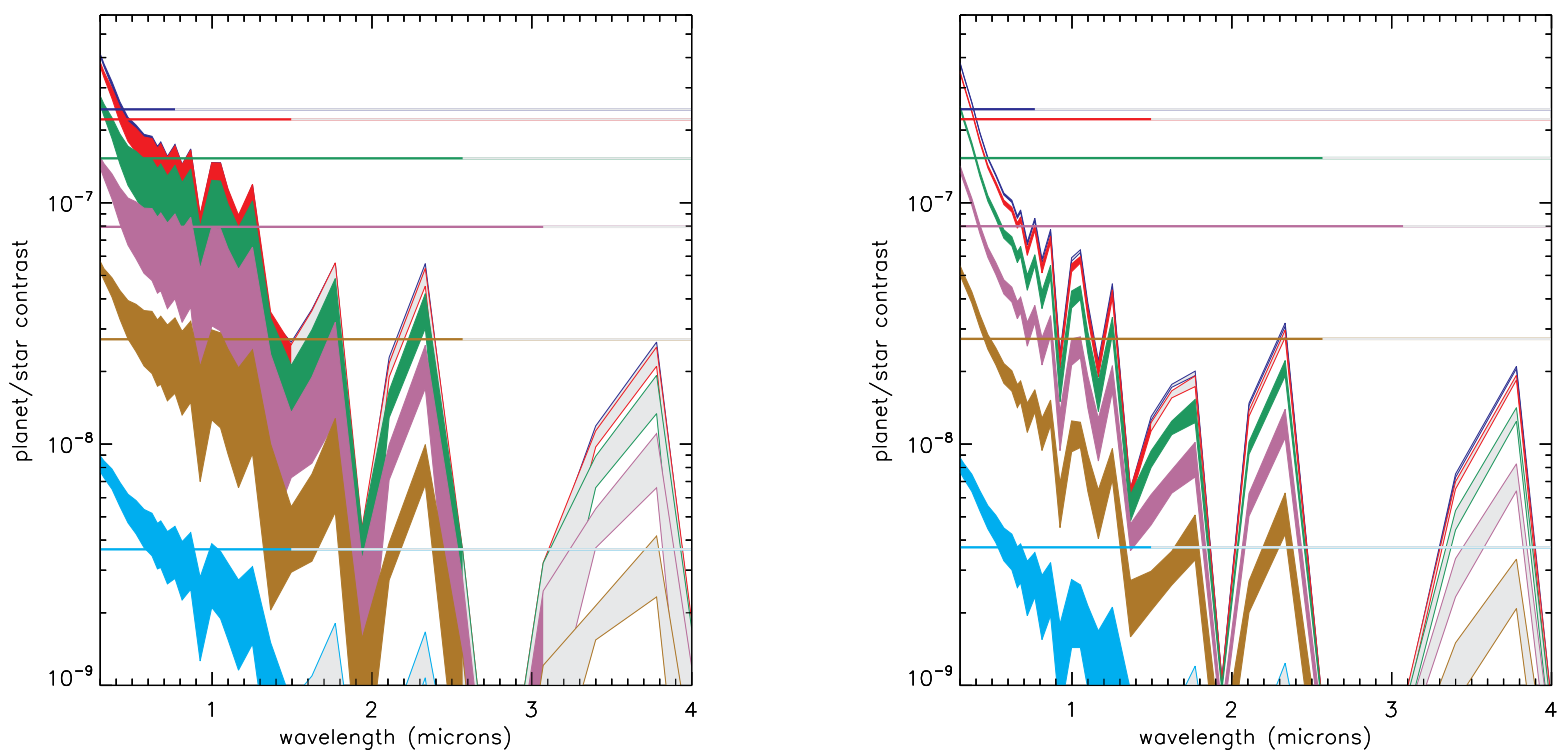

Fig. B.7. Reflection spectra computed for an aquaplanet with a 1 bar $\mathrm{CO}_{2}$-dominated atmosphere in synchronous rotation (left) and 3:2 spin-orbit resonance (right).

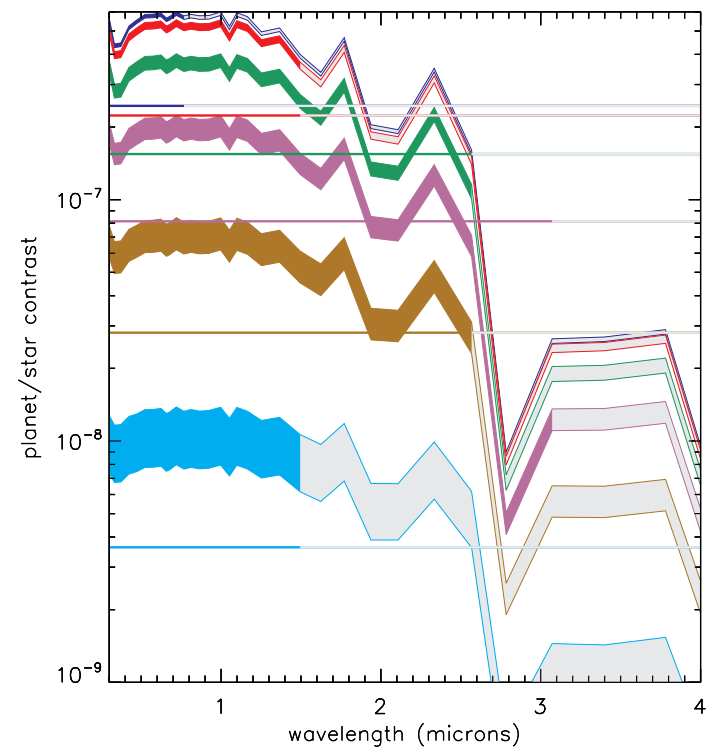

Fig. B.8. Reflection spectra computed for a dry planet with a Venus-like atmosphere (including aerosols). 
A\&A 596, A112 (2016)
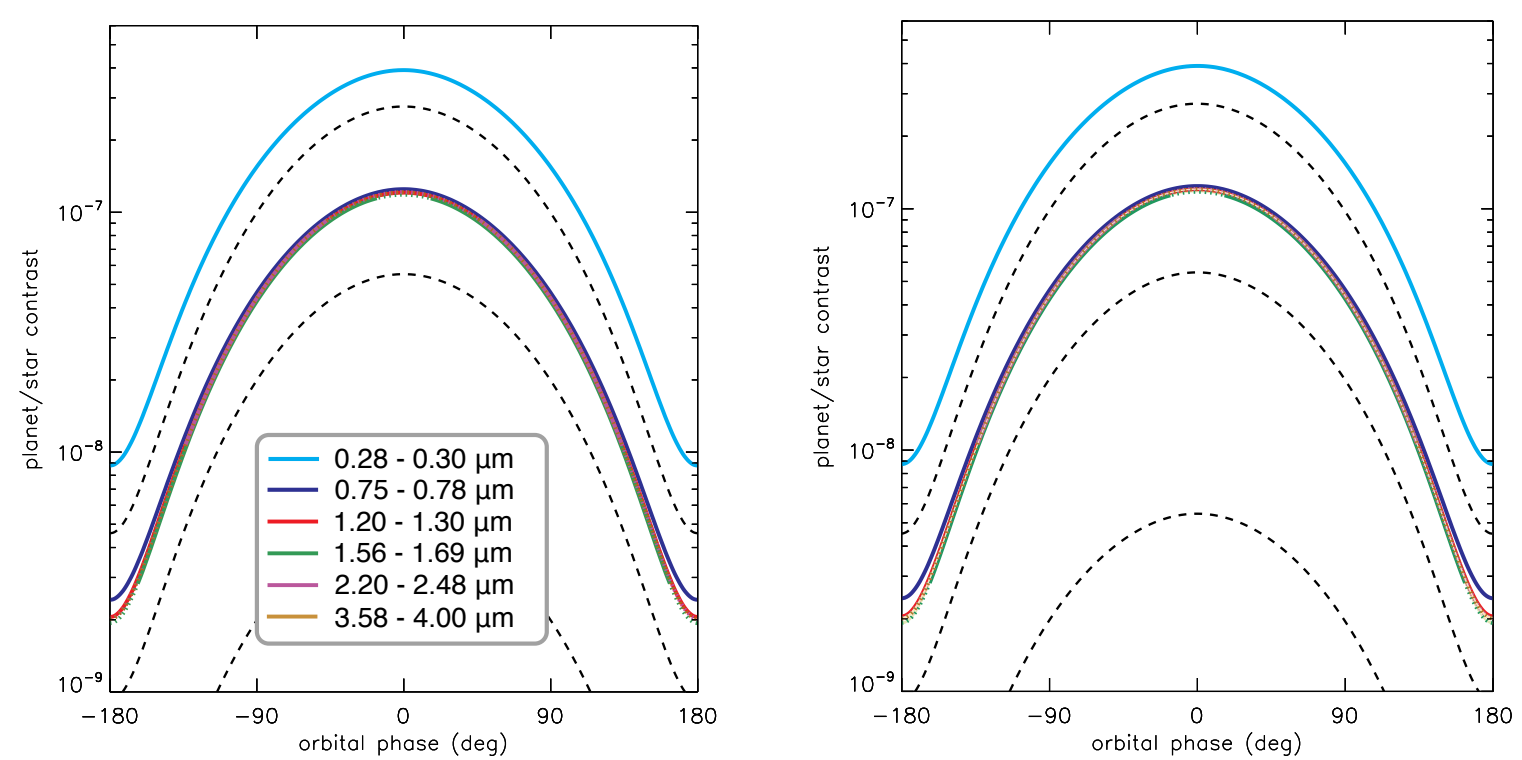

Fig. B.9. Reflection phase curves computed for a dry planet with an Earth-like atmosphere in synchronous rotation (left) and 3:2 spin-orbit resonance (right).
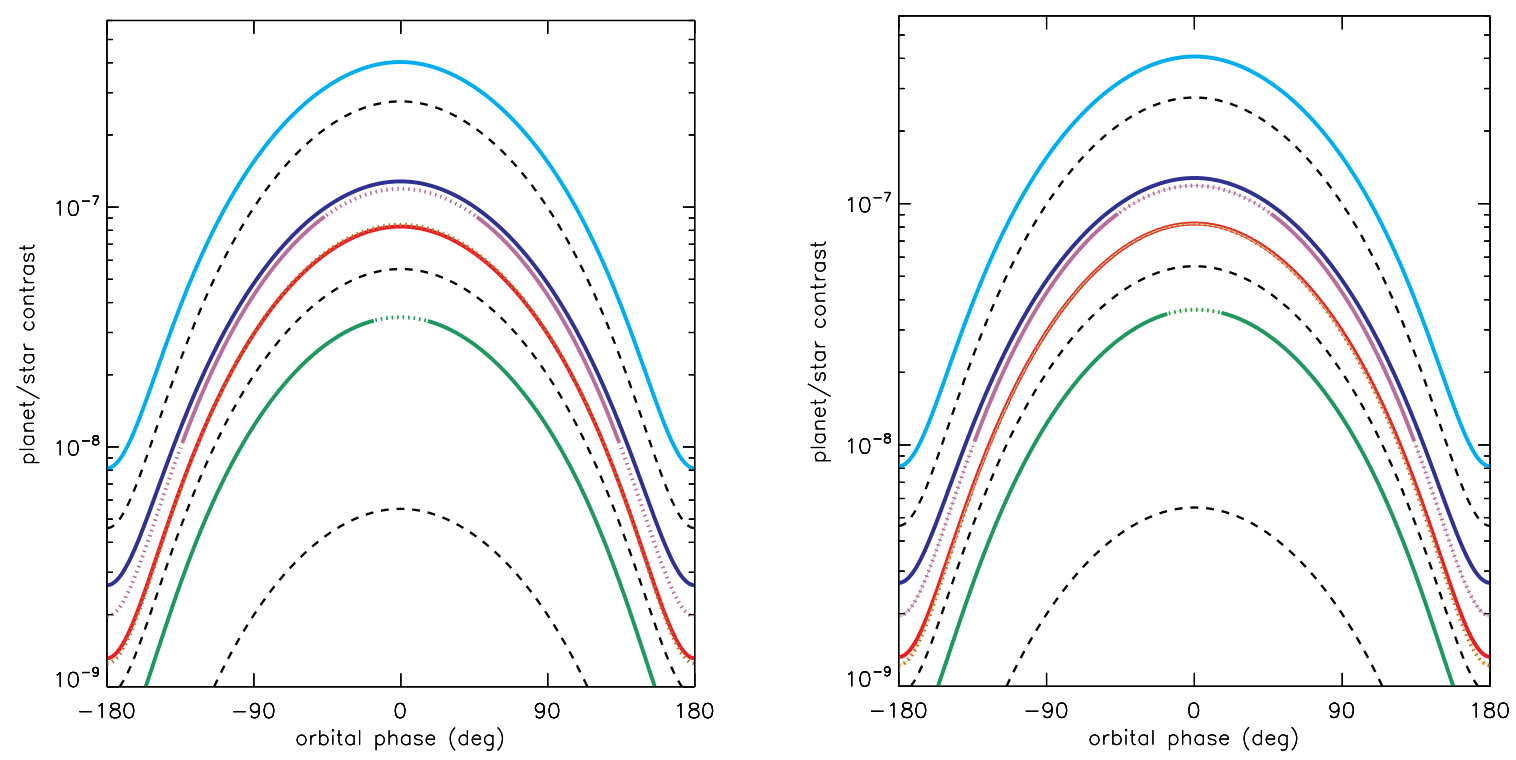

Fig. B.10. Reflection phase curves computed for a dry planet with a 1 bar $\mathrm{CO}_{2}$-dominated atmosphere in synchronous rotation (left) and 3:2 spin-orbit resonance (right). 
M. Turbet et al.: The habitability of Proxima Centauri b. II.
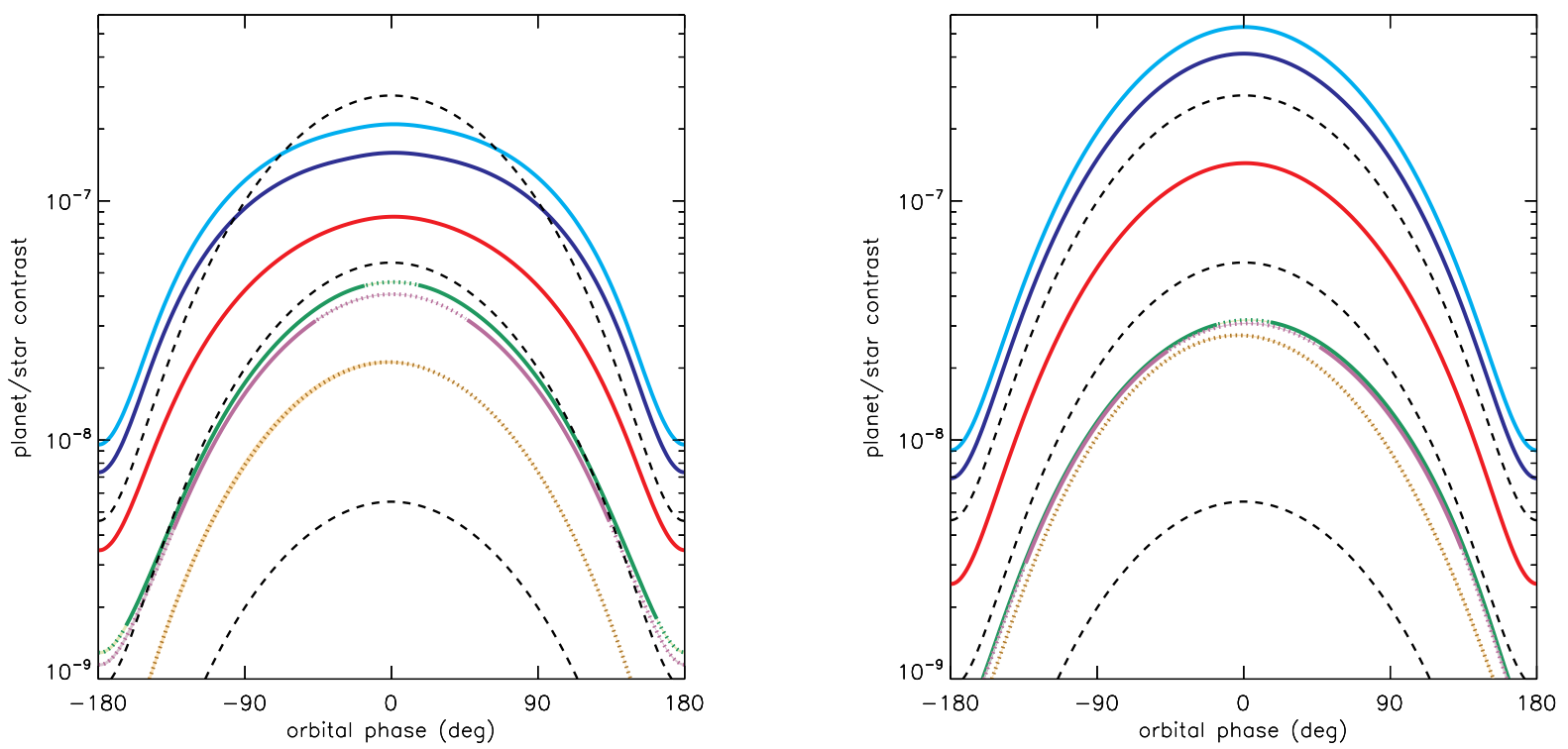

Fig. B.11. Reflection phase curves computed for an aquaplanet with a 10 mbar $\mathrm{N}_{2}$-dominated $\left(+376\right.$ ppm of $\left.\mathrm{CO}_{2}\right)$ atmosphere in synchronous rotation (left) and 3:2 spin-orbit resonance (right).
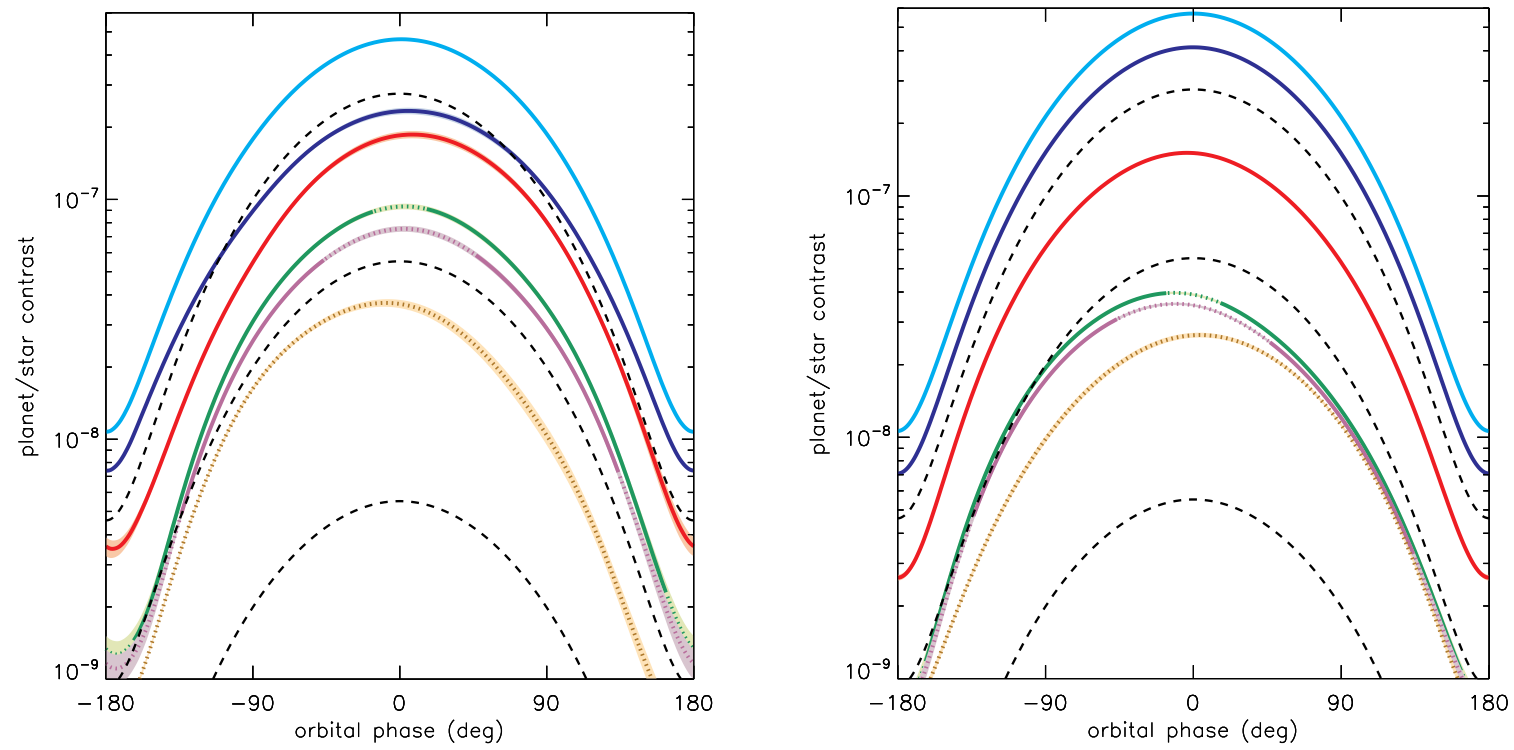

Fig. B.12. Reflection phase curves computed for an aquaplanet with an Earth-like atmosphere in synchronous rotation (left) and 3:2 spin-orbit resonance (right). 

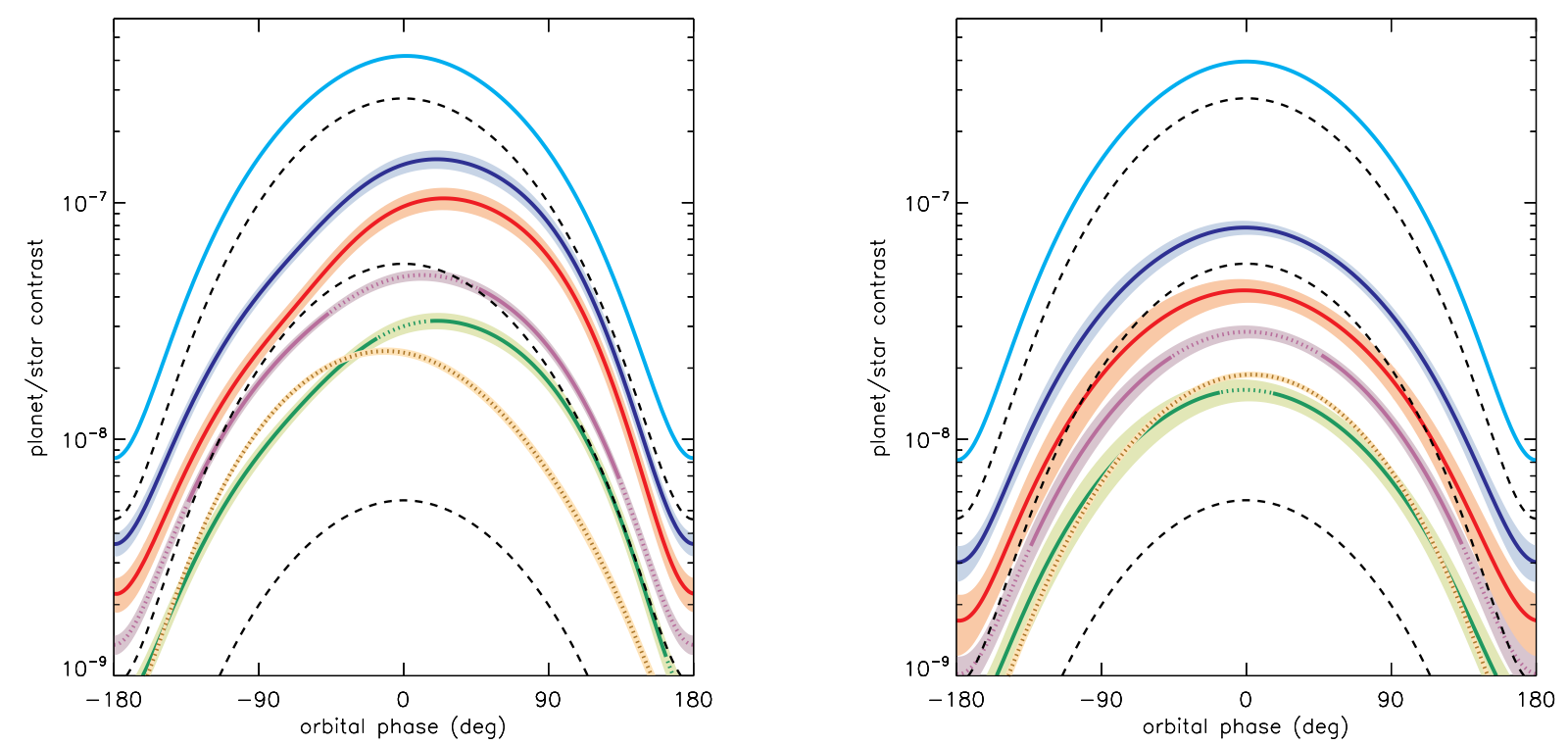

Fig. B.13. Reflection phase curves computed for an aquaplanet with a 1 bar $\mathrm{CO}_{2}$-dominated atmosphere in synchronous rotation (left) and 3:2 spin-orbit resonance (right).

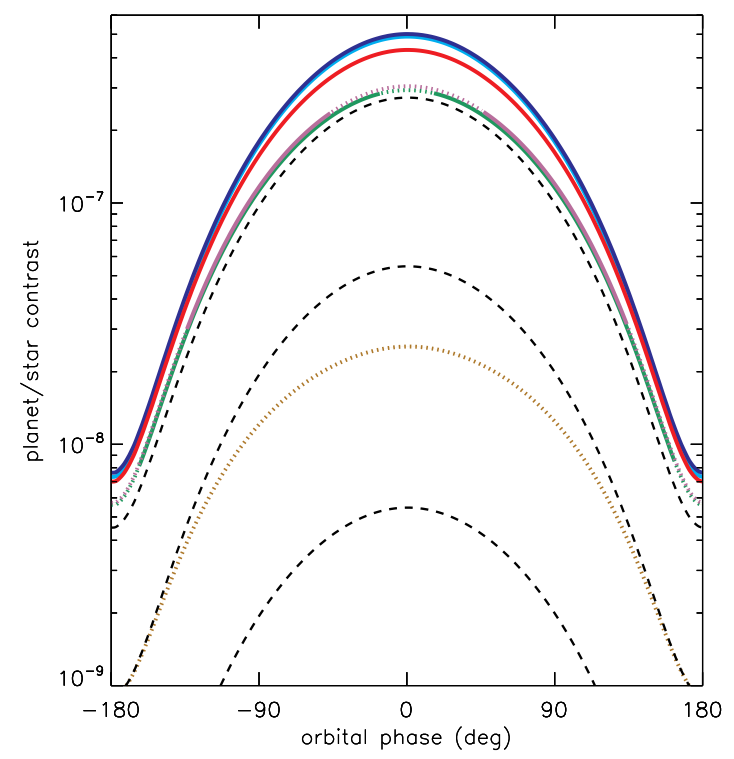

Fig. B.14. Reflection phase curves computed for a dry planet with a Venus-like atmosphere (including aerosols). 
M. Turbet et al.: The habitability of Proxima Centauri b. II.
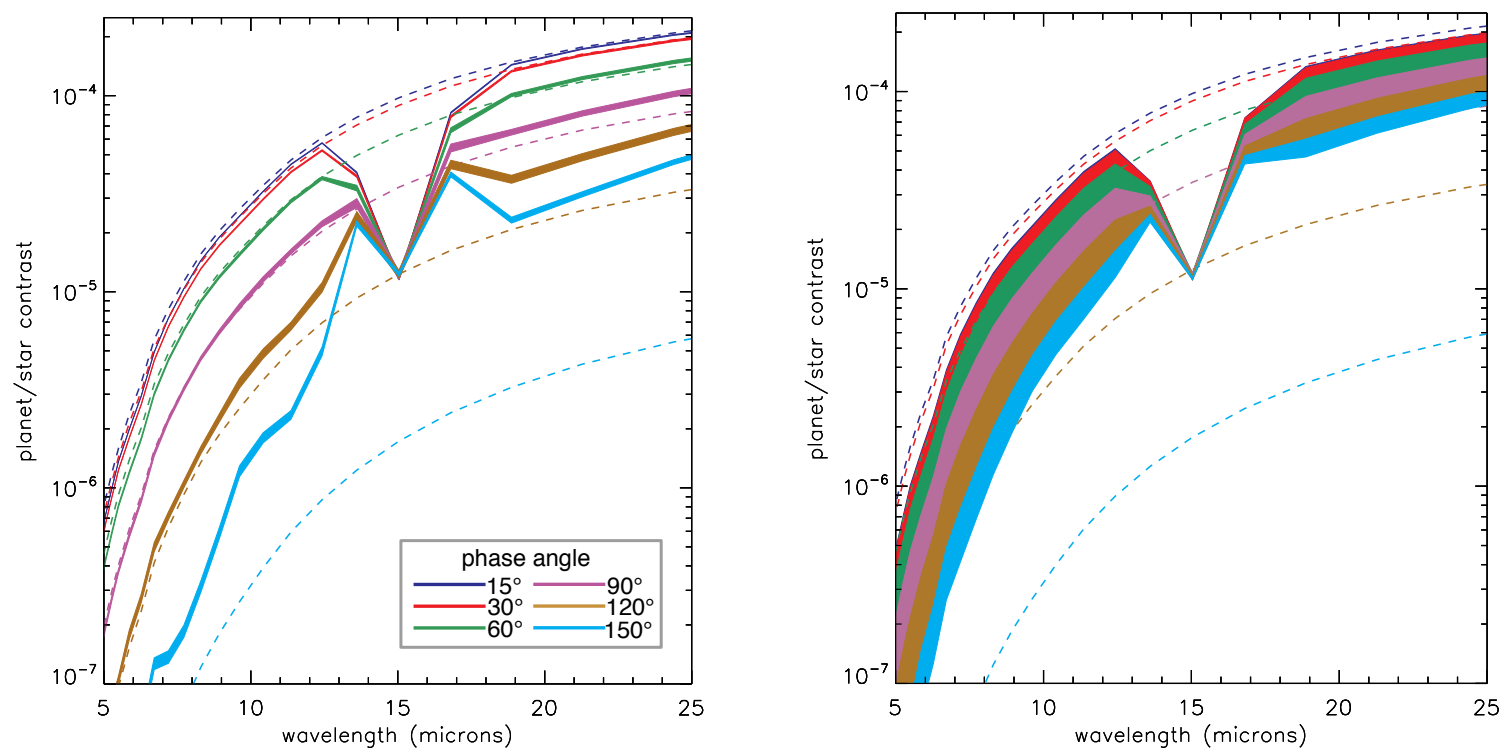

Fig. B.15. Emission spectra computed for a dry planet with an Earth-like atmosphere in synchronous rotation (left) and 3:2 spin-orbit resonance (right).
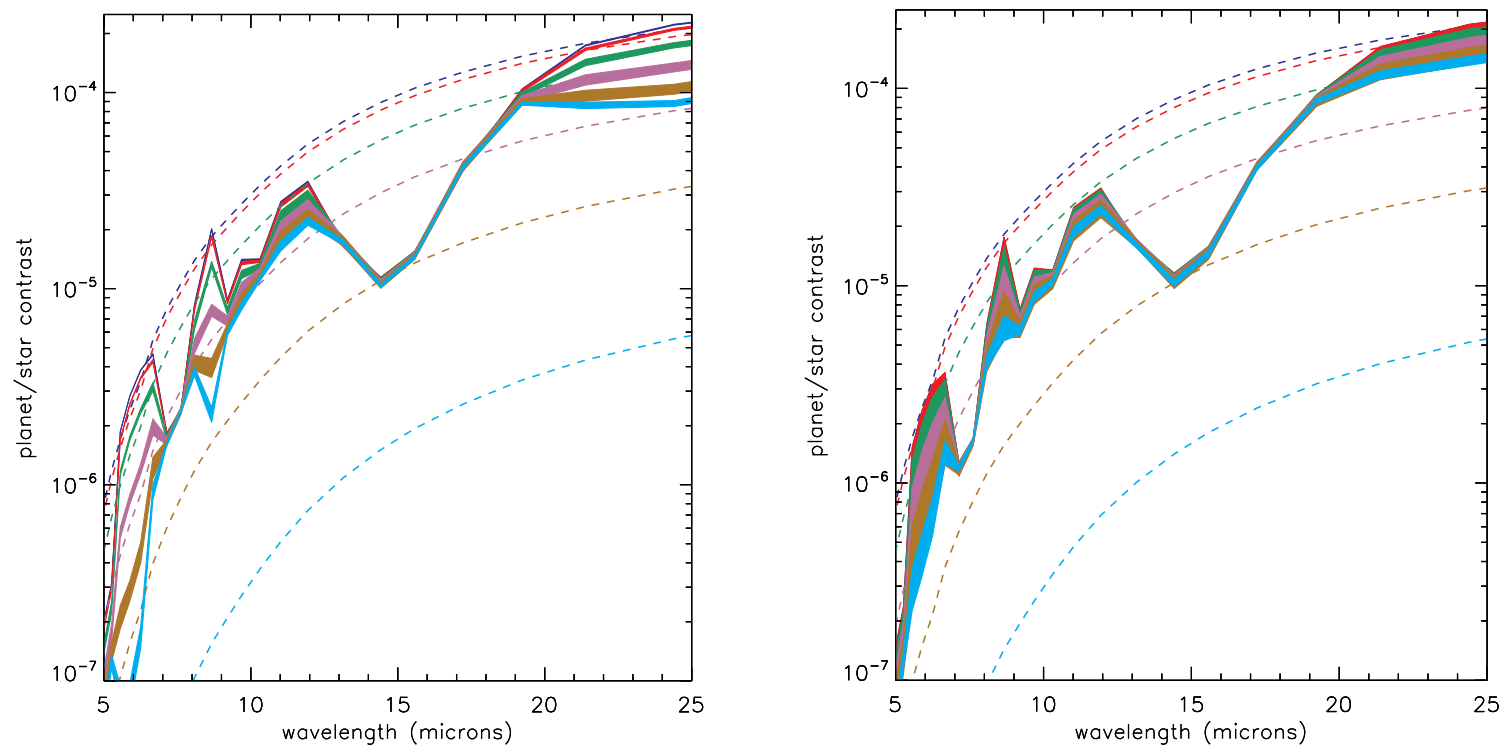

Fig. B.16. Emission spectra computed for a dry planet with a 1 bar $\mathrm{CO}_{2}$-dominated atmosphere in synchronous rotation (left) and 3:2 spin-orbit resonance (right). 
A\&A 596, A112 (2016)
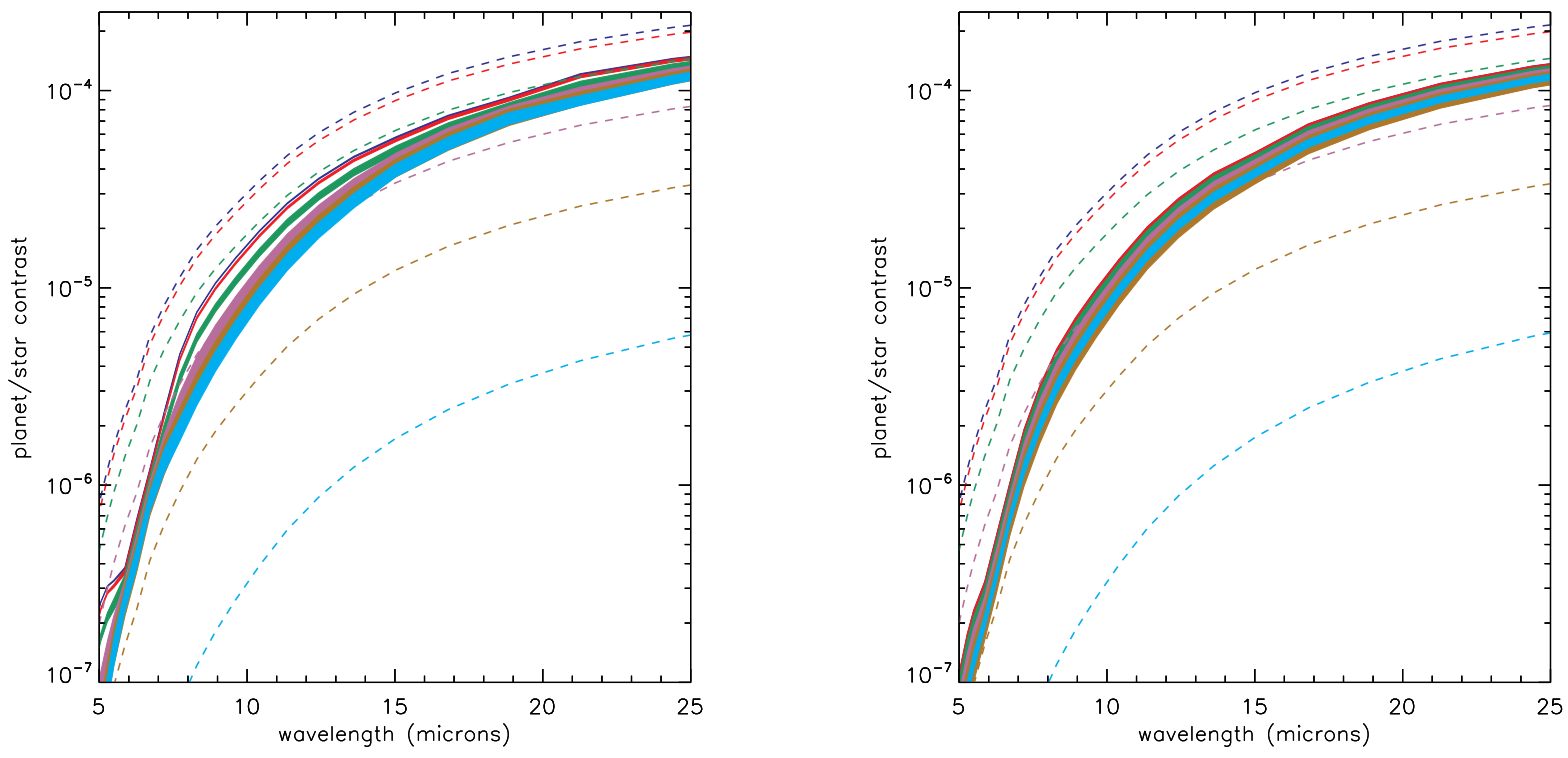

Fig. B.17. Emission spectra computed for an aquaplanet with a $10 \mathrm{mbar} \mathrm{N}_{2}$-dominated $\left(+376 \mathrm{ppm}\right.$ of $\left.\mathrm{CO}_{2}\right)$ atmosphere in synchronous rotation (left) and 3:2 spin-orbit resonance (right).
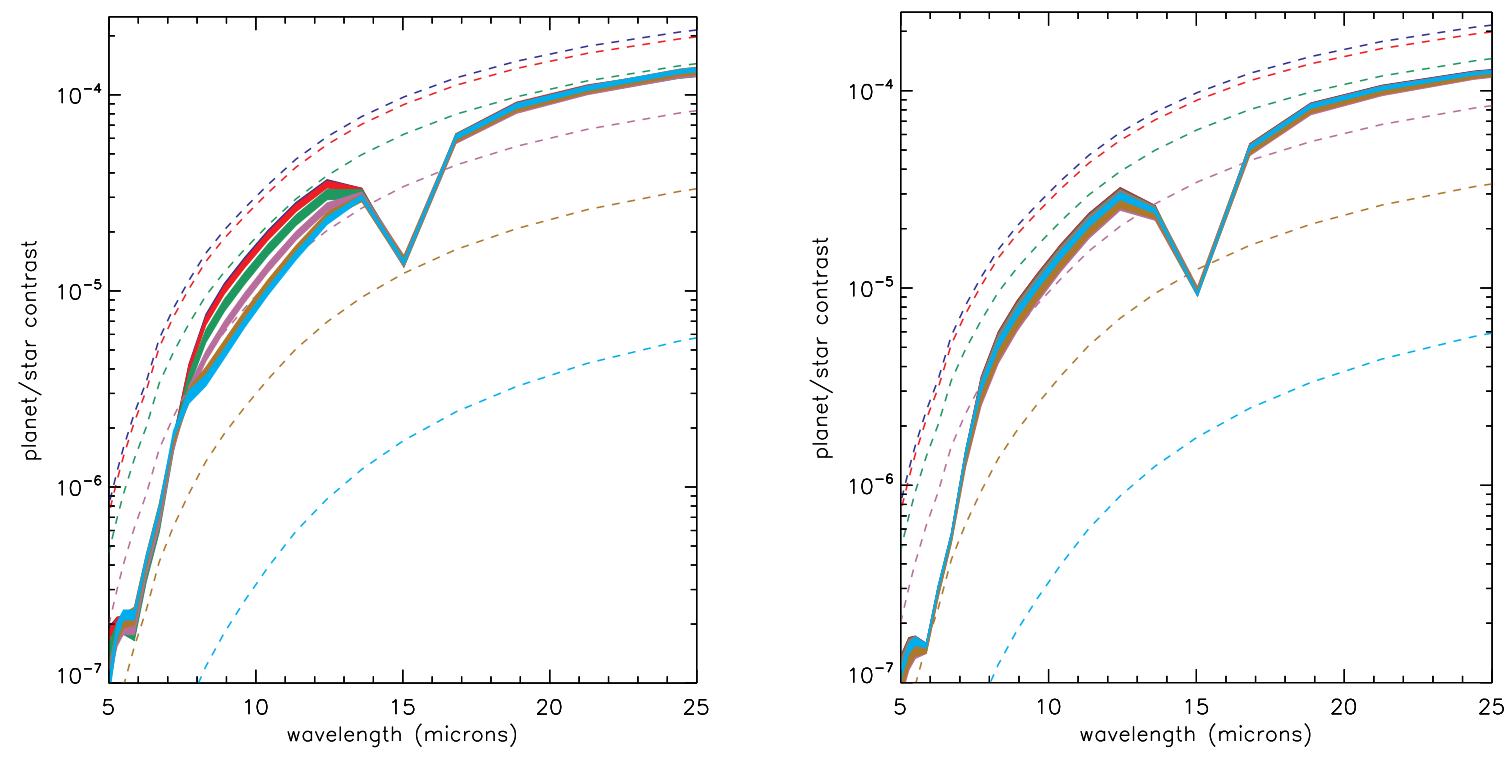

Fig. B.18. Emission spectra computed for an aquaplanet with an Earth-like atmosphere in synchronous rotation (left) and 3:2 spin-orbit resonance (right). 
M. Turbet et al.: The habitability of Proxima Centauri b. II.
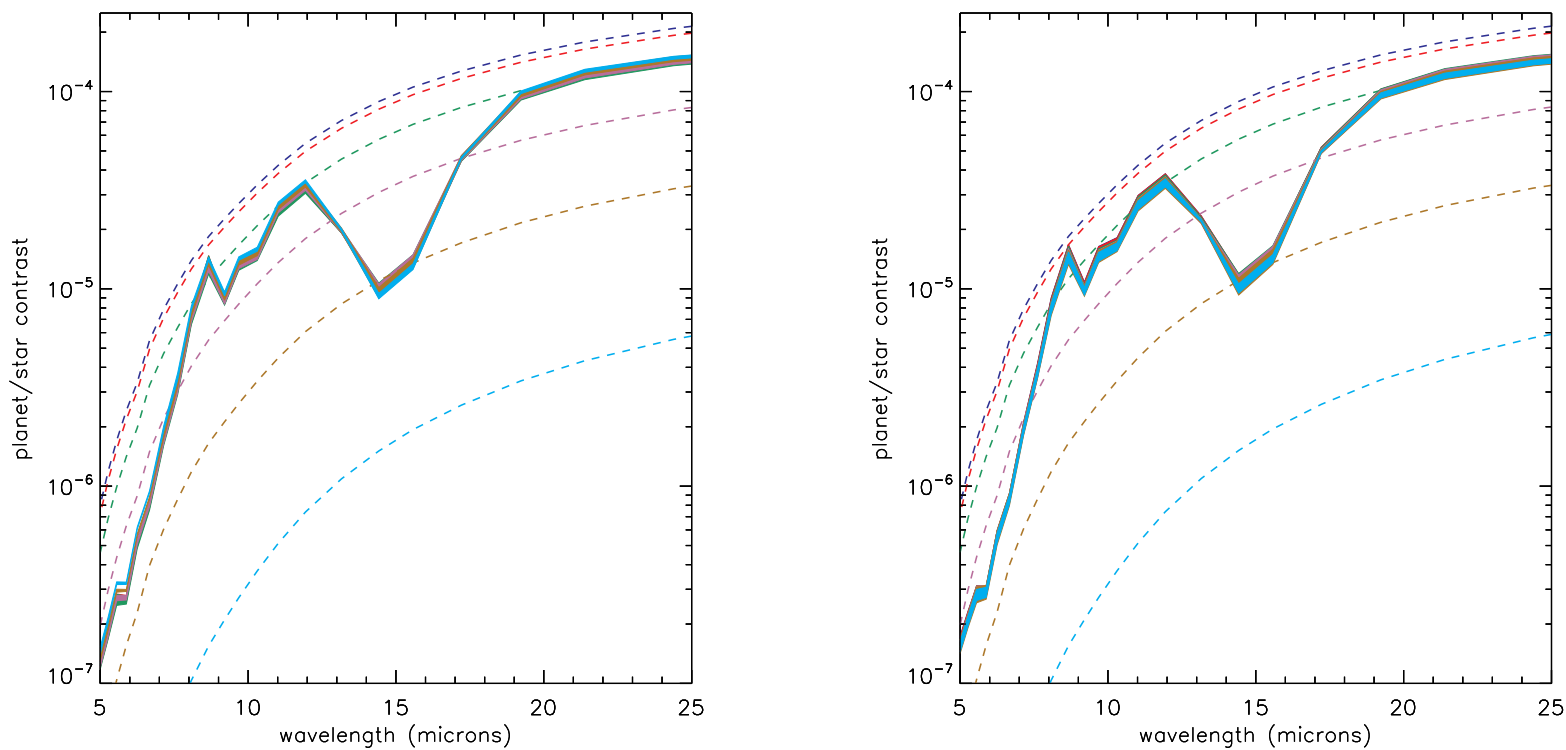

Fig. B.19. Emission spectra computed for an aquaplanet with a 1 bar $\mathrm{CO}_{2}$-dominated atmosphere in synchronous rotation (left) and 3:2 spin-orbit resonance (right).
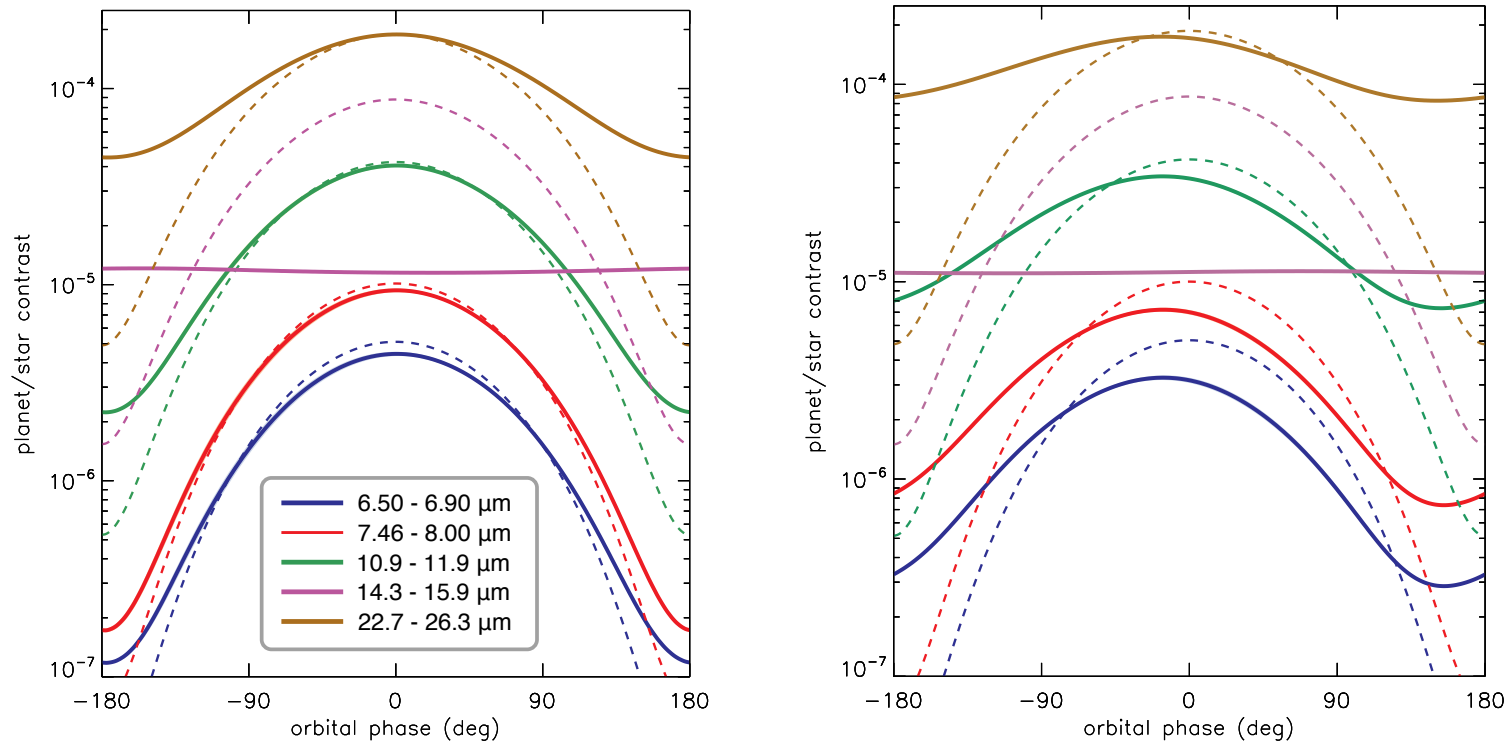

Fig. B.20. Emission phase curves computed for a dry planet with an Earth-like atmosphere in synchronous rotation (left) and 3:2 spin-orbit resonance (right). 
A\&A 596, A112 (2016)
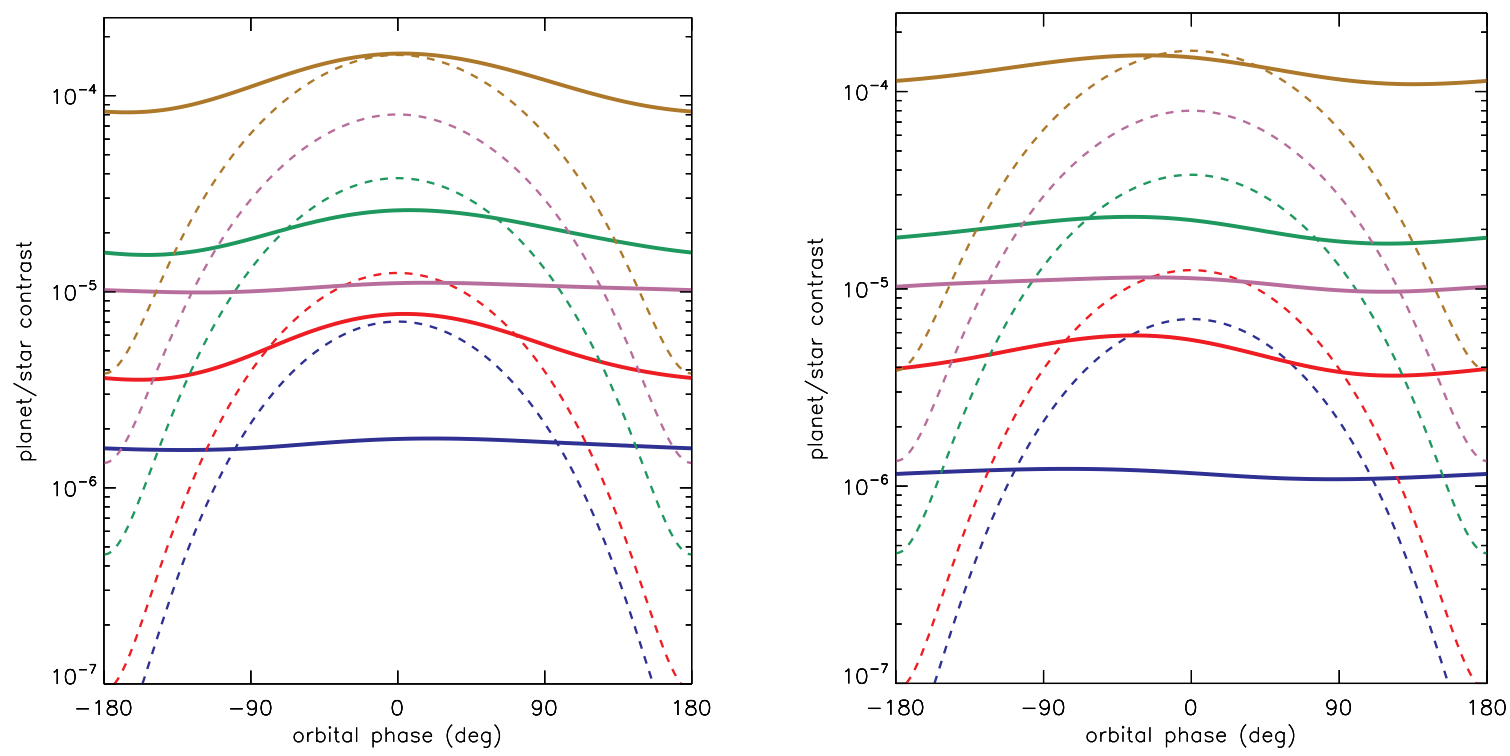

Fig. B.21. Emission phase curves computed for a dry planet with a 1 bar $\mathrm{CO}_{2}$-dominated atmosphere in synchronous rotation (left) and 3:2 spin-orbit resonance (right).
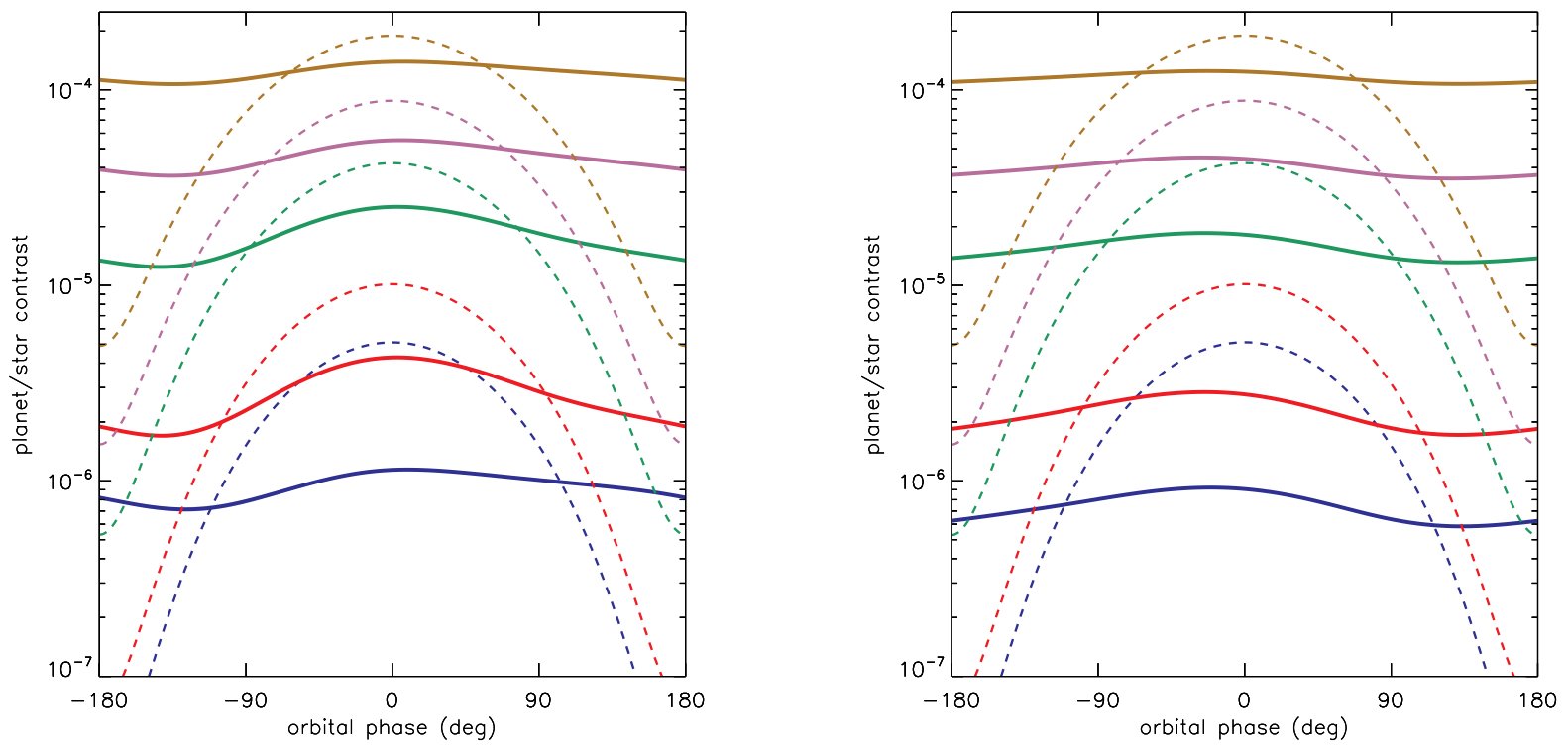

Fig. B.22. Emission phase curves computed for an aquaplanet with a 10 mbar $\mathrm{N}_{2}$-dominated $\left(+376 \mathrm{ppm}\right.$ of $\left.\mathrm{CO}_{2}\right)$ atmosphere in synchronous rotation (left) and 3:2 spin-orbit resonance (right). 
M. Turbet et al.: The habitability of Proxima Centauri b. II.
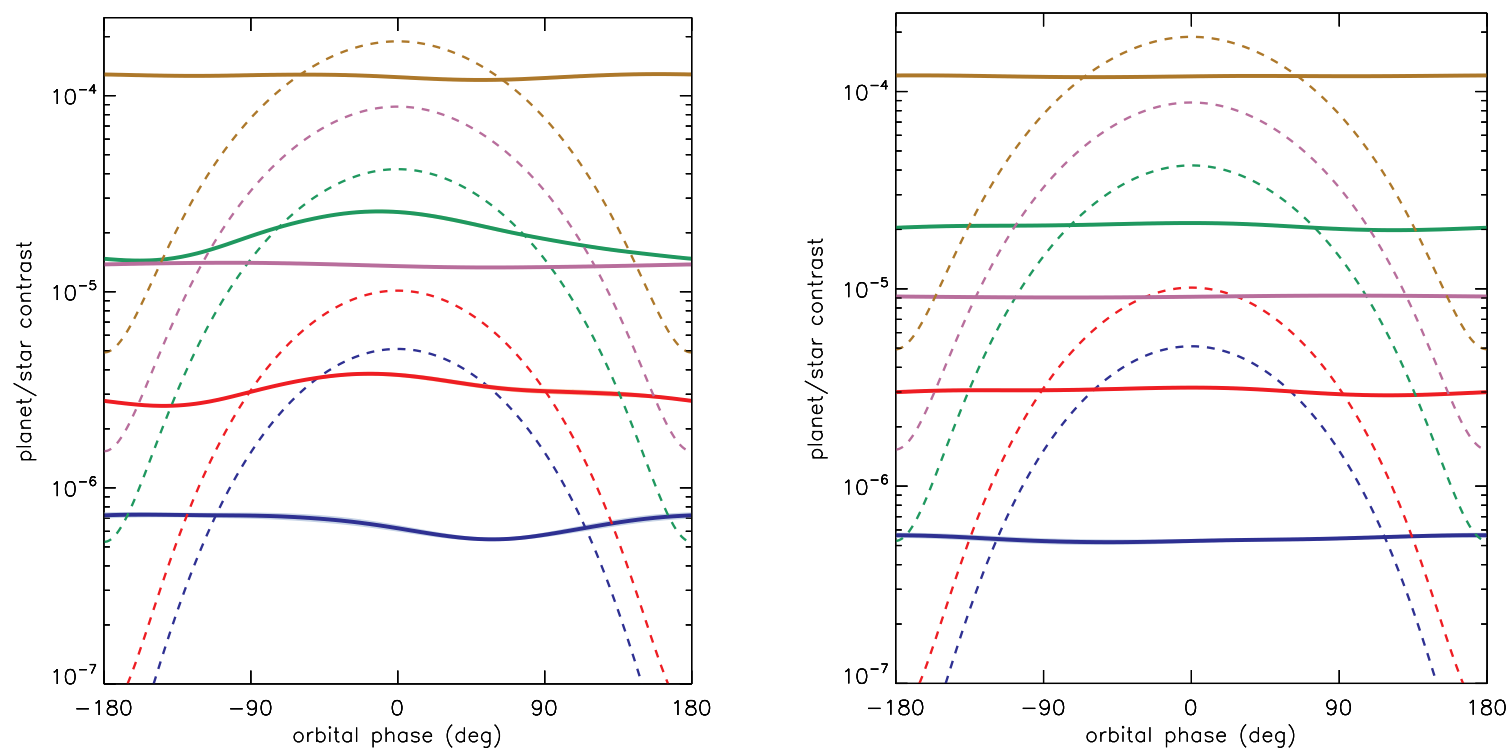

Fig. B.23. Emission phase curves computed for an aquaplanet with an Earth-like atmosphere in synchronous rotation (left) and 3:2 spin-orbit resonance (right).
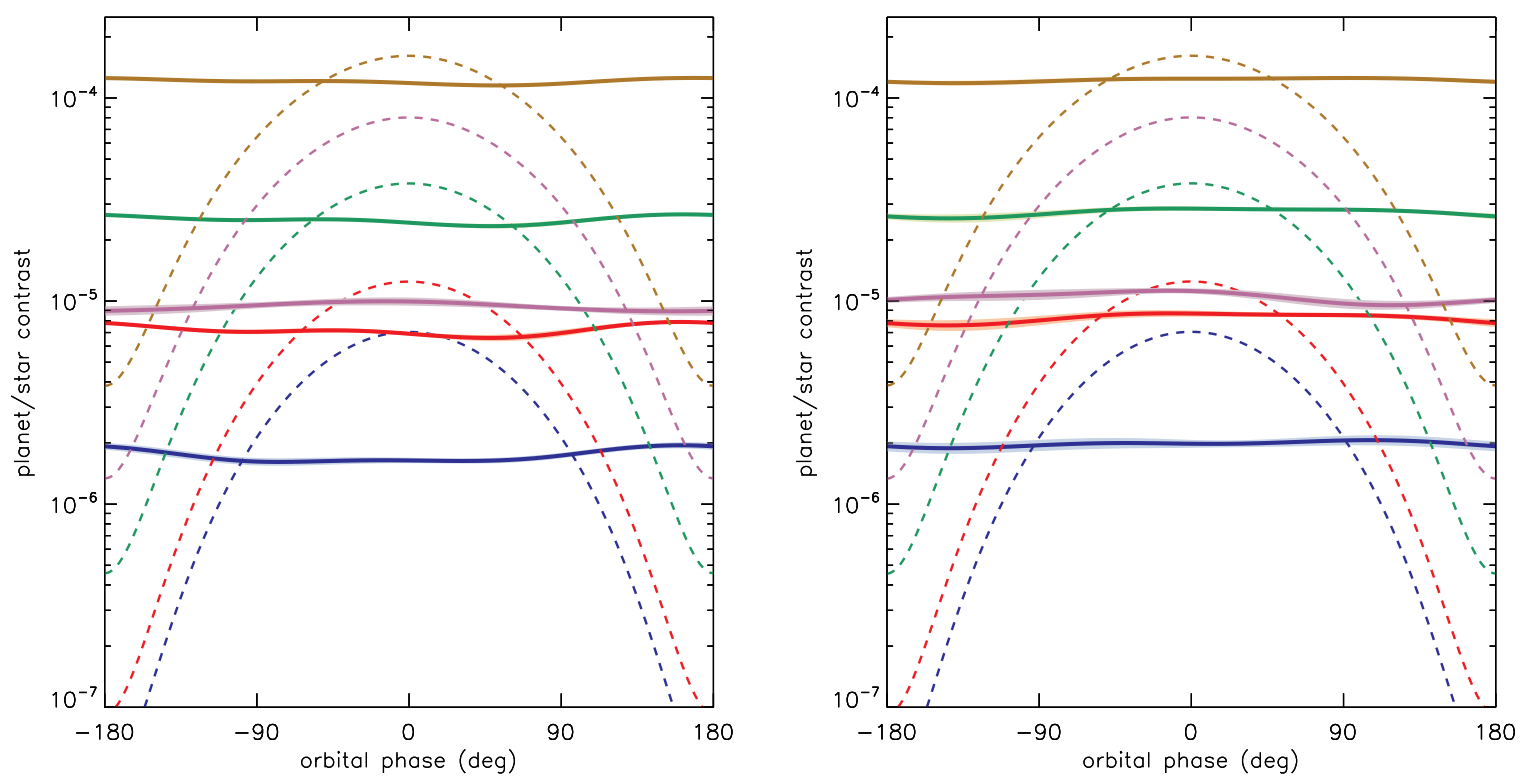

Fig. B.24. Emission phase curves computed for an aquaplanet with a 1 bar $\mathrm{CO}_{2}$-dominated atmosphere in synchronous rotation (left) and 3:2 spin-orbit resonance (right). 